\title{
Enantioselective Synthesis of Six-Membered Palladacycles Having Metal-Bound Stereogenic Carbons: Isolation and Reactivity of Palladacycles Containing Readily Accessible 0 -Hydrogens
}

\author{
Brenda J. Burke and Larry E. Overman*
}

Department of Chemistry, 516 Rowland Hall, University of California, Irvine, CA 92697-2025

\section{Supporting Information}

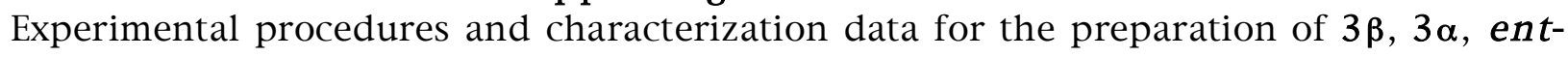

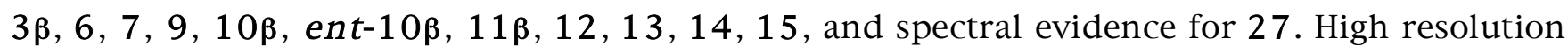
mass spectrometric data for ${ }^{15} \mathrm{~N}$-labelled compounds $17,21,\left[{ }^{15} \mathrm{~N}\right] 8$ and $\left[{ }^{15} \mathrm{~N}\right] 2$ and ${ }^{17} \mathrm{O}-$ labelled compounds $24,\left[{ }^{17} \mathrm{O}\right] 5,25,\left[{ }^{17} \mathrm{O}\right] 22$ and $\left[{ }^{17} \mathrm{O}\right] 2$. Infrared spectra for $3 \square, 13,10 \square, 14$, $\left[{ }^{17} \mathrm{O}\right] 3 \square,\left[{ }^{17} \mathrm{O}\right] 13,\left[{ }^{17} \mathrm{O}\right] 10 \square,\left[{ }^{17} \mathrm{O}\right] 14$. X-ray crystallographic data for compounds $3 \square$ and $10 \square$.

General Methods. All reactions were carried out in oven-dried $\left(180{ }^{\circ} \mathrm{C}\right)$ glassware under a $\mathrm{N}_{2}$ or argon atmosphere. Tetrahydrofuran (THF), diethyl ether $\left(\mathrm{Et}_{2} \mathrm{O}\right)$, dichloromethane $\left(\mathrm{CH}_{2} \mathrm{Cl}_{2}\right)$, toluene (PhMe), dimethoxyethane (DME), hexanes, diisopropylethylamine (DIEA), benzene $(\mathrm{PhH})$, dimethylformamide (DMF), pyridine (pyr), acetonitrile (MeCN) methanol $(\mathrm{MeOH})$, and triethylamine $\left(\mathrm{Et}_{3} \mathrm{~N}\right)$ were degassed with argon and then passed through appropriate column(s) made by Glass Contours, Inc. to remove water and/or oxygen. ${ }^{1}$ Dimethylacetamide (DMA) was distilled from $\mathrm{CaH}_{2}$ under reduced pressure and stored in a sealed flask under argon. Other reagents and solvents were used as received from the manufacturer unless otherwise indicated. Indicated molarities of organolithium reagents were established by titration with menthol/fluorine. ${ }^{2}$ Concentrations were performed under reduced pressure (ca. $15 \mathrm{~mm} \mathrm{Hg}$ ) using a Buchi rotary evaporator. Thin Layer Chromatography (TLC) was performed using EM Science Silica Gel $60 \mathrm{PF}_{254}$. Flash Chromatography was performed using Merck silica gel (0.040-0.063 mesh) unless otherwise indicated.

\footnotetext{
${ }^{1}$ Pangborn, A. B.; Giardello, M. A.; Grubbs, R. H.; Rosen, R. K.; Timmers, F. J. Organometallics, 1996, 15, 1518-1520.

2 Posner, G. H.; Lentz, C. M. J. Am. Chem. Soc. 1979, 101, 934-946.
} 
Instrumentation. ${ }^{1} \mathrm{H},{ }^{13} \mathrm{C},{ }^{31} \mathrm{P}$ and ${ }^{15} \mathrm{~N}$ NMR spectra were measured at 500, 125, 162 and $51 \mathrm{MHz}$, respectively, with a Bruker Avance 500 FT NMR spectrometer. Chemical shifts are reported as $\square$ values in ppm. Coupling constants are reported in $\mathrm{Hz}$ and refer to apparent multiplicities and not true coupling constants. Multiplicity is indicated as follows: s (singlet); $d$ (doublet); t (triplet); q (quartet); m (multiplet); app d (apparent doublet); app t (apparent triplet); dd (doublet of doublets); ddd (doublet, doublet of doublets); dddd (doublet, doublet, doublet of doublets); br s (broad singlet). Infrared spectra (IR) were obtained on an ASI Applied Systems ReactIR 1000 and are reported in wave numbers $\left(\mathrm{cm}^{-1}\right)$. Optical rotations were measured with a JASCO DP-1010 halogen lamp polarimeter; concentration $c$ is reported in $\mathrm{g} / 100 \mathrm{~mL}$. Mass spectra and high-resolution mass spectra (HRMS) were obtained on a MicroMass Analytical 7070E (CI) spectrometer, a MicroMass AutoSpec E (FAB) spectrometer, or a MicroMass LCT (ES) spectrometer. Microanalyses were performed by Atlantic Microlabs, Atlanta, GA.

General Procedure for the Asymmetric Palladacyclization of Aryl Triflates (GP1). A base-washed, oven-dried sealed tube equipped with a magnetic stirring bar and a Teflon ${ }^{\circledR}$ seal was charged with the appropriate aryl triflate ( 1 equiv), $\operatorname{Pd}(\mathrm{OAc})_{2}(1$ equiv) and the appropriate chiral bisphosphine (2 equiv). Deoxygenated $N, N$-dimethylacetamide (DMA, distilled from $\mathrm{CaH}_{2}$ into a sealed tube under reduced pressure, backfilled with Ar, then sparged with Ar for $2 \mathrm{~h}, 0.1 \mathrm{M}$ ) and 1,2,2,6,6-pentamethylpiperidine (PMP, distilled from $\mathrm{CaH}_{2}$ under into a sealed tube under reduced pressure, 4 equiv) were added to the solids. A stream of Ar was bubbled through the resulting mixture for $15 \mathrm{~min}$ and then the sealed tube was flushed with a steady stream of Ar for an additional 15 min. The Teflon ${ }^{\circledR}$ seal was tightened and the solution was maintained at $70-80{ }^{\circ} \mathrm{C}$ until the aryl triflate was no longer detected by TLC analysis (typically 10-16 h). The solution was allowed to cool to room temperature, diluted with EtOAc and poured into saturated aqueous $\mathrm{NaHCO}_{3}$. The aqueous phase was separated and extracted with EtOAc. The combined organic extracts were dried $\left(\mathrm{Na}_{2} \mathrm{SO}_{4}\right)$ and concentrated onto silica gel. The residue was immediately purified by flash chromatography eluting with diethyl ether-hexanes mixtures. 
Palladacyclization of 2 with Stoichiometric $\operatorname{Pd}(0) \cdot(R)$-BINAP. Following GP1, a solution of aryl triflate 2 (50 mg, $85 \square \mathrm{mol}), \mathrm{Pd}(\mathrm{OAc})_{2}(19 \mathrm{mg}, 85 \square \mathrm{mol}),(R)$-BINAP (106 mg, 170 $\square \mathrm{mol})$, PMP (53 mg, $340 \square \mathrm{mol})$ and DMA $(0.9 \mathrm{~mL})$ was maintained at $80{ }^{\circ} \mathrm{C}$ for $14 \mathrm{~h}$. After the usual workup, the residue was purified by flash chromatography (50\% diethyl ether-hexanes)

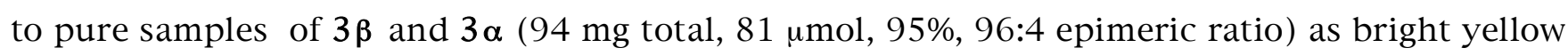
powders:

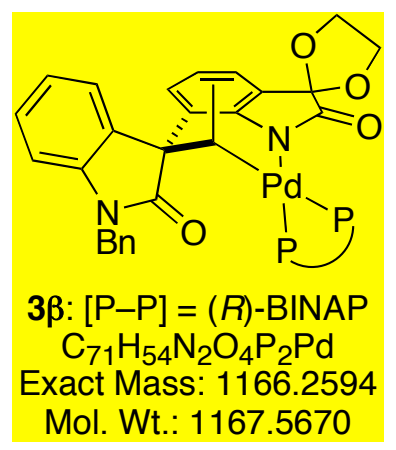

( $R$ )-BINAP Palladacycle (3马). $\mathrm{mp}>150{ }^{\circ} \mathrm{C}$, decomposition ( $\mathrm{Et}_{2} \mathrm{O}$-hexanes); ${ }^{1} \mathrm{H}$ NMR (500 MHz, THF-d8) $\square 9.15$ (t, $J=8.6 \mathrm{~Hz}, 1 \mathrm{H}, \operatorname{Ar} H$ ), 8.84 ( br s, 1H, ArH), 7.93-7.75 (m, 3H, ArH), 7.67-7.37 (m, 33H, ArH), 7.28-7.19 (m, 2H, ArH), 6.31 (d, J=7.6 Hz, 2H, ArH), 5.82 (br s, 1H, $\operatorname{Ar} H), 5.57-5.47\left(\mathrm{~m}, 2 \mathrm{H}, \mathrm{ArH}, \mathrm{CH}_{2}\right) 4.94\left(\mathrm{~d}, \mathrm{~J}=15.2 \mathrm{~Hz}, 1 \mathrm{H}, \mathrm{CH}_{2}\right), 4.40 \square 4.33\left(\mathrm{~m}, 1 \mathrm{H}, \mathrm{CH}_{2}\right), 4.02-$ $3.94\left(\mathrm{~m}, 1 \mathrm{H}, \mathrm{CH}_{2}\right), 3.85 \square 3.77\left(\mathrm{~m}, 1 \mathrm{H}, \mathrm{CH}_{2}\right), 3.68-3.60\left(\mathrm{~m}, 1 \mathrm{H}, \mathrm{CH}_{2}\right), 1.52\left(\mathrm{ddq},{ }^{3} J(\mathrm{H}, \mathrm{P})=20.2,6.7\right.$ $\left.\mathrm{Hz},{ }^{3} J(\mathrm{H}, \mathrm{H})=6.7 \mathrm{~Hz}, 1 \mathrm{H}, \mathrm{CH}\right), 0.88\left(\mathrm{ddd},{ }^{3} J(\mathrm{H}, \mathrm{H})=6.7 \mathrm{~Hz},{ }^{4} J(\mathrm{H}, \mathrm{P})=8.4,3.3 \mathrm{~Hz}, 3 \mathrm{H}, \mathrm{CH}_{3}\right) ;{ }^{13} \mathrm{C}$ NMR (125 MHz, THF-d8) $181.43\left(\mathrm{~d},{ }^{3} J(\mathrm{C}, \mathrm{P})=3.2 \mathrm{~Hz}\right), 181.08\left(\mathrm{~d},{ }^{4} J(\mathrm{C}, \mathrm{P})=1.9 \mathrm{~Hz}\right), 154.47$, $143.77,142.81(\mathrm{~d}, J(\mathrm{C}, \mathrm{P})=12.0 \mathrm{~Hz}), 138.60,138.32,138.19,137.32(\mathrm{dd}, J(\mathrm{C}, \mathrm{P})=7.4,3.7 \mathrm{~Hz})$, $136.83,136.73,135.92,135.66,135.99,135.39,135.21-134.60$ (m, 5C), 133.60, 133.55, 132.99, 132.76, 132.61, 132.51, 132.03, 131.81-131.49 (m, 3C), 130.73, 130.47, 130.09, $129.62,129.42,129.33,129.15,129.07,128.94,128.86,128.80,128.73,128.67,128.57$, $128.48,128.43,128.38,128.30,128.17,127.89,127.83,127.69,127.44,127.22,126.86$, $126.04,125.95,125.40,124.33,123.32,122.39,118.90,109.44,105.15,79.64,65.67,64.36$, $60.97(\mathrm{br}), 44.60,44.28\left(\mathrm{dd},{ }^{2} J(\mathrm{C}, \mathrm{P})=99.4,6.5 \mathrm{~Hz}\right), 19.83\left(\mathrm{dd},{ }^{3} J(\mathrm{C}, \mathrm{P})=4.2,1.9 \mathrm{~Hz}\right) ;{ }^{31} \mathrm{P}$ NMR

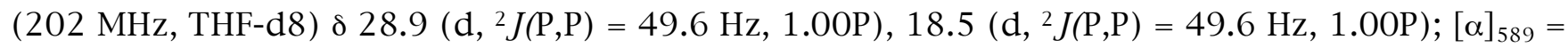


+191, $[\square]_{577}=+208,[\square]_{546}=+282,[\square]_{435}$ and $[\square]_{405}$ not possible due to sample absorbance $(c=$ 0.41 in $\mathrm{CHCl}_{3}$ ); IR (thin film) 3053, 2957, 2926, 2853, 1695, 1668, 1594, 1482, 1432, 1339, 1293, 1177, 1123, 1096, 1000, 942, 911, 815, 741, 695 cm $\square 1$; LRMS (ESI+) Calcd for $\mathrm{C}_{71} \mathrm{H}_{55} \mathrm{~N}_{2} \mathrm{O}_{4} \mathrm{P}_{2} \mathrm{Pd}(\mathrm{M}+\mathrm{H})^{+}:$1167.3. Found: 1167.1 .
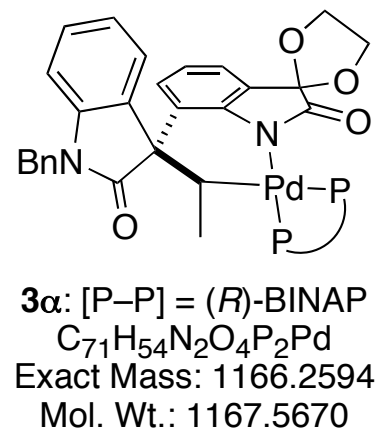

(R)-BINAP palladacycle (3q). $\mathrm{mp}>160{ }^{\circ} \mathrm{C}$, decomposition ( $\mathrm{Et}_{2} \mathrm{O}$-hexanes); ${ }^{1} \mathrm{H}$ NMR $\left(500 \mathrm{MHz}, \mathrm{CDCl}_{3}\right) \square 8.36(\mathrm{~d}, J=7.0 \mathrm{~Hz} 1 \mathrm{H}, \mathrm{ArH}), 7.85 \square 7.37(\mathrm{~m}, 22 \mathrm{H}, \mathrm{ArH}), 7.12 \square 7.03(\mathrm{~m}, 7 \mathrm{H}$, $\operatorname{Ar} H), 6.90 \square 6.82(\mathrm{~m}, 5 \mathrm{H}, \operatorname{Ar} H), 6.71 \square 6.67(\mathrm{~m}, 2 \mathrm{H}, \operatorname{Ar} H), 6.57 \square 6.53(\mathrm{dd}, J=12.7,7.6 \mathrm{~Hz}, 2 \mathrm{H}, \operatorname{Ar} H)$, $6.42(\mathrm{t}, J=7.4 \mathrm{~Hz}, 1 \mathrm{H}, \operatorname{Ar} H), 6.39(\mathrm{t}, J=7.4 \mathrm{~Hz}, 1 \mathrm{H}, \operatorname{Ar} H), 6.16$ (br s, $1 \mathrm{H}, \operatorname{Ar} H), 5.97$ (br s, $1 \mathrm{H}$, $\operatorname{Ar} H), 5.58(\mathrm{~d}, J=8.6 \mathrm{~Hz}, 1 \mathrm{H}, \operatorname{Ar} H), 5.16\left(\mathrm{~d}, J=16.0 \mathrm{~Hz}, 1 \mathrm{H}, \mathrm{CH}_{2}\right), 4.45\left(\mathrm{~d}, J=16.0 \mathrm{~Hz}, 1 \mathrm{H}, \mathrm{CH}_{2}\right)$, $4.26 \square 4.20\left(\mathrm{~m}, 1 \mathrm{H}, \mathrm{CH}_{2}\right), 4.01\left(\mathrm{q}, J=6.9 \mathrm{~Hz}, 1 \mathrm{H}, \mathrm{CH} \mathrm{H}_{2}\right), 3.84 \square 3.81\left(\mathrm{~m}, 1 \mathrm{H}, \mathrm{CH}_{2}\right), 3.57(\mathrm{q}, J=7.2$, $\left.1 \mathrm{H}, \mathrm{C} H_{2}\right), 2.02\left(\mathrm{qdd},{ }^{1} J(\mathrm{H}, \mathrm{H})=6.6 \mathrm{~Hz},{ }^{3} J(\mathrm{H}, \mathrm{P})=5.9 \mathrm{~Hz},{ }^{3} J(\mathrm{H}, \mathrm{P})=1.4 \mathrm{~Hz}, 1 \mathrm{H}, \mathrm{CH}\right), 0.00(\mathrm{ddd}$, $\left.{ }^{3} J(\mathrm{H}, \mathrm{P})=12.4 \mathrm{~Hz},{ }^{1} J(\mathrm{H}, \mathrm{H})=6.6 \mathrm{~Hz},{ }^{3} J(\mathrm{H}, \mathrm{P})=2.8 \mathrm{~Hz}, 3 \mathrm{H}, \mathrm{CH}_{3}\right) ;{ }^{13} \mathrm{C} \mathrm{NMR}\left(125 \mathrm{MHz}, \mathrm{CDCl}_{3}\right) \square$ $180.76\left(\mathrm{~d},{ }^{4} J(\mathrm{C}, \mathrm{P})=3.8 \mathrm{~Hz}\right), 178.30\left(\mathrm{dd},{ }^{3} J(\mathrm{C}, \mathrm{P})=13.8,1.3 \mathrm{~Hz}\right), 149.74,142.83,142.72,141.82$, $137.40,136.37,135.81,135.60,135.28,135.18,134.44,134.37,134.03,133.99,133.94$, 133.90, 133.54, 133.46, 133.07, 131.58, 131.33, $130.91(\mathrm{~d}, J(\mathrm{C}, \mathrm{P})=3.8 \mathrm{~Hz}), 130.85,130.54(\mathrm{~d}$, $J(\mathrm{C}, \mathrm{P})=3.2 \mathrm{~Hz}), 130.28(\mathrm{~d}, J(\mathrm{C}, \mathrm{P})=1.8 \mathrm{~Hz}), 130.14(\mathrm{~d}, J(\mathrm{C}, \mathrm{P})=2.3 \mathrm{~Hz}), 129.42,129.00(2 \mathrm{C})$, 128.90, $128.86(\mathrm{~d}, J(\mathrm{C}, \mathrm{P})=1.6 \mathrm{~Hz}), 128.83(2 \mathrm{C}), 128.43(2 \mathrm{C}), 128.35(\mathrm{~d}, J(\mathrm{C}, \mathrm{P})=1.9 \mathrm{~Hz})$, $128.14,128.07,127.99,127.92,127.87,127.81,127.79,127.61,127.46,127.32,127.21$, $127.15,127.10,127.05,127.02,126.81$ (2C), 126.60, 126.49, 126.25, 126.16 (2C), 125.98, $125.42,123.30,122.43,120.24,65.60,64.31,59.89\left(\mathrm{dd},{ }^{3} J(\mathrm{C}, \mathrm{P})=4.2,1.4 \mathrm{~Hz}\right), 43.12,40.99$ $\left(\mathrm{dd},{ }^{2} J(\mathrm{C}, \mathrm{P})=103.2,4.3 \mathrm{~Hz}\right), 14.90\left(\mathrm{dd},{ }^{3} J(\mathrm{C}, \mathrm{P})=4.6,2.3 \mathrm{~Hz}\right) ;{ }^{31} \mathrm{P} \mathrm{NMR}\left(202 \mathrm{MHz}, \mathrm{CDCl}_{3}\right) \square$ 
$22.20\left(\mathrm{~d},{ }^{2} J(\mathrm{P}, \mathrm{P})=44.1 \mathrm{~Hz}, 1.00 \mathrm{P}\right), 19.40\left(\mathrm{~d},{ }^{2} J(\mathrm{P}, \mathrm{P})=44.1 \mathrm{~Hz}, 0.96 \mathrm{P}\right) ;[\square]_{589}=+203,[\square]_{577}=$ +199, $[\square]_{546},[\square]_{435}$ and $[\square]_{405}$ not possible due to sample absorbance $\left(c=0.46\right.$ in $\mathrm{CHCl}_{3}$ ); IR (thin film) 3057, 2957, 2926, 2856, 1706, 1671, 1610, 1482, 1436, 1343, 1312, 1292, 1181, 1127, 1096, 1077, 1004, 946, 745, $695 \mathrm{~cm}^{\square 1}$; LRMS (ESI+) Calcd for $\mathrm{C}_{71} \mathrm{H}_{55} \mathrm{~N}_{2} \mathrm{O}_{4} \mathrm{P}_{2} \mathrm{Pd}(\mathrm{M}+\mathrm{H})^{+}:$1167.3. Found: 1167.5. X-ray quality crystals were obtained from a solution of $3 \square$ in $\mathrm{MeOH}$ (See Supporting Information, Part II).

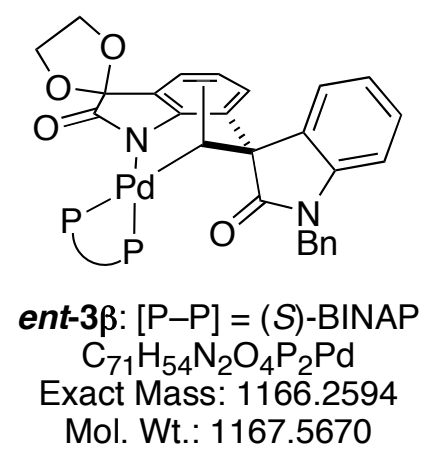

(S)-BINAP Palladacycle (ent-30). Following GP1, a solution of aryl triflate 2 (50 mg, $85 \square \mathrm{mol}), \operatorname{Pd}(\mathrm{OAc})_{2}(19 \mathrm{mg}, 85 \square \mathrm{mol}),(S)$-BINAP (106 mg, $\left.170 \square \mathrm{mol}\right)$, PMP (53 mg, $\left.334 \square \mathrm{mol}\right)$ and DMA $(0.9 \mathrm{~mL})$ was maintained at $80{ }^{\circ} \mathrm{C}$ for $16 \mathrm{~h}$. After the usual workup, the residue was purified by silica gel flash chromatography (50\% diethyl ether-hexanes) to afford ent-30 (83 mg, $71 \square \mathrm{mol}, 84 \%)$ as a bright yellow solid: $\mathrm{mp}>150{ }^{\circ} \mathrm{C}$, decomposition $\left(\right.$ Et $_{2} \mathrm{O}$-hexanes); ${ }^{1} \mathrm{H}$ NMR $\left(500 \mathrm{MHz}, \mathrm{CDCl}_{3}\right) \square 9.07(\mathrm{t}, J=9.1 \mathrm{~Hz}, 1 \mathrm{H}, \mathrm{Ar} H), 8.72(\mathrm{br} \mathrm{s}, 1 \mathrm{H}, \mathrm{Ar} H), 7.87-7.77(\mathrm{~m}, 3 \mathrm{H}, \mathrm{ArH})$, 7.60-7.26 (m, 15H, ArH), 7.24-6.93 (m, 11H, ArH), 6.89 (d, J=7.0 Hz, 1H, ArH), 6.83-6.74 (m, 3H, ArH), 6.65-6.47 (m, 6H, ArH), 6.31-6.24 (m, 2H, ArH), $5.78(\mathrm{t}, J=7.0 \mathrm{~Hz}, 1 \mathrm{H}, \mathrm{ArH}), 5.39(\mathrm{~d}$, $\left.J=15.2 \mathrm{~Hz}, 1 \mathrm{H}, \mathrm{CH}_{2}\right) 4.90\left(\mathrm{~d}, J=15.2 \mathrm{~Hz}, 1 \mathrm{H}, \mathrm{CH}_{2}\right), 4.44(\mathrm{td}, J=7.0,3.8 \mathrm{~Hz}, 1 \mathrm{H}, \mathrm{CH}), 4.12(\mathrm{q}, J$ $\left.=7.1 \mathrm{~Hz}, 1 \mathrm{H}, \mathrm{C} H_{2}\right), 3.96\left(\mathrm{td}, J=7.1,3.8 \mathrm{~Hz}, 1 \mathrm{H}, \mathrm{C} H_{2}\right), 3.72\left(\mathrm{q}, J=7.3,1 \mathrm{H}, \mathrm{CH}_{2}\right), 1.45(\mathrm{ddq}$, $\left.{ }^{3} J(\mathrm{H}, \mathrm{P})=20.2,6.7 \mathrm{~Hz},{ }^{3} J(\mathrm{H}, \mathrm{H})=6.7 \mathrm{~Hz}, 1 \mathrm{H}, \mathrm{CH}\right), 0.89\left(\mathrm{ddd},{ }^{3} J(\mathrm{H}, \mathrm{H})=6.7 \mathrm{~Hz},{ }^{4} J(\mathrm{H}, \mathrm{P})=8.4,3.3\right.$ $\left.\mathrm{Hz}, 3 \mathrm{H}, \mathrm{CH}_{3}\right) ;{ }^{13} \mathrm{C} \mathrm{NMR}\left(125 \mathrm{MHz}, \mathrm{CDCl}_{3}\right) \square 181.07\left(\mathrm{~d},{ }^{4} \mathrm{~J}(\mathrm{C}, \mathrm{P})=2.8 \mathrm{~Hz}\right), 180.57\left(\mathrm{dd},{ }^{3} J(\mathrm{C}, \mathrm{P})=2.8\right.$, $0.5 \mathrm{~Hz}), 152.95,142.58,141.88(\mathrm{dd}, J(\mathrm{C}, \mathrm{P})=9.9,2.1 \mathrm{~Hz}), 137.05,136.78,136.56,136.25$, $135.78,135.68,134.54,134.49,134.21,134.15,133.95,133.82,133.53,133.45(\mathrm{~d}, J(\mathrm{C}, \mathrm{P})=$ $1.4 \mathrm{~Hz}), 132.76,132.57,132.52,131.98,131.75,131.39,131.29,130.91,130.84,130.60$, 
$130.44,129.53,129.18,129.04$ (2C), 128.85, 128.77, $128.50,128.44,128.28,128.25$ (2C), $128.15,128.03,127.97,127.85,127.76,127.73,127.60,127.55,127.25,127.13,127.00$, $126.52,126.44,126.12,126.08,125.45,125.20,124.85,123.85,122.27,121.92,119.16$, 108.37, $104.55\left(\mathrm{dd},{ }^{4} J(\mathrm{C}, \mathrm{P})=4.9,1.2 \mathrm{~Hz}\right), 65.78,64.10,60.21,44.34,43.78\left(\mathrm{dd},{ }^{2} J(\mathrm{C}, \mathrm{P})=98.8\right.$, $6.7 \mathrm{~Hz}), 19.46\left(\mathrm{dd},{ }^{3} J(\mathrm{C}, \mathrm{P})=2.8,0.9 \mathrm{~Hz}\right) ;{ }^{31} \mathrm{P} \mathrm{NMR}\left(162 \mathrm{MHz}, \mathrm{CDCl}_{3}\right) \square 29.5\left(\mathrm{~d},{ }^{2} J(\mathrm{P}, \mathrm{P})=51.7 \mathrm{~Hz}\right.$,

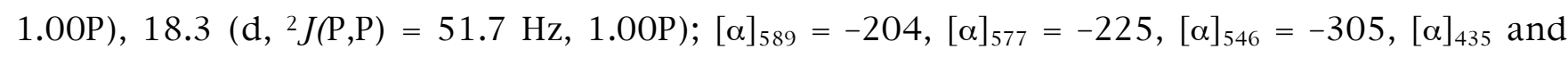
$[\square]_{405}$ not possible due to sample absorbance $\left(c=2.0\right.$ in $\mathrm{CHCl}_{3}$ ); IR (thin film) 3053, 2958, 2925 , 2853, 1695, 1667, 1594, 1482, 1432, 1339, 1292, 1177, 1123, 1096, 1000, 942, 911, 815, 741, $695 \mathrm{~cm}^{\square 1}$; LRMS (ESI) Calcd for $\mathrm{C}_{71} \mathrm{H}_{55} \mathrm{~N}_{2} \mathrm{O}_{4} \mathrm{P}_{2} \mathrm{Pd}(\mathrm{M}+\mathrm{H})^{+}:$1167.3. Found: 1167.6.

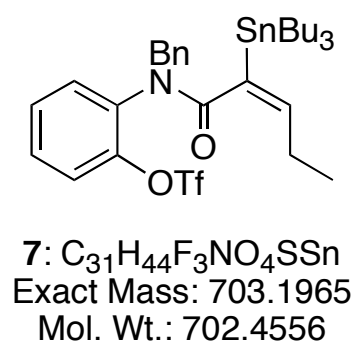

Trifluoromethanesulfonic acid (E)-2-\{benzyl-[2-(tributylstannanyl)-pent-2enoyl]-amino\} phenyl ester (7). n-BuLi (0.52 mL, $1.33 \mathrm{mmol}, 2.57 \mathrm{M}$ in hexanes) was added drop wise to a solution of 1-butyne $(719 \mathrm{mg}, 13.3 \mathrm{mmol})$ and THF $(7 \mathrm{~mL})$ at $-78^{\circ} \mathrm{C}$. After $30 \mathrm{~min}$, a solution of 3-benzyl-3H-benzooxazolan-2-one 5 (300 mg, $1.33 \mathrm{mmol}$ ) and THF (2 mL) was added in one portion at $-78{ }^{\circ} \mathrm{C}$. The reaction mixture was warmed to $-10{ }^{\circ} \mathrm{C}$ and maintained for $14 \mathrm{~h}$. A solution of $\operatorname{PhNTf}_{2}(713 \mathrm{mg}, 2.00 \mathrm{mmol})$ and THF $(3 \mathrm{~mL})$ was added, and the reaction mixture was stirred at ambient temperature for $6 \mathrm{~h}$ after which time it was poured into $\mathrm{H}_{2} \mathrm{O}(20 \mathrm{~mL})$. The organic layer was separated, and the aqueous layer was extracted with EtOAc $(60 \mathrm{~mL})$. The combined organic extracts were dried $\left(\mathrm{Na}_{2} \mathrm{SO}_{4}\right)$, filtered, and concentrated. The residue was purified by silica gel chromatography (20\% ethyl acetate-hexanes) to afford 6 (306 $\mathrm{mg}, 0.75 \mathrm{mmol}, 56 \%)$ as an off-white solid. Alkyne 6 was immediately allowed to react with tributyltinhydride to afford 7. Diagnostic data for intermediate alkyne 6: ${ }^{1} \mathrm{H} \mathrm{NMR}(500 \mathrm{MHz}$, $\left.\mathrm{CDCl}_{3}\right) \mathrm{7} .50-7.15(\mathrm{~m}, 8 \mathrm{H}, \mathrm{ArH}), 7.05-6.96(\mathrm{~m}, 1 \mathrm{H}, \mathrm{ArH}), 5.66(\mathrm{~d}, J=14.6 \mathrm{~Hz}, 1 \mathrm{H}, \mathrm{CH}), 4.20(\mathrm{~d}$, 
$\left.J=14.7 \mathrm{~Hz}, 1 \mathrm{H}, \mathrm{CH}_{2}\right), 2.30-2.22\left(\mathrm{~m}, 2 \mathrm{H}, \mathrm{CH}_{2}\right), 1.02\left(\mathrm{t}, J=7.4 \mathrm{~Hz}, 3 \mathrm{H}, \mathrm{CH}_{3}\right)$; LRMS (ESI+): 412.2 $(\mathrm{M}+\mathrm{H})^{+}, 434.1(\mathrm{M}+\mathrm{Na})^{+}, 450.2(\mathrm{M}+\mathrm{K})^{+}$.

A solution of tributyltinhydride $(212 \mathrm{mg}, 729$ 口mol) and THF (1.5 mL, BHT-inhibited, sparged with Ar for $30 \mathrm{~min}$ ) was slowly added drop wise to a solution of 6 (250 mg, $608 \square \mathrm{mol})$, $\mathrm{Pd}\left(\mathrm{PPh}_{3}\right)_{4}(42 \mathrm{mg}, 36 \square \mathrm{mol}, 6 \mathrm{~mol} \%)$ and THF (1.2 mL, BHT-inhibited, sparged with Ar for 30 min) at $0{ }^{\circ} \mathrm{C}$. The solution was maintained at $0{ }^{\circ} \mathrm{C}$ for $1 \mathrm{~h}$, concentrated onto silica gel, and purified by silica gel chromatography (10\% ethyl acetate-hexanes) to afford 7 (390 mg, 553 $\square \mathrm{mol}, 91 \%)$ as a colorless oil. Note: Because of amide rotational isomers, NMR spectra are complex, therefore integrations are omitted and only peaks listed. ${ }^{1} \mathrm{H}$ NMR $\left(500 \mathrm{MHz}, \mathrm{CDCl}_{3}\right) \square$ 7.37-7.06 (m), 6.80-6.63 (m), $6.14(\mathrm{br} \mathrm{s}), 5.97-5.66(\mathrm{~m}), 5.32(\mathrm{t}, J=6.2 \mathrm{~Hz}), 4.90$ (br s), 4.26$3.85(\mathrm{~m}), 3.74(\mathrm{t}, J=5.4 \mathrm{~Hz}), 2.36(\mathrm{br} \mathrm{s}), 2.04(\mathrm{br} \mathrm{s}), 1.95-1.67(\mathrm{~m}), 1.59-1.20(\mathrm{~m}), 1.12-0.60$ (m); ${ }^{13} \mathrm{C}$ NMR (125 MHz, $\left.\mathrm{CDCl}_{3}\right) \square 174.16,148.50,145.62,139.64,137.68,136.44,132.64$, 129.93-127.83 (7 C), 122.01, 52.68-51.70 (m, 1C), 29.21 (m, 3C), 28.92, 28.54, $27.52(\mathrm{~m}, 3 \mathrm{C})$, 13.77 (m, 3C), 11.37 (m, 3C); IR (thin film) 2961, 2930, 2872, 1640, 1602, 1494, 1455, 1424, $1374,1285,1247,1216,1143,1100,1081,884,768,737,699 \mathrm{~cm}^{\square 1}$; HRMS (ESI+) Calcd for $\mathrm{C}_{31} \mathrm{H}_{44} \mathrm{~F}_{3} \mathrm{NO}_{4}{ }^{116} \mathrm{SnSNa}(\mathrm{M}+\mathrm{Na})^{+}$: 722.1859. Found: 722.1871; Calcd for $\mathrm{C}_{31} \mathrm{H}_{45} \mathrm{~F}_{3} \mathrm{NO}_{4}{ }^{116} \mathrm{SnS}(\mathrm{M}+\mathrm{H})^{+}$: 700.2039. Found: 700.2036.

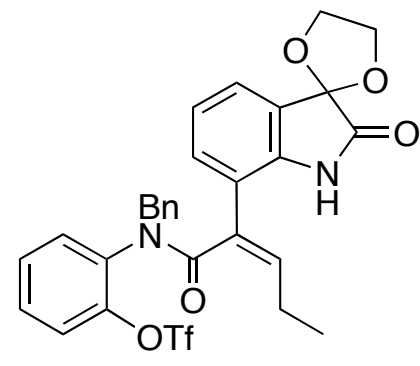

9: $\mathrm{C}_{29} \mathrm{H}_{25} \mathrm{~F}_{3} \mathrm{~N}_{2} \mathrm{O}_{7} \mathrm{~S}$

Exact Mass: 602.1335

Mol. Wt.: 602.5782 
Trifluoromethanesulfonic acid (Z)-2-\{benzyl-[2-(2-oxo-3,3-spiro[1,3dioxolane]-2,3-dihydro- $1 H$-indol-7-yl)-pent-2-enoyl]-amino\}-phenyl ester (9). ${ }^{3}$ An Air-free ${ }^{\circledR}$ flask was charged with $\mathrm{Pd}_{2} \mathrm{dba}_{3} \cdot \mathrm{CHCl}_{3}(115 \mathrm{mg}, 111 \square \mathrm{mol}), \mathrm{P}(2$-furyl) 3 (158 mg, 666 $\square \mathrm{mol}$ ) and freshly distilled $\mathrm{N}$-methylpyrolidinone (NMP, $8 \mathrm{~mL}$ ). The mixture was deoxygenated by bubbling Ar through a submerged needle for $2 \mathrm{hr}$, during which time a bright yellow solution resulted. A deoxygenated solution of stannane 7 (390 mg, 555 [mol), iodide 8 (194 mg, $611 \square \mathrm{mol}$ ) and NMP (3 mL) was then added via syringe. Copper(I) iodide (211 mg, 1.11 mmol) was added and the resulting reaction mixture was flushed with a stream of Ar for 15 min, sealed and stirred at $\mathrm{rt}$ for $24 \mathrm{~h}$. After diluting the reaction with EtOAc $(100 \mathrm{~mL}), \mathrm{KF}$ was added until the mixture became transparent yellow. The mixture was filtered and the filtrate was washed with $\mathrm{H}_{2} \mathrm{O}(50 \mathrm{~mL})$ and brine $(50 \mathrm{~mL})$. The organic layer was dried $\left(\mathrm{Na}_{2} \mathrm{SO}_{4}\right)$, filtered and concentrated. The crude residue was purified by flash chromatography (10\% ethyl acetatehexanes) to give $14(211 \mathrm{mg}, 350 \square \mathrm{mol}, 63 \%)$ as an off-white solid: $\mathrm{mp} 150-152{ }^{\circ} \mathrm{C}(\mathrm{MeOH}) ;{ }^{1} \mathrm{H}$ NMR (500 MHz, $\left.\mathrm{CDCl}_{3}\right) \square 7.83($ br s, $1 \mathrm{H}, \mathrm{NH}), 7.31-7.20(\mathrm{~m}, 7 \mathrm{H}, \mathrm{ArH}), 7.14(\mathrm{~d}, J=7.2 \mathrm{~Hz}, 1 \mathrm{H}$, $\operatorname{Ar} H), 6.91(\mathrm{td}, J=7.7,1.3 \mathrm{~Hz}, 1 \mathrm{H}, \operatorname{Ar} H), 6.79(\mathrm{t}, J=7.6 \mathrm{~Hz}, 1 \mathrm{H}, \operatorname{Ar} H), 6.56(\mathrm{dd}, J=8.0,0.8 \mathrm{~Hz}$, 1H, $\operatorname{Ar} H), 6.21(\mathrm{~d}, J=7.7 \mathrm{~Hz}, 1 \mathrm{H}, \operatorname{Ar} H), 5.90\left(\mathrm{~d}, J=14.3 \mathrm{~Hz}, 1 \mathrm{H}, \mathrm{C} H_{2}\right), 5.49(\mathrm{t}, J=7.6 \mathrm{~Hz}, 1 \mathrm{H}$, $\mathrm{CH}), 4.52-4.44\left(\mathrm{~m}, 2 \mathrm{H}, \mathrm{CH}_{2}\right), 4.27 \square 4.22\left(\mathrm{~m}, 2 \mathrm{H}, \mathrm{CH}_{2}\right), 3.90\left(\mathrm{~d}, \mathrm{~J}=14.3 \mathrm{~Hz}, 1 \mathrm{H}, \mathrm{CH}_{2}\right), 2.32-2.24$ (m, 2H, $\left.\mathrm{CH}_{2}\right), 1.04\left(\mathrm{t}, J=7.5 \mathrm{~Hz}, 3 \mathrm{H}, \mathrm{CH}_{3}\right) ;{ }^{13} \mathrm{C} \mathrm{NMR}\left(125 \mathrm{MHz}, \mathrm{CDCl}_{3}\right) \square$ 174.34, 169.28, 145.43, 140.03 (br), 139.69, 136.63, 133.32, 132.83, 132.00, 130.59, 130.38, 129.46 (2C), 128.79 (2C), $128.32,128.18,124.89,124.40,123.03,121.99,120.99,118.48\left(\mathrm{q}, J_{\mathrm{C}, \mathrm{F}}=320.0 \mathrm{~Hz}\right), 101.93$, 65.99, 65.76, 52.18, 23.62, 13.62; IR (thin film) 3223, 2976, 2937, 2903, 1733, 1656, 1613, $1494,1447,1420,1324,1278,1216,1185,1139,1104,1031,1007,946,892,853,749,703$ $\mathrm{cm}^{\square 1}$; HRMS (ESI+) Calcd for $\mathrm{C}_{29} \mathrm{H}_{25} \mathrm{~F}_{3} \mathrm{~N}_{2} \mathrm{O}_{7} \mathrm{SNa}(\mathrm{M}+\mathrm{Na})^{+}:$602.1232. Found: 625.1243. Anal. Calcd for $\mathrm{C}_{29} \mathrm{H}_{25} \mathrm{~F}_{3} \mathrm{~N}_{2} \mathrm{O}_{7} \mathrm{~S}: \mathrm{C}, 57.80 ; \mathrm{H}, 4.18 ; \mathrm{N}, 4.65$. Found: C, 57.63; H, 4.04; N, 4.64.

\footnotetext{
3 The conditions used for Stille coupling were described: Garden, S. J.; Torres, J. C.; da Silva, L. E.; Pinto, A. C. Syn. Commun. 1998, 28, 1679-1689.
} 


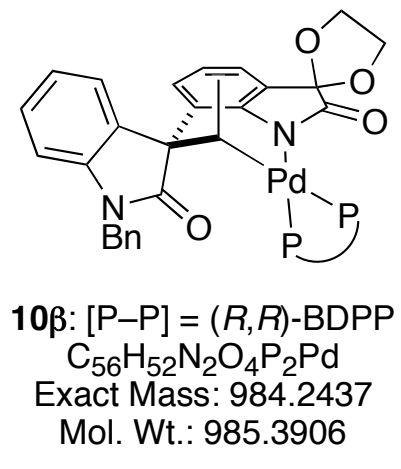

$(R, R)$-BDPP Palladacycle (10马). Following GP1, a solution of aryl triflate 2 (75 mg, $127 \square \mathrm{mol}), \mathrm{Pd}(\mathrm{OAc})_{2}(29 \mathrm{mg}, 127 \square \mathrm{mol}),(2 R, 4 R)-(+)-2,4-\mathrm{bis}($ diphenylphosphino)pentane $[(R, R)-$

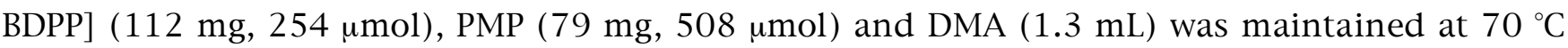
for $16 \mathrm{~h}$. After the usual workup, the residue was purified by flash chromatography (50\% diethyl ether-hexanes) to afford $10 \square(118 \mathrm{mg}, 119 \square \mathrm{mol}, 94 \%)$ as a colorless solid: $\mathrm{mp}>170{ }^{\circ} \mathrm{C}$, decomposition (Et ${ }_{2} \mathrm{O}$-hexanes); ${ }^{1} \mathrm{H}$ NMR (500 MHz, $\left.\mathrm{CDCl}_{3}\right) \square 8.18$ (br m, 2H, ArH), 7.77 (t, $J=8.8$ $\mathrm{Hz}, 2 \mathrm{H}, \operatorname{Ar} H), 7.59$ (t, $J=8.9 \mathrm{~Hz}, 2 \mathrm{H}, \operatorname{Ar} H), 7.39$ (d, J=7.4 Hz, 2H, ArH), $7.31 \square 7.12(\mathrm{~m}, 12 \mathrm{H}$, $\operatorname{Ar} H), 7.02 \square 6.89(\mathrm{~m}, 6 \mathrm{H}, \operatorname{Ar} H), 6.77 \square 6.74(\mathrm{~m}, 3 \mathrm{H}, \operatorname{Ar} H), 6.67(\mathrm{~d}, J=7.8 \mathrm{~Hz}, 1 \mathrm{H}, \operatorname{Ar} H), 6.49(\mathrm{t}, J=$ $7.6 \mathrm{~Hz}, 1 \mathrm{H}, \operatorname{Ar} H), 6.27$ (d, $J=7.9 \mathrm{~Hz}, 1 \mathrm{H}, \operatorname{Ar} H), 5.27\left(\mathrm{~d}, J=15.3 \mathrm{~Hz}, 1 \mathrm{H}, \mathrm{CH}_{2}\right), 4.62(\mathrm{~d}, J=15.3$ $\left.\mathrm{Hz}, 1 \mathrm{H}, \mathrm{CH}_{2}\right), 4.20 \square 4.16\left(\mathrm{~m}, 1 \mathrm{H}, \mathrm{CH}_{2}\right), 3.90\left(\mathrm{dd}, J=13.8,6.9 \mathrm{~Hz}, 1 \mathrm{H}, \mathrm{CH}_{2}\right), 3.71 \square 3.67(\mathrm{~m}, 1 \mathrm{H}$, $\left.\mathrm{CH}_{2}\right), 3.51\left(\mathrm{dd}, J=13.8,7.3,1 \mathrm{H}, \mathrm{CH}_{2}\right), 2.82 \square 2.72(\mathrm{~m}, 1 \mathrm{H}, \mathrm{CH}), 2.71 \square 2.61(\mathrm{~m}, 1 \mathrm{H}, \mathrm{CH}), 2.00 \square 1.81$ (m, 2H, $\left.\mathrm{CH}_{2}\right), 1.59 \square 1.51\left(\mathrm{~m}, 4 \mathrm{H}, \mathrm{CH}_{3}, \mathrm{CH}\right), 1.07$ (ddd, $\left.J=10.5,6.9,3.8 \mathrm{~Hz}, 3 \mathrm{H}, \mathrm{CH}_{3}\right), 0.94(\mathrm{dd}, J$ $\left.=10.7,7.2 \mathrm{~Hz}, 3 \mathrm{H}, \mathrm{CH}_{3}\right) ;{ }^{13} \mathrm{C} \mathrm{NMR}\left(125 \mathrm{MHz}, \mathrm{CDCl}_{3}\right) \square 180.54\left(\mathrm{dd},{ }^{3} J(\mathrm{C}, \mathrm{P})=2.4,0.5 \mathrm{~Hz}\right), 180.32$ $\left(\mathrm{d},{ }^{4} J(\mathrm{C}, \mathrm{P})=1.8 \mathrm{~Hz}\right), 152.84,142.36,136.69,136.62(2 \mathrm{C}), 136.52,135.05,134.94,134.30$, 134.05, 132.08, 131.99, 131.70, 131.64, 131.23, 130.87, 130.45, 130.22 (m, 3C), $128.89 \square 127.76(\mathrm{~m}, 14 \mathrm{C}), 126.72,124.82,124.03,122.24,121.66,119.15,108.26,104.25$, 104.21, 65.19, 64.38, 59.73, 44.23, $42.30\left(\mathrm{dd},{ }^{2} J(\mathrm{C}, \mathrm{P})=95.3,6.3 \mathrm{~Hz}\right), 35.97$ (br), 29.29 (dd, $J(\mathrm{C}, \mathrm{P})=19.9,1.4 \mathrm{~Hz}), 25.84(\mathrm{dd}, J(\mathrm{C}, \mathrm{P})=23.1,3.2 \mathrm{~Hz}), 20.15\left(\mathrm{dd},{ }^{3} J(\mathrm{C}, \mathrm{P})=7.9,1.0 \mathrm{~Hz}\right), 18.62$ $(\mathrm{dd}, J(\mathrm{C}, \mathrm{P})=4.2,2.3 \mathrm{~Hz}), 15.66(\mathrm{~d}, J(\mathrm{C}, \mathrm{P})=8.8 \mathrm{~Hz}) ;{ }^{11} \mathrm{P}$ NMR $\left(162 \mathrm{MHz}, \mathrm{CDCl}_{3}\right) \square 28.50\left(\mathrm{~d}, J_{\mathrm{P}, \mathrm{P}}=\right.$ 


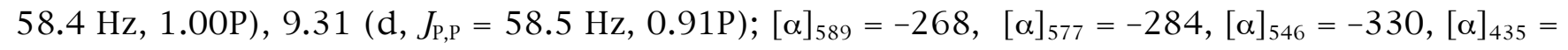
-684, [u] $]_{405}=-918\left(c=1.0\right.$ in $\left.\mathrm{CHCl}_{3}\right)$; IR (thin film) 3057, 2964, 2930, 2899, 2853, 1698, 1671, 1598, 1486, 1467, 1432, 1343, 1297, 1235, 1181, 1127, 1100, 1000, 942, 745, 699 cm ${ }^{\square 1}$; LRMS (ESI+) Calcd for $\mathrm{C}_{56} \mathrm{H}_{53} \mathrm{~N}_{2} \mathrm{O}_{4} \mathrm{P}_{2} \mathrm{Pd}(\mathrm{M}+\mathrm{H})^{+}$: 985.25. Found: 985.28. X-ray quality crystals were obtained from a solution of $10 \mathrm{C}$ in $\mathrm{Et}_{2} \mathrm{O}$ (See Supporting Information II).

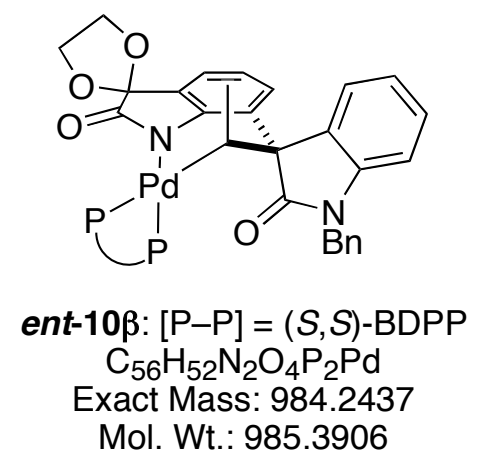

$(S, S)$-BDPP Palladacycle (ent-10 $)$. Following GP1, a solution of aryl triflate 2 (25 mg, $42 \square \mathrm{mol}), \mathrm{Pd}(\mathrm{OAc})_{2}(9.4 \mathrm{mg}, 42 \square \mathrm{mol}), \mathrm{PMP}(26 \mathrm{mg}, 170 \square \mathrm{mol})$ and DMA (0.5 mL) were maintained at $70{ }^{\circ} \mathrm{C}$ for $16 \mathrm{~h}$. After the usual workup, the residue was purified by flash chromatography (50\% diethyl ether-hexanes) to afford ent-10 (36 mg, $37 \square \mathrm{mol}, 89 \%)$ of as a colorless solid: $\mathrm{mp}>170{ }^{\circ} \mathrm{C}$, decomposition ( $\mathrm{Et}_{2} \mathrm{O}$-hexanes); ${ }^{1} \mathrm{H}$ NMR $\left(500 \mathrm{MHz}, \mathrm{CDCl}_{3}\right) \square 8.18$ (br m, 2H, ArH), 7.77 (t, $J=8.8 \mathrm{~Hz}, 2 \mathrm{H}, \operatorname{Ar} H), 7.59(\mathrm{t}, J=8.9 \mathrm{~Hz}, 2 \mathrm{H}, \operatorname{Ar} H), 7.39(\mathrm{~d}, J=7.4 \mathrm{~Hz}, 2 \mathrm{H}$, $\operatorname{Ar} H), 7.31 \square 7.12(\mathrm{~m}, 12 \mathrm{H}, \operatorname{Ar} H), 7.02 \square 6.89(\mathrm{~m}, 6 \mathrm{H}, \operatorname{Ar} H), 6.77 \square 6.74(\mathrm{~m}, 3 \mathrm{H}, \operatorname{Ar} H), 6.67(\mathrm{~d}, J=7.8$ $\mathrm{Hz}, 1 \mathrm{H}, \operatorname{Ar} H), 6.49$ (t, $J=7.6 \mathrm{~Hz}, 1 \mathrm{H}, \operatorname{Ar} H), 6.27(\mathrm{~d}, J=7.9 \mathrm{~Hz}, 1 \mathrm{H}, \operatorname{Ar} H), 5.27(\mathrm{~d}, J=15.3 \mathrm{~Hz}$, $\left.1 \mathrm{H}, \mathrm{CH}_{2}\right), 4.62\left(\mathrm{~d}, J=15.3 \mathrm{~Hz}, 1 \mathrm{H}, \mathrm{CH}_{2}\right), 4.20 \square 4.16\left(\mathrm{~m}, 1 \mathrm{H}, \mathrm{CH}_{2}\right), 3.90(\mathrm{dd}, J=13.8,6.9 \mathrm{~Hz}, 1 \mathrm{H}$, $\left.\mathrm{CH}_{2}\right), 3.71 \square 3.67\left(\mathrm{M}, 1 \mathrm{H}, \mathrm{CH}_{2}\right), 3.51\left(\mathrm{dd}, J=13.8,7.3,1 \mathrm{H}, \mathrm{CH}_{2}\right), 2.82 \square 2.72(\mathrm{~m}, 1 \mathrm{H}, \mathrm{CH}), 2.71 \square 2.61$ (m, 1H, CH), $2.00 \square 1.81\left(\mathrm{~m}, 2 \mathrm{H}, \mathrm{CH}_{2}\right), 1.59 \square 1.51\left(\mathrm{~m}, 4 \mathrm{H}, \mathrm{CH}_{3}, \mathrm{CH}\right), 1.07$ (ddd, J = 10.5, 6.9, 3.8 $\left.\mathrm{Hz}, 3 \mathrm{H}, \mathrm{CH}_{3}\right), 0.94\left(\mathrm{dd}, J=10.7,7.2 \mathrm{~Hz}, 3 \mathrm{H}, \mathrm{CH}_{3}\right) ;{ }^{13} \mathrm{C} \mathrm{NMR}\left(125 \mathrm{MHz}, \mathrm{CDCl}_{3}\right) \square 180.54(\mathrm{dd}$, $\left.{ }^{3} J(\mathrm{C}, \mathrm{P})=2.4,0.5 \mathrm{~Hz}\right), 180.32\left(\mathrm{~d},{ }^{4} J(\mathrm{C}, \mathrm{P})=1.8 \mathrm{~Hz}\right), 152.84,142.36,136.69,136.62(2 \mathrm{C}), 136.52$, $135.05,134.94,134.30,134.05,132.08,131.99,131.70,131.64,131.23,130.87,130.45$, 130.22 (m, 3C), 128.89ロ127.76 (m, 14C), 126.72, 124.82, 124.03, 122.24, 121.66, 119.15, 108.26, 104.25, 104.21, 65.19, 64.38, 59.73, 44.23, $42.30\left(\mathrm{dd},{ }^{2} J(\mathrm{C}, \mathrm{P})=95.3,6.3 \mathrm{~Hz}\right), 35.97$ 
(br), $29.29(\mathrm{dd}, J(\mathrm{C}, \mathrm{P})=19.9,1.4 \mathrm{~Hz}), 25.84(\mathrm{dd}, J(\mathrm{C}, \mathrm{P})=23.1,3.2 \mathrm{~Hz}), 20.15\left(\mathrm{dd},{ }^{3} J(\mathrm{C}, \mathrm{P})=7.9\right.$, $1.0 \mathrm{~Hz}), 18.62(\mathrm{dd}, J(\mathrm{C}, \mathrm{P})=4.2,2.3 \mathrm{~Hz}), 15.66(\mathrm{~d}, J(\mathrm{C}, \mathrm{P})=8.8 \mathrm{~Hz}) ;{ }^{31} \mathrm{P} \mathrm{NMR}\left(162 \mathrm{MHz}, \mathrm{CDCl}_{3}\right) \square$ $28.50\left(\mathrm{~d},{ }^{2} J(\mathrm{P}, \mathrm{P})=58.5 \mathrm{~Hz}, 1.00 \mathrm{P}\right), 9.31\left(\mathrm{~d},{ }^{2} J(\mathrm{P}, \mathrm{P})=58.4 \mathrm{~Hz}, 0.92 \mathrm{P}\right) ;[\square]_{589}=+176,[\square]_{577}=$ $+187,[\square]_{546}=+217,[\square]_{435}=+431,[\square]_{405}=+583\left(c=0.21\right.$ in $\left.\mathrm{CHCl}_{3}\right)$; IR (thin film) 2922, 2953, $1691,1652,1594,1463,1436,1378,1231,1185,1100,1054,1019,980,942,799,753,722$, $699 \mathrm{~cm}^{\square 1}$; LRMS (ESI+) Calcd for $\mathrm{C}_{56} \mathrm{H}_{53} \mathrm{~N}_{2} \mathrm{O}_{4} \mathrm{P}_{2} \mathrm{Pd}(\mathrm{M}+\mathrm{H})^{+}$: 985.25. Found: 985.27.

Reaction of 9 with Stoichiometric $\operatorname{Pd}(0) \cdot(R)$-BINAP. Following GP1, a solution of aryl triflate 2 (25 mg, $42 \square \mathrm{mol}), \mathrm{Pd}(\mathrm{OAc})_{2}(9.4 \mathrm{mg}, 42 \square \mathrm{mol}),(R)$-BINAP (52 mg, $\left.83 \square \mathrm{mol}\right)$, PMP (26 mg, $167 \square \mathrm{mol})$ and DMA $(0.5 \mathrm{~mL})$ was maintained at $80{ }^{\circ} \mathrm{C}$ for $10 \mathrm{~h}$. After the usual workup, the residue was purified by flash chromatography (50\% diethyl ether-hexanes) to afford 110 (33 mg, $28 \square \mathrm{mol}, 67 \%$, 94:6 mixture of $\square: \square$ methyl epimers) as a bright yellow powder and 12 (3.2 mg, $7 \square \mathrm{mol}, 17 \%)$ as a colorless solid:

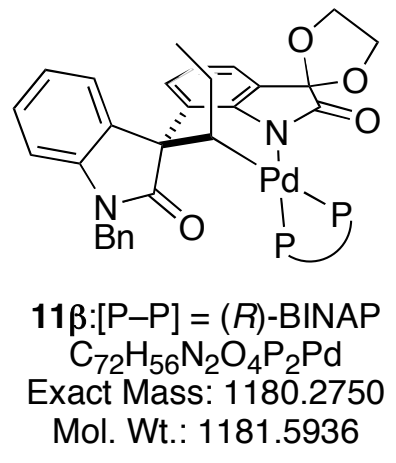

\section{$\mathrm{C}_{28} \mathrm{H}_{24} \mathrm{~N}_{2} \mathrm{O}_{4} \cdot(R)$-BINAP palladacycle 110 (characterized as a 94:6 mixture of} ethyl epimers $11 \mathrm{r}$ and $11 \mathrm{C}) . \mathrm{mp}>208{ }^{\circ} \mathrm{C}$, decomposition $\left(\mathrm{CHCl}_{3}\right) ;{ }^{1} \mathrm{H} \mathrm{NMR}\left(500 \mathrm{MHz}, \mathrm{CDCl}_{3}\right.$, peaks for major epimer) $\square 9.06(\mathrm{t}, J=8.9 \mathrm{~Hz}, 1 \mathrm{H}, \operatorname{Ar} H), 8.68(\mathrm{dd}, J=13.2,7.7 \mathrm{~Hz}, 1 \mathrm{H}, \operatorname{Ar} H)$, 7.84-7.79 (m, 4H, ArH), 7.59ロ7.54 (m, 3H, ArH), $7.49(\mathrm{~d}, J=8.2 \mathrm{~Hz}, 1 \mathrm{H}, \operatorname{Ar} H), 7.44-7.27$ (m, 10H, ArH), 7.26-7.00 (m, 8H, ArH), 6.97-6.90 (m, 4H, ArH), 6.87-6.75 (m, 3H, ArH), 6.64-6.43 $(\mathrm{m}, 6 \mathrm{H}, \operatorname{Ar} H), 6.35(\mathrm{~d}, J=8.7 \mathrm{~Hz}, 1 \mathrm{H}, \operatorname{Ar} H), 6.30(\mathrm{dd}, J=7.8,1.0 \mathrm{~Hz}, 1 \mathrm{H}, \operatorname{ArH}), 5.79(\mathrm{t}, J=7.6$ Hz, 1H, ArH), 5.38 (d, J=15.3 Hz, 1H, CH $\left.H_{2}\right), 4.97$ (d, J=15.3 Hz, 1H, $\left.\mathrm{CH}_{2}\right), 4.44(\mathrm{td}, J=7.1,3.8$ $\left.\mathrm{Hz}, 1 \mathrm{H}, \mathrm{CH}_{2}\right), 4.10\left(\mathrm{q}, J=7.4 \mathrm{~Hz}, 1 \mathrm{H}, \mathrm{CH}_{2}\right), 3.96\left(\mathrm{td}, J=7.0,3.7 \mathrm{~Hz}, 1 \mathrm{H}, \mathrm{CH}_{2}\right), 3.73(\mathrm{q}, J=7.4 \mathrm{~Hz}$, 
$\left.1 \mathrm{H}, \mathrm{CH}_{2}\right), 2.02 \square 1.89(\mathrm{~m}, 1 \mathrm{H}, \mathrm{CH}), 1.55 \square 1.34\left(\mathrm{~m}, 2 \mathrm{H}, \mathrm{CH}_{2}\right),-0.10\left(\mathrm{t}, J=7.6 \mathrm{~Hz}, 3 \mathrm{H}, \mathrm{CH}_{3}\right) ;{ }^{13} \mathrm{C}$ NMR $\left(125 \mathrm{MHz}, \mathrm{CDCl}_{3}\right) \mathrm{1} 180.90\left(\mathrm{dd},{ }^{3} J(\mathrm{C}, \mathrm{P})=3.2,0.5 \mathrm{~Hz}\right), 180.38\left(\mathrm{~d},{ }^{4} J(\mathrm{C}, \mathrm{P})=2.8 \mathrm{~Hz}\right), 153.93(\mathrm{~d}$, $J(\mathrm{C}, \mathrm{P})=1.4 \mathrm{~Hz}), 142.63,141.81(\mathrm{dd}, J(\mathrm{C}, \mathrm{P})=9.7,2.3 \mathrm{~Hz}), 137.05(\mathrm{~d}, J(\mathrm{C}, \mathrm{P})=1.9 \mathrm{~Hz}), 136.79$ $(\mathrm{d}, J(\mathrm{C}, \mathrm{P})=1.9 \mathrm{~Hz}), 136.52,136.45(\mathrm{dd}, J(\mathrm{C}, \mathrm{P})=7.9,4.2 \mathrm{~Hz}), 134.65(2 \mathrm{C}), 134.45,134.38$ $134.32(\mathrm{~d}, J(\mathrm{C}, \mathrm{P})=8.3 \mathrm{~Hz}), 134.24(\mathrm{~d}, J(\mathrm{C}, \mathrm{P})=6.0 \mathrm{~Hz}), 134.12,133.93,133.52,133.40(\mathrm{~d}$, $J(\mathrm{C}, \mathrm{P})=1.4 \mathrm{~Hz}), 132.85,132.69(\mathrm{~d}, J(\mathrm{C}, \mathrm{P})=10.2 \mathrm{~Hz}), 132.48(\mathrm{~d}, J(\mathrm{C}, \mathrm{P})=6.0 \mathrm{~Hz}), 132.21(\mathrm{~d}$, $J(\mathrm{C}, \mathrm{P})=9.3 \mathrm{~Hz}), 132.10,131.87,131.42,131.30,131.16(\mathrm{~d}, J(\mathrm{C}, \mathrm{P})=11.1 \mathrm{~Hz}), 131.11,130.89$, 130.80, 130.34, $129.53(\mathrm{~d}, J(\mathrm{C}, \mathrm{P})=1.9 \mathrm{~Hz}), 129.29,129.13(\mathrm{~d}, J(\mathrm{C}, \mathrm{P})=1.9 \mathrm{~Hz}), 129.05(2 \mathrm{C})$, $128.72(\mathrm{~d}, J(\mathrm{C}, \mathrm{P})=9.3 \mathrm{~Hz}), 128.46(\mathrm{~d}, J(\mathrm{C}, \mathrm{P})=8.3 \mathrm{~Hz}), 128.36(2 \mathrm{C}), 128.27,128.16,128.11$, 128.07, 128.01, 127.90, 127.74, 127.70, 127.37, (d, J(C,P) = 5.1 Hz), $127.28(\mathrm{~d}, J(\mathrm{C}, \mathrm{P})=4.6 \mathrm{~Hz})$, $127.10(\mathrm{~d}, J(\mathrm{C}, \mathrm{P})=1.9 \mathrm{~Hz}), 126.87,126.74,126.37,126.08,126.04,125.43,125.20,124.27$, 122.36, 122.07, 119.06, 108.51, $104.55\left(\mathrm{dd},{ }^{4} J(\mathrm{C}, \mathrm{P})=5.1,1.0 \mathrm{~Hz}\right), 65.71,64.16,60.48(\mathrm{dd}$, $\left.{ }^{3} J(\mathrm{C}, \mathrm{P})=3.7,1.4 \mathrm{~Hz}\right), 51.60\left(\mathrm{dd},{ }^{2} J(\mathrm{C}, \mathrm{P})=96.4,6.5 \mathrm{~Hz}\right), 44.40,27.29(\mathrm{~m}), 15.01\left(\mathrm{~d},{ }^{4} J(\mathrm{C}, \mathrm{P})=2.3\right.$ $\mathrm{Hz}) ;{ }^{31} \mathrm{P}$ NMR (2O2 MHz, $\left.\mathrm{CDCl}_{3}\right) \square 28.3\left(\mathrm{~d},{ }^{2} J(\mathrm{P}, \mathrm{P})=52.2 \mathrm{~Hz}, 0.97 \mathrm{P}\right), 27.3\left(\mathrm{~d},{ }^{2} J(\mathrm{P}, \mathrm{P})=50.9 \mathrm{~Hz}\right.$, 0.05P), $17.6\left(\mathrm{~d},{ }^{2} J(\mathrm{P}, \mathrm{P})=52.2 \mathrm{~Hz}, 0.95 \mathrm{P}\right), 16.2\left(\mathrm{~d},{ }^{2} J(\mathrm{P}, \mathrm{P})=50.9 \mathrm{~Hz}, 0.05 \mathrm{P}\right) ;[\square]_{589}=+190,[\square]_{577}$ $=+209,[\square]_{546}=+278,[\square]_{435}$ and $[\square]_{405}$ not possible due to sample absorbance $\left(c=3.1\right.$ in $\left.\mathrm{CHCl}_{3}\right)$; IR (thin film) 3059, 2958, 2927, 2866, 1692, 1677, 1607, 1483, 1437, 1344, 1298, 1182, 1128 , 1004, 742, $695 \mathrm{~cm}^{\square 1}$; LRMS (ESI) Calcd for $\mathrm{C}_{72} \mathrm{H}_{57} \mathrm{~N}_{2} \mathrm{O}_{4} \mathrm{P}_{2} \mathrm{Pd}(\mathrm{M}+\mathrm{H})^{+}:$1181.3. Found: 1181.3.

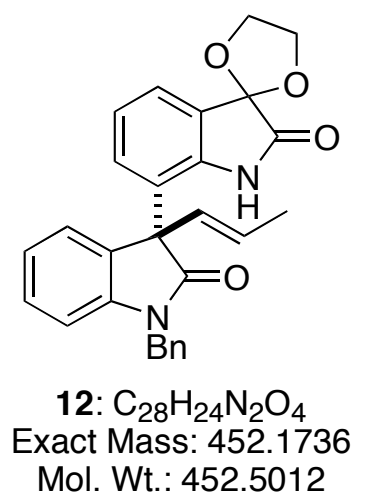


(S)-1-Benzyl-3-prop-1-enyl-1,3-dihydro-3,3'-spiro[1,3-dioxolane]-1'H-

[3,7']biindoyl-2,2'-dione (12): mp 87-89 ${ }^{\circ} \mathrm{C}$ (diethyl ether/hexanes); HPLC ${ }^{4}$ (Daicel Chiracel OD-H column, column temperature $45{ }^{\circ} \mathrm{C}, n$-hexane-2-propanol $=96: 4$, flow rate $=0.5 \mathrm{~mL} \cdot \mathrm{min}^{-}$

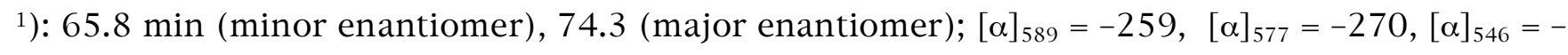
313, $[\square]_{435}=-592,[\square]_{405}=-788\left(c=1.4\right.$ in $\left.\mathrm{CHCl}_{3}\right) ;{ }^{1} \mathrm{H}$ NMR $\left(500 \mathrm{MHz}, \mathrm{CDCl}_{3}\right) \square 8.63(\mathrm{br} \mathrm{s}, 1 \mathrm{H}$, NH), 7.32-7.20 (m, 8H, ArH), 7.17-7.12 (m, 1H, ArH), $7.00(\mathrm{dd}, J=8.3,1.6 \mathrm{~Hz}, 1 \mathrm{H}, \operatorname{Ar} H), 6.93$ $(\mathrm{t}, J=7.8 \mathrm{~Hz}, 1 \mathrm{H}, \operatorname{Ar} H), 6.83-6.79(\mathrm{~m}, 1 \mathrm{H}, \operatorname{Ar} H), 5.79(\mathrm{dq}, J=15.4,1.5 \mathrm{~Hz}, 1 \mathrm{H}, \mathrm{CH}), 5.63(\mathrm{dq}, J=$ 15.4, 6.4 Hz, 1H, CH), $4.91\left(\mathrm{~s}, 2 \mathrm{H}, \mathrm{CH}_{2}\right), 4.64 \square 4.54\left(\mathrm{~m}, 2 \mathrm{H}, \mathrm{CH}_{2}\right), 4.36 \square 4.29\left(\mathrm{~m}, 2 \mathrm{H}, \mathrm{CH}_{2}\right), 1.74$ $\left(\mathrm{dd}, J=6.5,1.6 \mathrm{~Hz}, 3 \mathrm{H}, \mathrm{CH}_{3}\right) ;{ }^{13} \mathrm{C} \mathrm{NMR}\left(125 \mathrm{MHz}, \mathrm{CDCl}_{3}\right) \square 177.32,174.40,142.47,142.19$, 135.49, 130.93, 130.28, 129.16, 129.11 (2C), 128.98, 128.71, 127.99, 127.33 (2C), 126.49, 126.22, 124.84, 123.24. 123.10, 121.26, 110.30, 101.77, 66.06, 65.82, 58.53, 44.21, 18.22; IR (thin film) 2976, 2899, 2810, 1741, 1698, 1610, 1486, 1467, 1444, 1366, 1316, 1177, 1139, 1007, 946, 753, 699, $664 \mathrm{~cm}^{\square 1}$; HRMS (CI+) Calcd for $\mathrm{C}_{28} \mathrm{H}_{25} \mathrm{~N}_{2} \mathrm{O}_{4}(\mathrm{M}+\mathrm{H})^{+}$: 453.1814. Found: 453.1814.

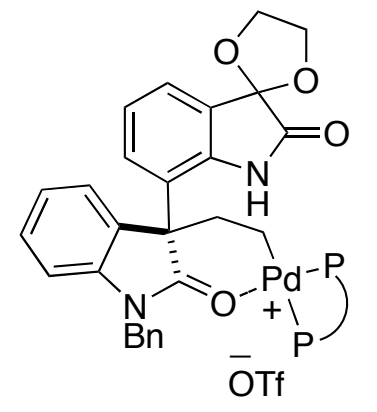

$$
\begin{aligned}
& \text { 13: }[\mathrm{P}-\mathrm{P}]=(R)-\mathrm{BINAP} \\
& \mathrm{C}_{71} \mathrm{H}_{55} \mathrm{~N}_{2} \mathrm{O}_{4} \mathrm{P}_{2} \mathrm{Pd}^{+} \\
& \text {Exact Mass: } 1167.2666 \\
& \text { Mol. Wt.: } 1168.5744
\end{aligned}
$$

Cationic $(R)$-BINAP palladacycle (13). A solution of 2,6-di-tert-butyl-pyridine hydrotriflate $(24 \mathrm{mg}, 69 \square \mathrm{mol})$ and degassed THF-d8 $(0.2 \mathrm{~mL})$ was added to a solution of $3 \square$ (35

\footnotetext{
${ }^{4}$ HPLC analysis to determine enantiomeric purity was calibrated with a sample of the corresponding racemate.
} 
$\mathrm{mg}, 30 \square \mathrm{mol})$ in degassed THF $(0.5 \mathrm{~mL})$ in an NMR tube. After $<3 \mathrm{~min}$, quantitative conversion to 13 was confirmed by ${ }^{31} \mathrm{P}$ and ${ }^{1} \mathrm{H}$ NMR: ${ }^{1} \mathrm{H}$ NMR $\left(500 \mathrm{MHz}, \mathrm{CDCl}_{3}\right) \square 8.70 \square 8.40($ br s, $1 \mathrm{H}, \mathrm{NH})$, 7.97๑6.72 (m, 43H, ArH), $6.56(\mathrm{~d}, J=8.7 \mathrm{~Hz}, 1 \mathrm{H}, \operatorname{Ar} H), 5.00(\mathrm{~d}, J=15.4 \mathrm{~Hz}, 1 \mathrm{H}, \mathrm{CH}), 4.64(\mathrm{~d}, J$ $\left.=15.5 \mathrm{~Hz}, 1 \mathrm{H}, \mathrm{CH}_{2}\right), 4.67 \square 4.38\left(\mathrm{~m}, 2 \mathrm{H}, \mathrm{CH}_{2}\right), 4.27 \square 4.19\left(\mathrm{~m}, 2 \mathrm{H}, \mathrm{CH}_{2}\right), 2.37 \square 2.21\left(\mathrm{br} \mathrm{m}, 1 \mathrm{H}, \mathrm{CH}_{2}\right)$, $1.95 \square 1.93\left(\mathrm{~m}, 1 \mathrm{H}, \mathrm{CH}_{2}\right), 1.09 \square 1.04\left(\mathrm{~m}, 1 \mathrm{H}, \mathrm{CH}_{2}\right)$, one methylene hydrogen is obscured; ${ }^{13} \mathrm{C}$ NMR (125 MHz, THF-d8) \ 184.89 (br m), 175.62, 144.25, 143.60, 141.98, 141.16, 140.69 (br), $139.60,139.55,139.51,139.45,139.42,137.28,136.86$ (br), 136.38, 135.89, 135.72, 135.54, 135.44, $135.21(\mathrm{~d}, J(\mathrm{C}, \mathrm{P})=2.3 \mathrm{~Hz}), 134.85,134.74,134.51,134.44,134.08,134.02,133.26$, 131.92-129.14 (m, 10C), 128.99, 128.80, 128.76, 128.71, 128.51, 128.47, 128.37. 128.28, 128.11, 127.94 (br), 127.84, 127.79, 127.56, 127.43, 127.16, 126.35, 126.22, 125.94, 125.62, $125.43,124.18,124.14,123.66,122.78,121.10,112.77,111.30,111.20,101.58,66.62,66.56$, $66.47\left(\mathrm{dd},{ }^{4} J(\mathrm{C}, \mathrm{P})=45.8,3.7 \mathrm{~Hz}\right), 46.30(\mathrm{br}), 37.27(\mathrm{br}), 34.88\left(\mathrm{dd},{ }^{2} J(\mathrm{C}, \mathrm{P})=88.3,7.4 \mathrm{~Hz}\right) ;{ }^{31} \mathrm{P}$ NMR $\left(202 \mathrm{MHz}, \mathrm{THF}_{\mathrm{d} 8}\right) \square 41.9\left(\mathrm{~d},{ }^{2} J(\mathrm{P}, \mathrm{P})=56.8 \mathrm{~Hz}, 1.00 \mathrm{P}\right), 15.4\left(\mathrm{~d},{ }^{2} J(\mathrm{P}, \mathrm{P})=49.5 \mathrm{~Hz}, 1.07 \mathrm{P}\right)$; LRMS (ESI+) Calcd for $\mathrm{C}_{71} \mathrm{H}_{55} \mathrm{~N}_{2} \mathrm{O}_{4} \mathrm{P}_{2} \mathrm{Pd}\left(\mathrm{M}^{+}\right)$: 1167.3. Found: 1167.2.

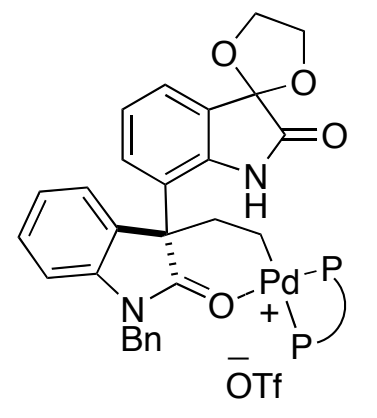

14: $[\mathrm{P}-\mathrm{P}]=(R, R)-\mathrm{BDPP}$

$\mathrm{C}_{56} \mathrm{H}_{53} \mathrm{~N}_{2} \mathrm{O}_{4} \mathrm{P}_{2} \mathrm{Pd}^{+}$

Exact Mass: 985.2510

Mol. Wt.: 986.3980

Cationic $(R, R)$-BDPP palladacycle (14). A solution of 2,6-di-tert-butyl-pyridine hydrotriflate (26 mg, $76 \square \mathrm{mol})$ and degassed THF-d8 (0.2mL) was added to a solution of $10 \square$ (25 mg, $25 \square \mathrm{mol}$ ) in degassed THF $(0.6 \mathrm{~mL})$ in an NMR tube. After $<45$ sec, quantitative conversion to 14 was confirmed by ${ }^{31} \mathrm{P}$ and ${ }^{1} \mathrm{H}$ NMR: ${ }^{1} \mathrm{H}$ NMR (500 MHz, THF-d8) $\square 9.27$ (br s, 1H, NH), $7.97 \square 6.85(\mathrm{~m}, 31 \mathrm{H}, \operatorname{Ar} H), 6.34(\mathrm{~d}, J=7.6 \mathrm{~Hz}, 1 \mathrm{H}, \operatorname{Ar} H), 4.88\left(\mathrm{~d}, J=15.6 \mathrm{~Hz}, 1 \mathrm{H}, \mathrm{C} H_{2}\right)$, 
$4.83\left(\mathrm{~d}, J=15.6 \mathrm{~Hz}, 1 \mathrm{H}, \mathrm{CH}_{2}\right), 4.51 \square 4.41\left(\mathrm{~m}, 2 \mathrm{H}, \mathrm{CH}_{2}\right), 4.40 \square 4.16\left(\mathrm{~m}, 2 \mathrm{H}, \mathrm{CH}_{2}\right), 3.14 \square 2.61(\mathrm{~m}$, 2H, CH), $1.98 \square 1.84\left(\mathrm{~m}, 3 \mathrm{H}, \mathrm{CH}_{2}\right), 1.79 \square 1.72\left(\mathrm{~m}, 1 \mathrm{H}, \mathrm{CH}_{2}\right), 1.23 \square 1.14\left(\mathrm{~m}, 3 \mathrm{H}, \mathrm{CH}_{3}\right), 1.10 \square 1.02(\mathrm{~m}$, $\left.3 \mathrm{H}, \mathrm{CH}_{3}\right)$, two methylene protons of the ligand are obscured; ${ }^{13} \mathrm{C} \mathrm{NMR}(125 \mathrm{MHz}$, THF-d8) 185.12 (br m), 175.82, 167.73 (br), 148.86 (br), 142.20, 141.39, 136.69 (d, J(C,P) = 12.0 Hz), 136.11, $135.80(\mathrm{~d}, J(\mathrm{C}, \mathrm{P})=10.6 \mathrm{~Hz}), 135.62,135.15,(\mathrm{~d}, J(\mathrm{C}, \mathrm{P})=9.7 \mathrm{~Hz}), 133.79(\mathrm{~d}, J(\mathrm{C}, \mathrm{P})=$ $10.6 \mathrm{~Hz}), 132.31,132.04(\mathrm{~d}, J(\mathrm{C}, \mathrm{P})=2.3 \mathrm{~Hz}), 131.96,131.72,131.37(\mathrm{~d}, J(\mathrm{C}, \mathrm{P})=2.3 \mathrm{~Hz})$, 130.25, 130.12, 130.03, 129.90, $130.08(\mathrm{~d}, J(\mathrm{C}, \mathrm{P})=11.1 \mathrm{~Hz}), 128.70,130.35-129.26(\mathrm{~m}, 8 \mathrm{C})$, 128.95, 128.29 (br), $127.46(\mathrm{~m}, 2 \mathrm{C}), 126.10(\mathrm{~d}, J(\mathrm{C}, \mathrm{P})=13.4 \mathrm{~Hz}), 124.35(\mathrm{~d}, J(\mathrm{C}, \mathrm{P})=8.8 \mathrm{~Hz})$, 123.86-122.70 (m, 4C), 122.23, 121.11, 112.65, 101.61, 66.79, 66.69, 61.53 (d, ${ }^{4} J(\mathrm{C}, \mathrm{P})=2.3$ Hz), $45.76(\mathrm{br}), 36.36\left(\mathrm{dd},{ }^{2} J(\mathrm{C}, \mathrm{P})=9.7,1.8 \mathrm{~Hz}\right), 33.85($ br m), 30.78, $28.19(\mathrm{dd}, J(\mathrm{C}, \mathrm{P})=31.9$, $8.8 \mathrm{~Hz}), 18.09(\mathrm{br}), 17.67(\mathrm{~d}, J(\mathrm{C}, \mathrm{P})=2.3 \mathrm{~Hz}), 15.85$; ${ }^{31} \mathrm{P}$ NMR (202 MHz, THF-d8) $446.3(\mathrm{~d}$, $\left.{ }^{2} J(\mathrm{P}, \mathrm{P})=59.2 \mathrm{~Hz}, 1.00 \mathrm{P}\right), 10.6\left(\mathrm{~d},{ }^{2} J(\mathrm{P}, \mathrm{P})=59.4 \mathrm{~Hz}, 1.04 \mathrm{P}\right)$; IR (thin film) 2972, 2907, 2980, $1741 / 1735,1640,1617,1577,1532,1486,1468,1440,1413,1374,1251,1224,1154,1100$, 1031, 946, 888, 818, 749, $699 \mathrm{~cm}^{\square 1}$; LRMS (ESI+) Calcd for $\mathrm{C}_{56} \mathrm{H}_{53} \mathrm{~N}_{2} \mathrm{O}_{4} \mathrm{P}_{2} \mathrm{Pd}\left(\mathrm{M}^{+}\right)$: 985.25. Found: 985.18.

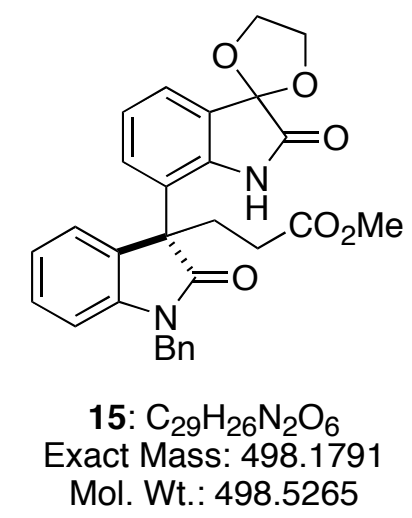

\section{3-(1-Benzyl-2,2'-dioxo-1,2,2',3'-tetrahydro-1'H-[3,7']biindoyl-3-yl-propionic}

acid methyl ester (15). 2,6-Di-tert-butylpyridine hydrotriflate (7.3 mg, $21 \square \mathrm{mol})$ was added in one portion to a solution of $3 \square(25 \mathrm{mg}, 21 \square \mathrm{mol})$ in $\mathrm{MeOH}(10 \mathrm{~mL}$, saturated with $\mathrm{CO})$. The solution was maintained for $16 \mathrm{~h}$ and poured into a saturated aqueous solution of $\mathrm{NaHCO}_{3}(10$ $\mathrm{mL})$. The organic later was separated, and the aqueous layer was extracted with $\mathrm{CH}_{2} \mathrm{Cl}_{2}(45$ 
$\mathrm{mL})$. The combined organic layers were dried $\left(\mathrm{Na}_{2} \mathrm{SO}_{4}\right)$ and concentrated. The residue was purified by chromatography (50\% diethyl ether-hexanes) to afford 15 (10 mg, 21 घmol, 99\%) as a colorless solid: mp: $91-93^{\circ} \mathrm{C}$ (diethyl ether/hexanes); ${ }^{1} \mathrm{H}$ NMR (500 MHz, $\left.\mathrm{CDCl}_{3}\right) \square 9.59$ (br s, 1H, NH), $7.36(\mathrm{~d}, J=7.5 \mathrm{~Hz}, 1 \mathrm{H}, \operatorname{Ar} H), 7.33-7.18(\mathrm{~m}, 8 \mathrm{H}, \operatorname{Ar} H), 7.10(\mathrm{dd}, J=8.0,1.1 \mathrm{~Hz}, 1 \mathrm{H}$, $\operatorname{Ar} H), 6.94(\mathrm{dd}, J=7.7 \mathrm{~Hz}, 1 \mathrm{H}, \operatorname{Ar} H), 6.86(\mathrm{~d}, J=7.7 \mathrm{~Hz}, 1 \mathrm{H}, \operatorname{Ar} H), 4.92\left(\mathrm{~d}, J=15.7 \mathrm{~Hz}, 1 \mathrm{H}, \mathrm{C} H_{2}\right)$, $4.87\left(\mathrm{~d}, J=15.7 \mathrm{~Hz}, 1 \mathrm{H}, \mathrm{CH}_{2}\right), 4.65-4.55\left(\mathrm{~m}, 2 \mathrm{H}, \mathrm{CH}_{2}\right), 4.36 \square 4.29(\mathrm{~m}, 2 \mathrm{H}, \mathrm{CH}), 3.56(\mathrm{~s}, 3 \mathrm{H}$, $\left.\mathrm{OCH}_{3}\right), 2.91\left(\mathrm{ddd}, J=14.1,11.1,5.1 \mathrm{~Hz}, 1 \mathrm{H}, \mathrm{CH}_{2}\right), 2.83\left(\mathrm{ddd}, J=14.1,11.4,5.1 \mathrm{~Hz}, 1 \mathrm{H}, \mathrm{CH}_{2}\right)$, $2.40\left(\mathrm{ddd}, J=15.9,11.2,5.0 \mathrm{~Hz}, 1 \mathrm{H}, \mathrm{CH}_{2}\right), 2.18\left(\mathrm{ddd}, J=15.9,11.2,5.1 \mathrm{~Hz}, 1 \mathrm{H}, \mathrm{CH}_{2}\right) ;{ }^{13} \mathrm{C}$ NMR $\left(125 \mathrm{MHz}, \mathrm{CDCl}_{3}\right) \square 178.30,175.00,172.56,142.98,142.32,135.63,135.29,130.67,129.62$, 129.51, 129.18 (2C), 129.15, 128.14, 127.48 (2C), 126.50, 125.00, 123.33, 123.27, 121.04, 110.52, 101.63, 66.12, 65.81, 56.07, 52.00, 44.50, 31.20; IR (thin film) 3065, 3034, 2972, 2953, 2856, 1741, 1691, 1606, 1486, 1467, 1440, 1370, 1312, 1177, 1135, 1027, 1007, $753 \mathrm{~cm}^{\square 1}$; HRMS (CI+) Calcd for $\mathrm{C}_{29} \mathrm{H}_{26} \mathrm{~N}_{2} \mathrm{O}_{6} \mathrm{Na}(\mathrm{M}+\mathrm{Na})^{+}:$521.1689. Found: 521.1674.

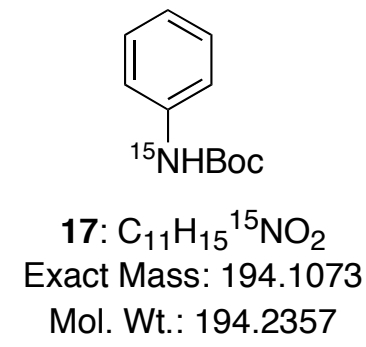

${ }^{15} N$-Phenyl carbamic acid tert-butyl ester (17). HRMS (EI+) Calcd for $\mathrm{C}_{11} \mathrm{H}_{15}{ }^{15} \mathrm{NO}_{2}(\mathrm{M})^{+}:$194.1073. Found: 194.1077 .

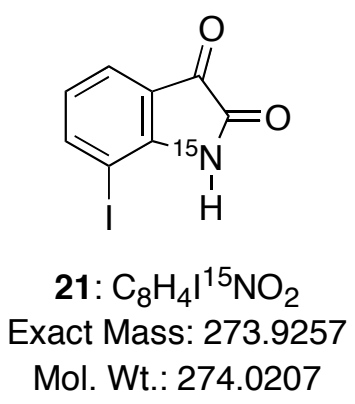

${ }^{15} \mathrm{~N}$-7-Iodo-1 $H$-indole-2,3-dione (21). HRMS (EI+) Calcd for $\mathrm{C}_{8} \mathrm{H}_{4} \mathrm{I}^{15} \mathrm{NO}_{2}(\mathrm{M})^{+}$: 273.9257. Found: 273.9263. 
<smiles>O=C1Nc2c(I)cccc2C12OCCO2</smiles>

\section{$\left[{ }^{15} \mathrm{~N}\right] 8: \mathrm{C}_{10} \mathrm{H}_{8} \mathrm{I}^{15} \mathrm{NO}_{3}$ \\ Exact Mass: 317.9519 \\ Mol. Wt.: 318.0733}

${ }^{15} \mathrm{~N}$-3,3-spiro[1,3-dioxolane]-7-iodo- $1 \mathrm{H}$-indole-2-one ([15N]8). HRMS (EI+) Calcd for $\mathrm{C}_{10} \mathrm{H}_{8} \mathrm{I}^{15} \mathrm{NO}_{3}(\mathrm{M})^{+}: 317.9519$. Found: 317.9528 .

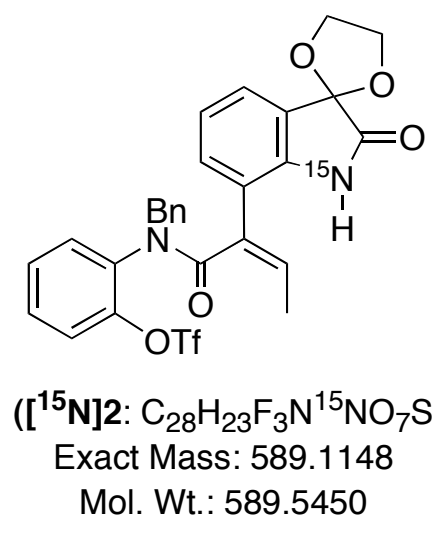

Trifluoromethanesulfonic acid (Z)-2-\{benzyl-[2-(2-oxo-3,3-spiro[1,3dioxolane]-2,3-dihydro-1 $H$-indol-7-yl)-but-2-enoyl]- $\quad{ }^{15} \mathrm{~N}$-amino\}-phenyl ester ([ $\left.\left.{ }^{15} \mathrm{~N}\right] 2\right)$. HRMS (ESI+) Calcd for $\mathrm{C}_{28} \mathrm{H}_{23} \mathrm{~F}_{3}{ }^{15} \mathrm{NNO}_{7} \mathrm{SNa}(\mathrm{M}+\mathrm{Na})^{+}:$612.1046. Found: 612.1044.<smiles>[Te]=c1[nH]c2ccccc2o1</smiles>

24: $\mathrm{C}_{7} \mathrm{H}_{5} \mathrm{NO}$ Exact Mass: 119.0371

Mol. Wt.: 119.1207

$3 H$-Benzooxazol-2-one $\left(65 \%{ }^{17} \mathrm{O}\right)$ (24). HRMS (ESI+) Calcd for $\mathrm{C}_{7} \mathrm{H}_{5} \mathrm{~N}^{17} \mathrm{OO}(\mathrm{M})^{+}$: 136.0363. Found: 136.0366.<smiles>[O+]=c1oc2ccccc2n1Cc1ccccc1</smiles>

$\left[{ }^{17} \mathrm{O}\right] 5: \mathrm{C}_{14} \mathrm{H}_{11} \mathrm{NO}$ Exact Mass: 209.0841

Mol. Wt.: 209.2432 
3-Benzyl-3 H-Benzooxazol-2-one $\left(65 \%{ }^{17} \mathrm{O}\right) \quad\left(\left[{ }^{17} \mathrm{O}\right] 5\right)$. HRMS (EI+) Calcd for $\mathrm{C}_{14} \mathrm{H}_{11} \mathrm{~N}^{17} \mathrm{OO}(\mathrm{M})^{+}:$226.0832. Found: 226.0829 .

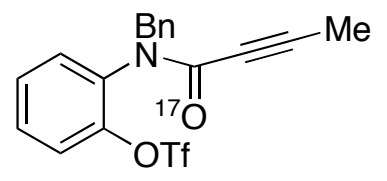

25: $\mathrm{C}_{18} \mathrm{H}_{14} \mathrm{~F}_{3} \mathrm{NO}_{3}{ }^{17} \mathrm{OS}$

Exact Mass: 398.0638

Mol. Wt.: 398.3680

Trifluoromethanesulfonic acid 2-[benzyl-(1-170-oxo-but-2-ynyl)-amino]phenyl ester $\left(65 \%{ }^{17} \mathrm{O}\right)(25)$. HRMS (ESI+) Calcd for $\mathrm{C}_{18} \mathrm{H}_{14} \mathrm{~F}_{3} \mathrm{~N}^{17} \mathrm{OO}_{3} \mathrm{~S}(\mathrm{M}+\mathrm{Na})^{+}: 421.0535$. Found: 421.0540.

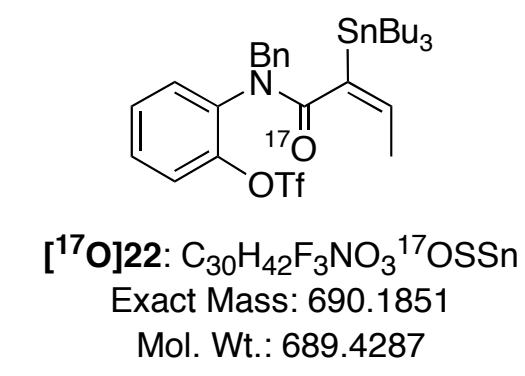

Trifluoromethanesulfonic acid (E)-2-\{benzyl-[2-(tributyl-stannanyl)-but-2${ }^{17} \mathrm{O}$-enoyl]-amino\}-phenyl ester $\left(65 \%{ }^{17} \mathrm{O}\right) \quad\left(\left[{ }^{17} \mathrm{O}\right] 22\right)$. HRMS (ESI+) Calcd for $\mathrm{C}_{30} \mathrm{H}_{42} \mathrm{~F}_{3} \mathrm{~N}^{17} \mathrm{OO}_{3} \mathrm{~S}^{116} \mathrm{Sn}(\mathrm{M})^{+}:$687.1925. Found: 687.1923.

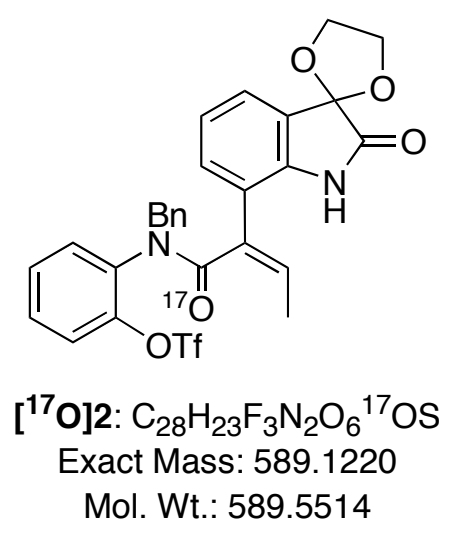


Trifluoromethanesulfonic acid (Z)-2-\{benzyl-[2-(2-oxo-3,3-spiro[1,3dioxolane]-2,3-dihydro-1 $H$-indol-7-yl)-but-2-en-17O-oyl]-amino\}-phenyl ester ([170]2). HRMS (ESI+) Calcd for $\mathrm{C}_{28} \mathrm{H}_{23} \mathrm{~F}_{3} \mathrm{~N}_{2}$ 170O ${ }_{6} \mathrm{SNa}(\mathrm{M}+\mathrm{Na})^{+}:$612.1118. Found: 612.1104.
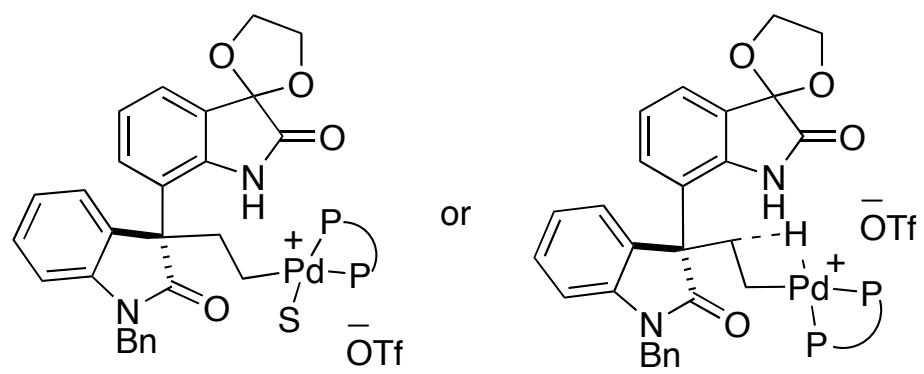

27: $[\mathrm{P}-\mathrm{P}]=(R)-\mathrm{BINAP}, \mathrm{S}=\mathrm{THF}$

$\mathrm{C}_{71} \mathrm{H}_{55} \mathrm{~N}_{2} \mathrm{O}_{4} \mathrm{P}_{2} \mathrm{Pd}^{+}$

Exact Mass: 1167.2666

Mol. Wt.: 1168.5744

Cationic $\quad \operatorname{Pd}(\mathrm{II}) \cdot(R)$-BINAP Palladacycle $\quad(27) . \quad$ 2,6-Di-tert-butyl-pyridine hydrotriflate $(3.2 \mathrm{mg}, 9.4 \square \mathrm{mol})$ was added to a solution of $13(11 \mathrm{mg}, 9.4 \square \mathrm{mol})$ and degassed THF-d8 (0.45 mL) at $-60{ }^{\circ} \mathrm{C}$ in an NMR tube. The solution was warmed to $\sim-40$ to $-35{ }^{\circ} \mathrm{C}$ at which time conversion to 27 as the major product was confirmed by ${ }^{31} \mathrm{P}\left\{{ }^{1} \mathrm{H}\right\}$ NMR: ${ }^{1} \mathrm{H}$ NMR $(500$ $\left.\mathrm{MHz}, \mathrm{THF}-\mathrm{d} 8,-35^{\circ} \mathrm{C}\right) \square 9.03(\mathrm{br} \mathrm{s}, 1 \mathrm{H}, \mathrm{NH}), 8.15-6.47(\mathrm{~m}, 44 \mathrm{H}, \operatorname{Ar} H), 5.01(\mathrm{~d}, J=16.2 \mathrm{~Hz}, 1 \mathrm{H}$, $\left.\mathrm{CH}_{2}\right), 4.66\left(\mathrm{~d}, \mathrm{~J}=15.4 \mathrm{~Hz}, 1 \mathrm{H}, \mathrm{CH}_{2}\right), 4.54-4.34\left(\mathrm{~m}, 2 \mathrm{H}, \mathrm{CH}_{2}\right), 4.31 \square 4.15\left(\mathrm{~m}, 2 \mathrm{H}, \mathrm{CH}_{2}\right), 2.72-2.47$ $\left(\mathrm{m}, 1 \mathrm{H}, \mathrm{CH}_{2}\right), 2.46-2.28\left(\mathrm{~m}, 1 \mathrm{H}, \mathrm{CH}_{2}\right), 2.04-1.86\left(\mathrm{~m}, 1 \mathrm{H}, \mathrm{CH}_{2}\right)$, one methylene proton obscured by THF solvent peak; cross-peak in COSY NMR spectrum indicates methylene multiplet is centered at $\sim 1.8 \mathrm{ppm}] ;{ }^{13} \mathrm{C}$ NMR $\left(125 \mathrm{MHz}\right.$, THF-d8, $\left.-35{ }^{\circ} \mathrm{C}\right) \mathrm{l} 179.04,175.51,167.94,165.27$, 149.03, 143.36-115.20 (58C), 110.66, 102.50, two ketal methylene carbons obscured by THF solvent peak, $59.09\left(\mathrm{dd},{ }^{4} J(\mathrm{C}, \mathrm{P})=16.7,3.2 \mathrm{~Hz}\right), 43.62(\mathrm{br} \mathrm{m}), 35.61$ (br m), $33.48(\mathrm{br} \mathrm{m}) ;{ }^{31} \mathrm{P}$ NMR $\left(162 \mathrm{MHz}, \mathrm{THF}-\mathrm{d} 8,-35^{\circ} \mathrm{C}\right) \square 43.4\left(\mathrm{~d},{ }^{2} J(\mathrm{P}, \mathrm{P})=50.5 \mathrm{~Hz}, 1.00 \mathrm{P}\right), 17.7\left(\mathrm{~d},{ }^{2} J(\mathrm{P}, \mathrm{P})=49.5 \mathrm{~Hz}\right.$, 1.04P); LRMS (ESI+) Calcd for $\mathrm{C}_{71} \mathrm{H}_{55} \mathrm{~N}_{2} \mathrm{O}_{4} \mathrm{P}_{2} \mathrm{Pd}\left(\mathrm{M}^{+}\right)$: 1167.3. Found: 1167.3 . 

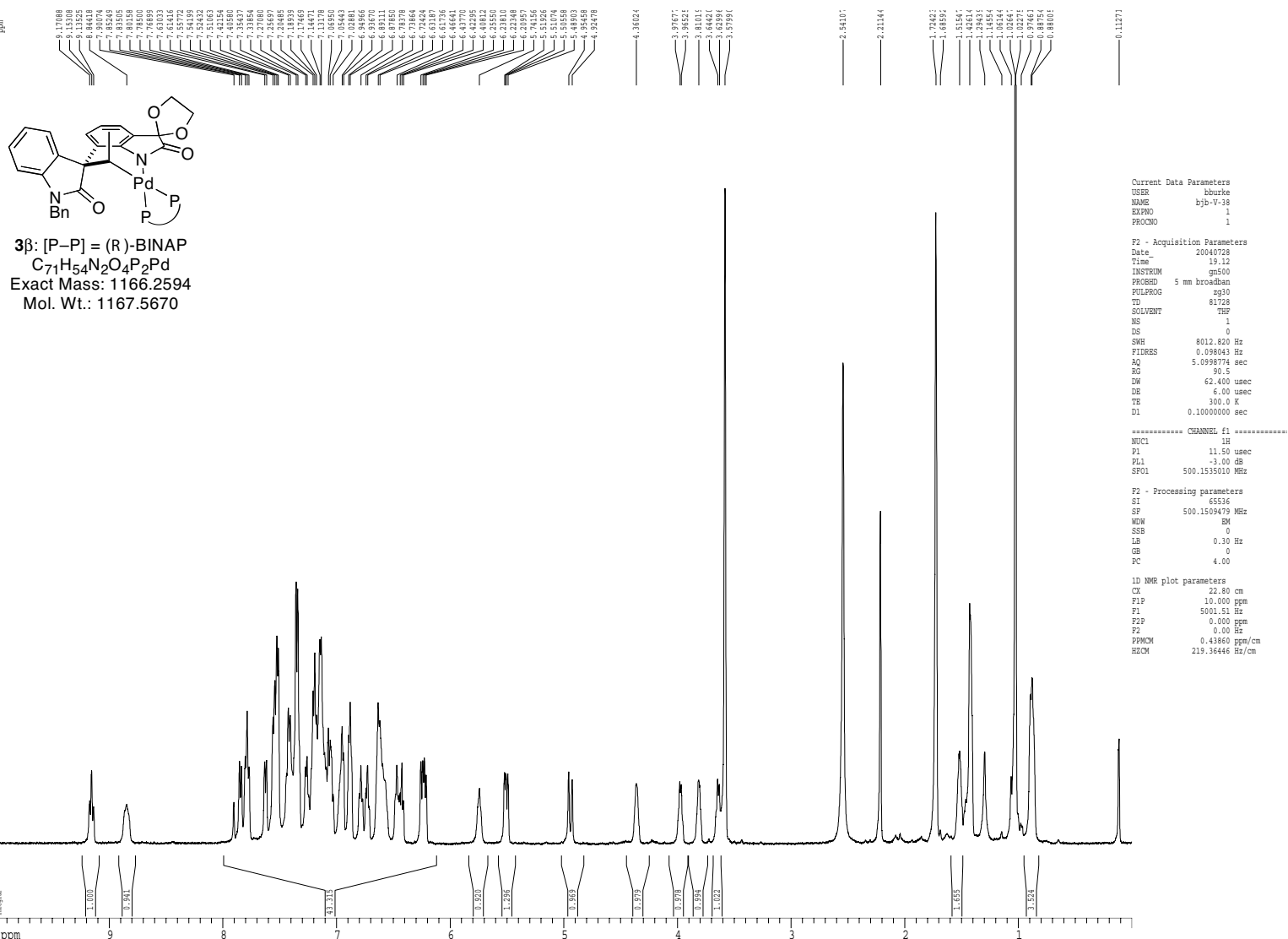

33: $[\mathrm{P}-\mathrm{P}]=(\mathrm{R})-\mathrm{BINAP}$

$\mathrm{C}_{71} \mathrm{H}_{54} \mathrm{~N}_{2} \mathrm{O}_{4} \mathrm{P}_{2} \mathrm{Pd}$

xact Mass. 1166.2594

Mol. Wt.: 1167.5670
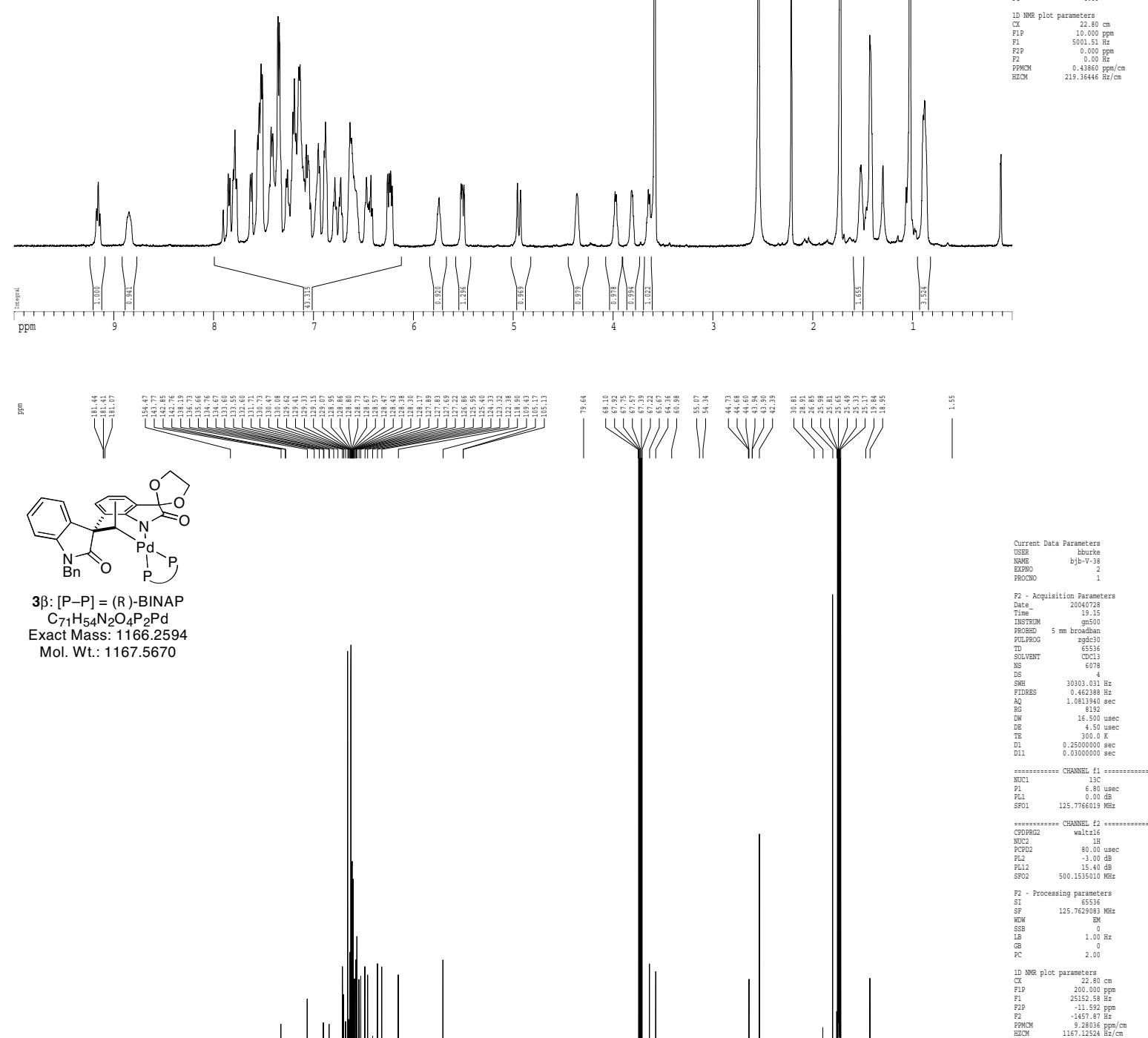

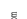$$
\text { 琶新 }
$$

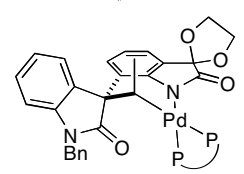

3B: $[\mathrm{P}-\mathrm{P}]=(\mathrm{R})-\mathrm{BINAP}$ $\mathrm{C}_{71} \mathrm{H}_{54} \mathrm{~N}_{2} \mathrm{O}_{4} \mathrm{P}_{2} \mathrm{Pd}$ Exact Mass: 1166.2594
Mol. Wt.: 1167.5670

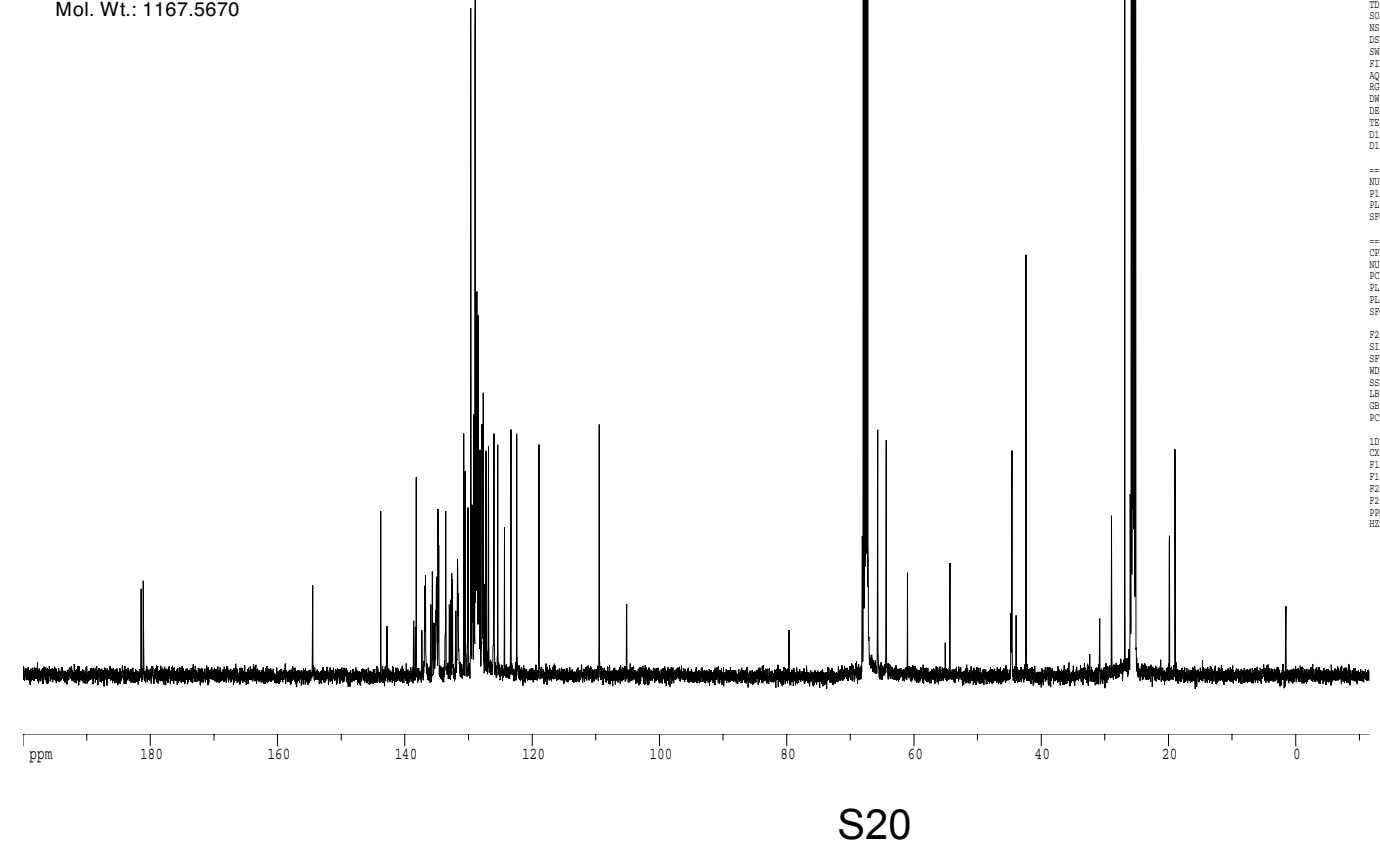




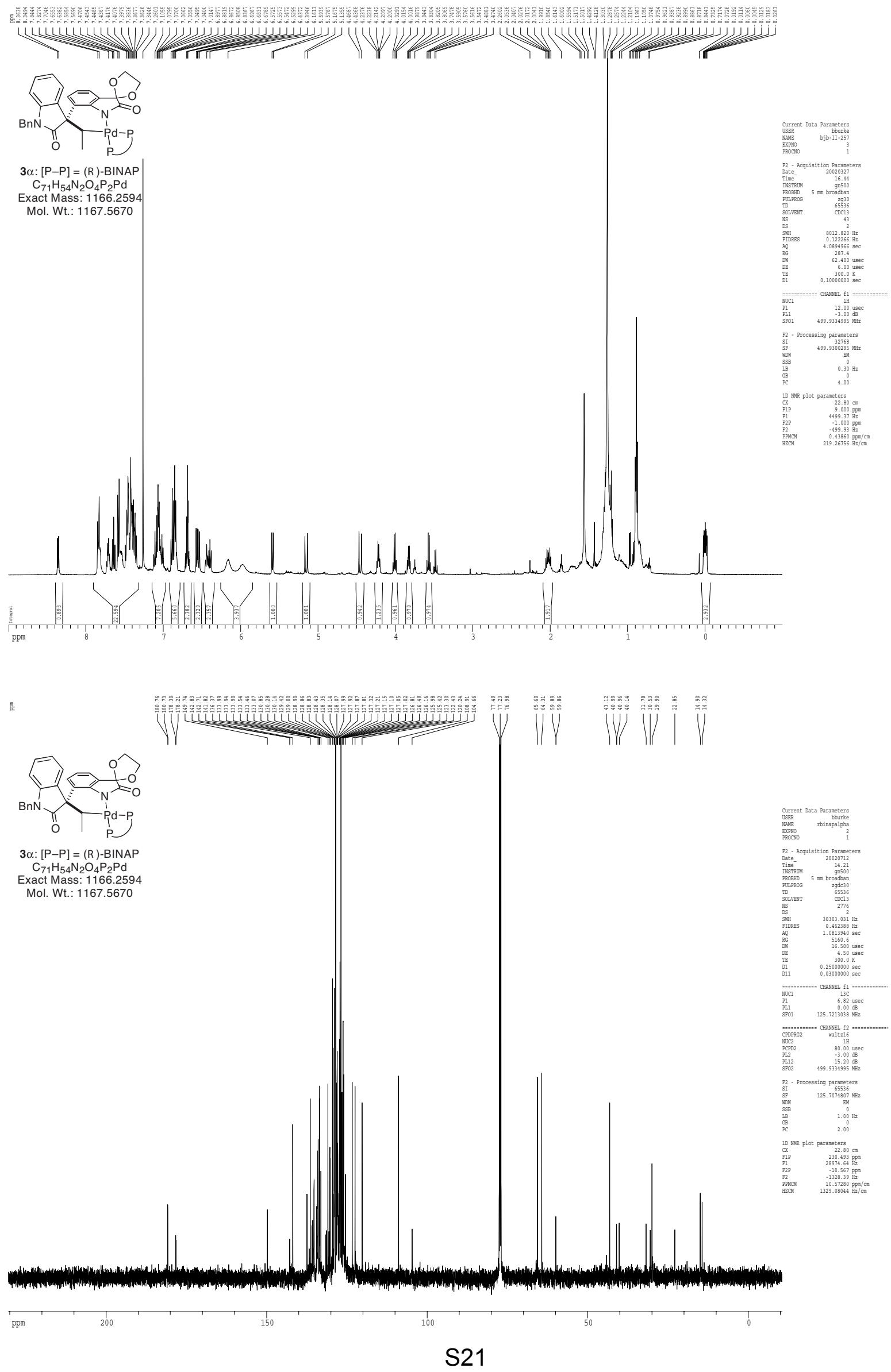




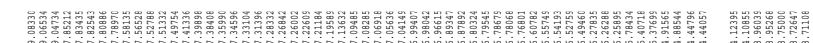

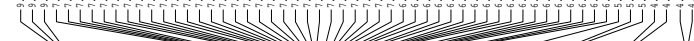

UV
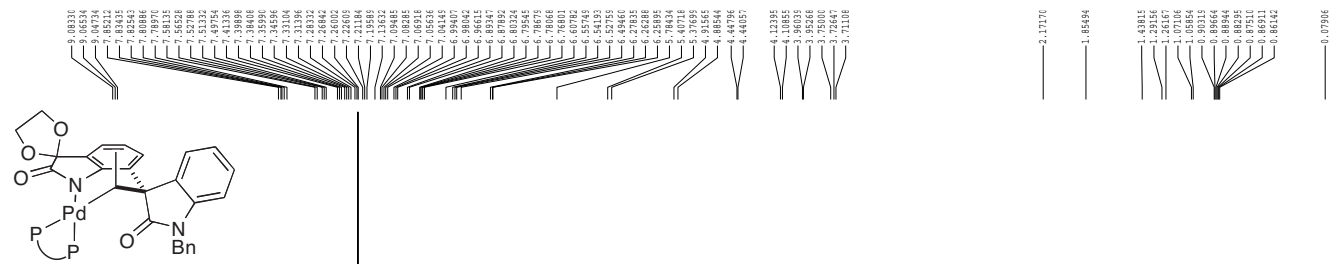

ent-3B: $[P-P]=(S)$-BINAP

$\mathrm{C}_{71} \mathrm{H}_{54} \mathrm{~N}_{2} \mathrm{O}_{4} \mathrm{P}_{2} \mathrm{Pd}$

Exact Mass: 1166.2594

Mol. Wt.: 1167.5670

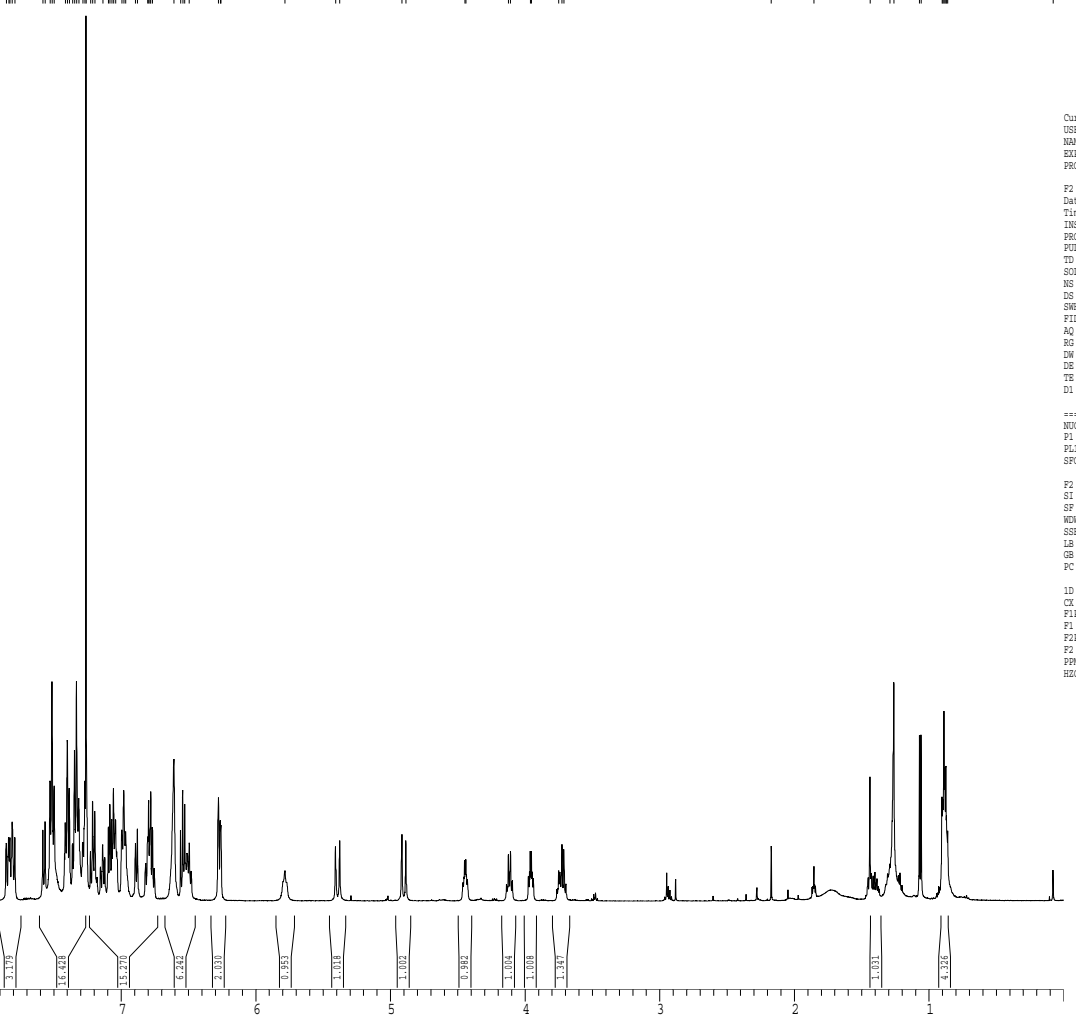

量
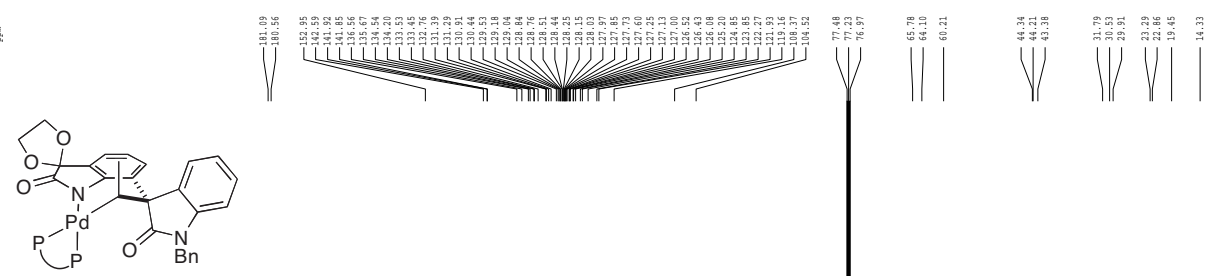

ent-3B: $[\mathrm{P}-\mathrm{P}]=(\mathrm{S})$-BINAP

${ }_{71} \mathrm{H}_{54} \mathrm{~N}_{2} \mathrm{O}_{4} \mathrm{P}_{2} \mathrm{Pd}$

Mol. Wt.: 1167.5670
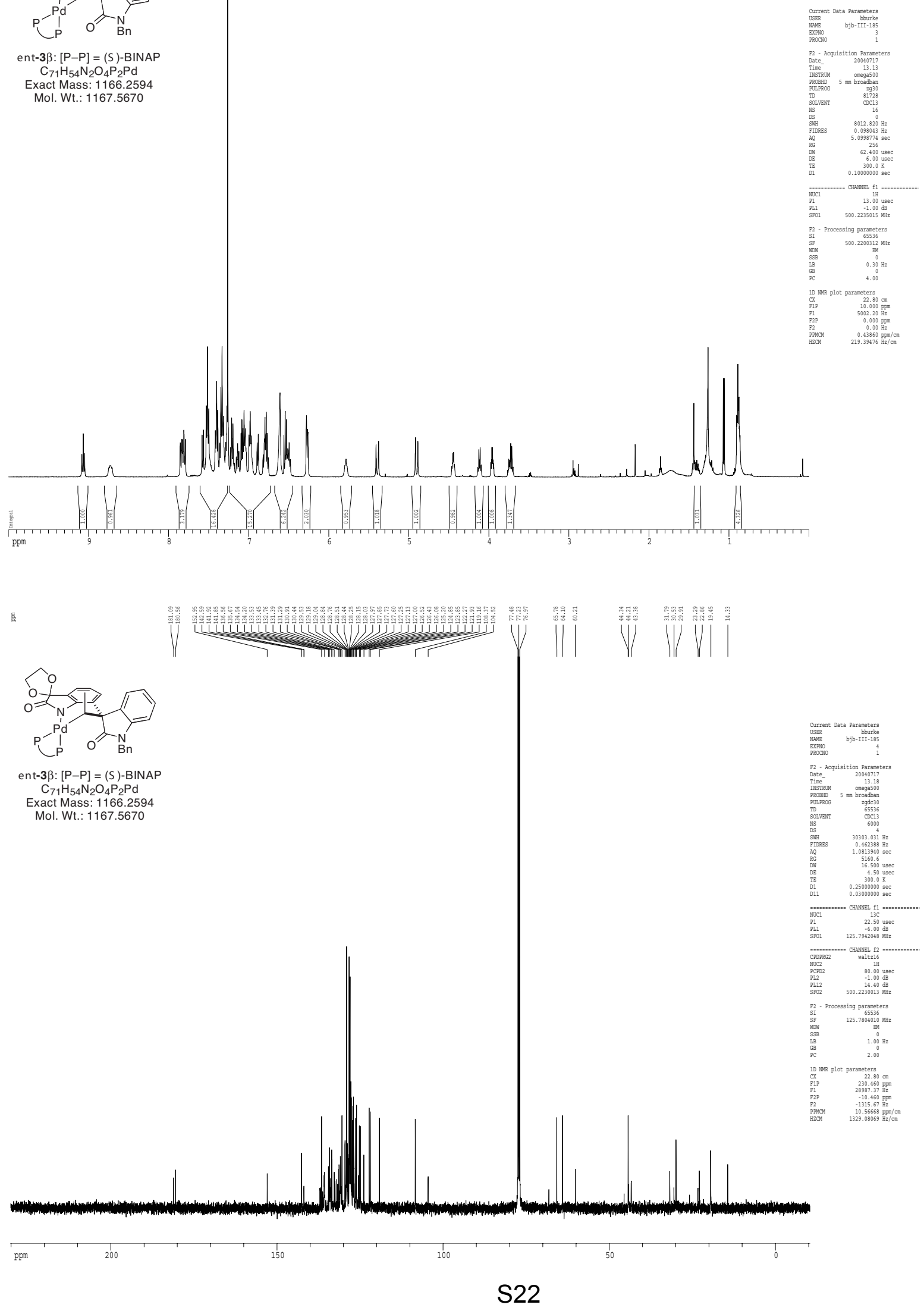


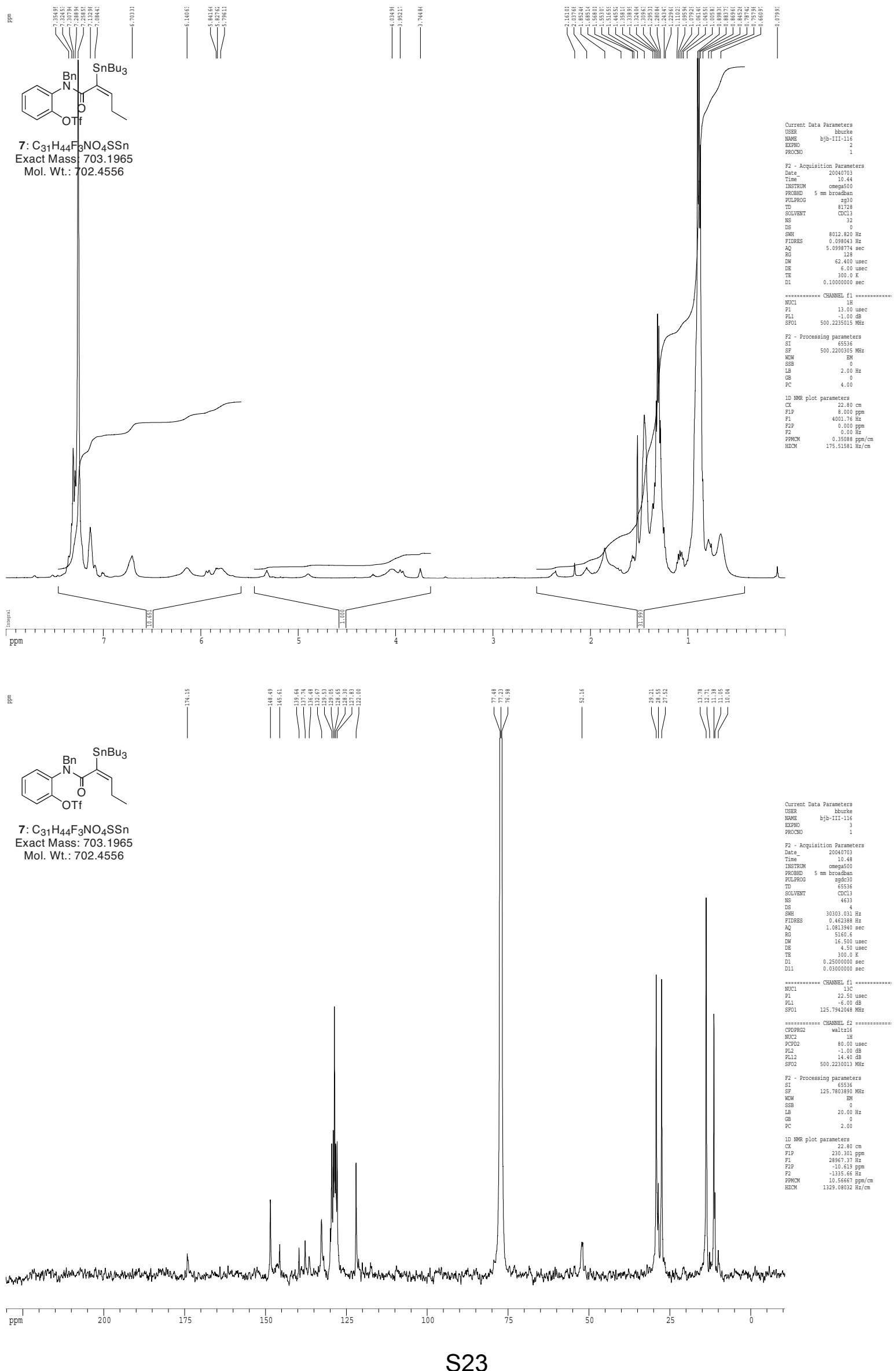




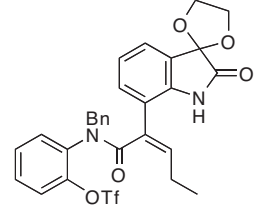

9: $\mathrm{C}_{29} \mathrm{H}_{25} \mathrm{~F}_{3} \mathrm{~N}_{2} \mathrm{O}_{7} \mathrm{~S}$ Exact Mass: 602.1335 Mol. Wt: 602.5782 10:1 mixture of amide rotamers
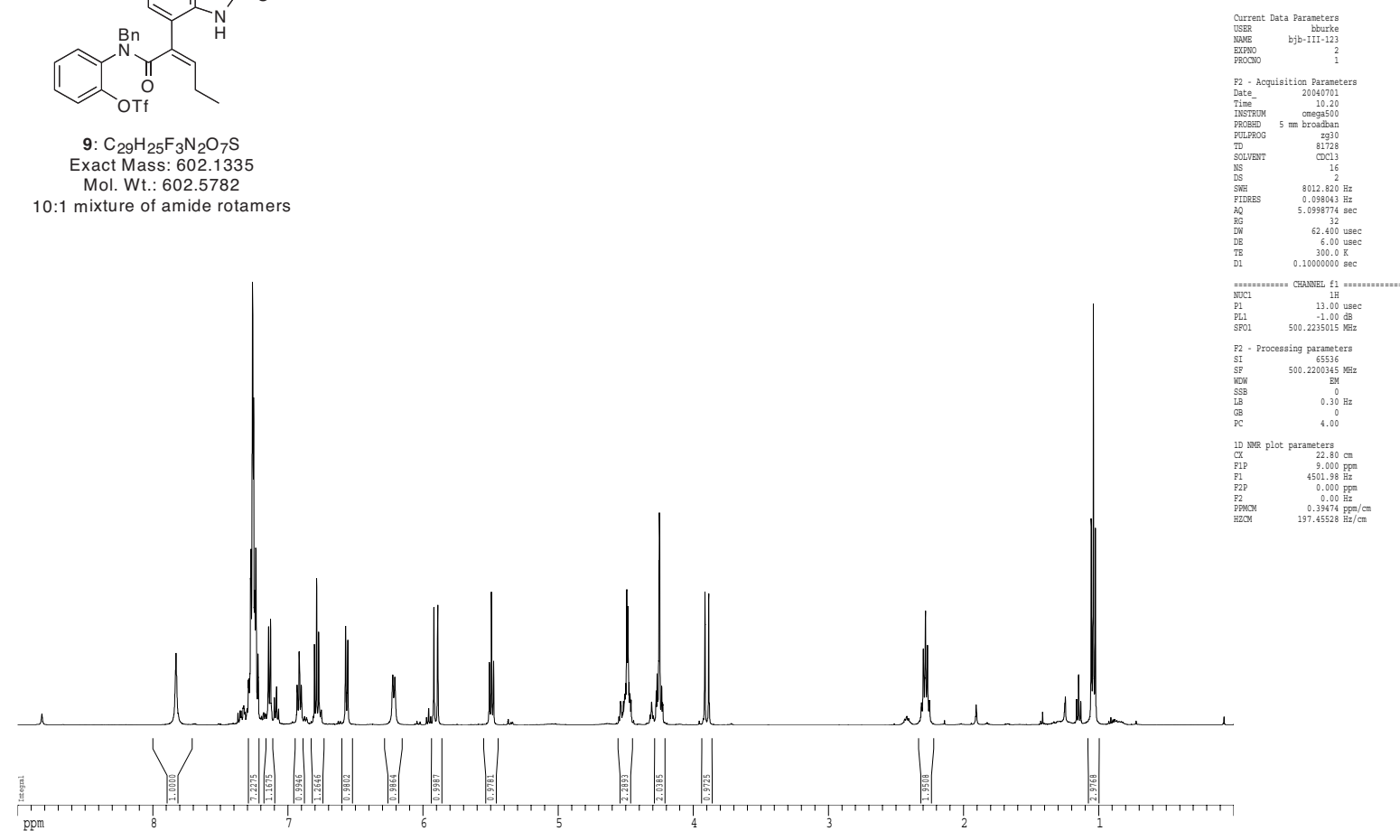

s:
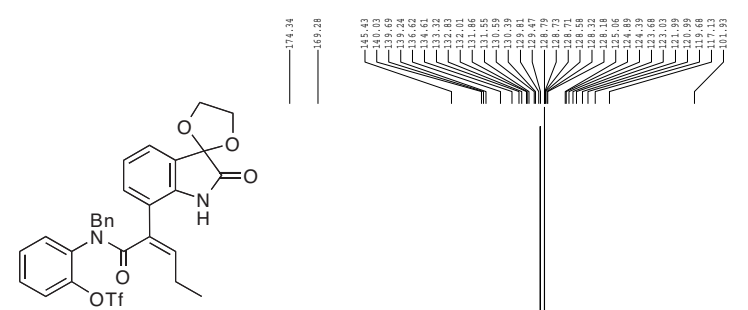

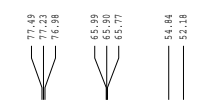

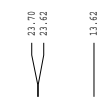

Mol. Wt.: 602.5782

10:1 mixture of amide rotamers
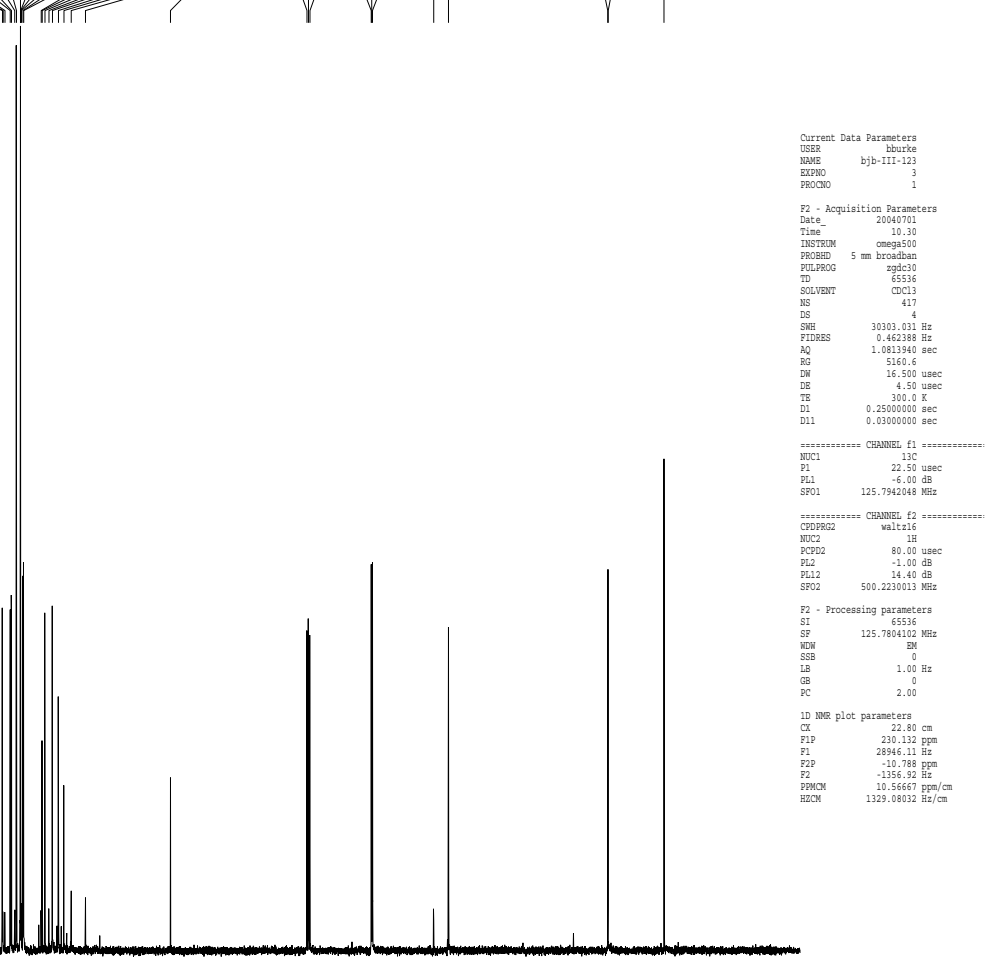

ppm

200

150

100

50 


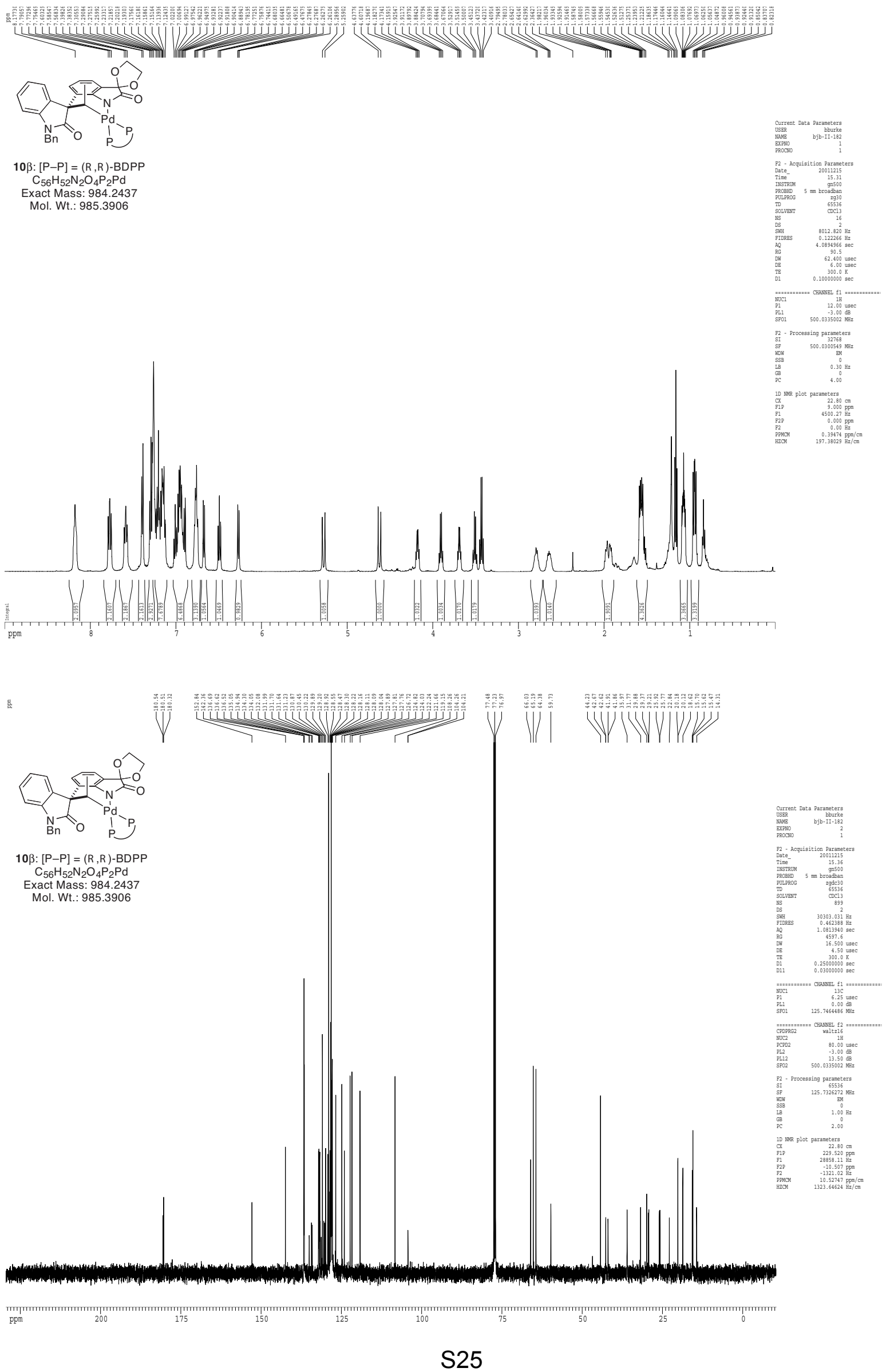




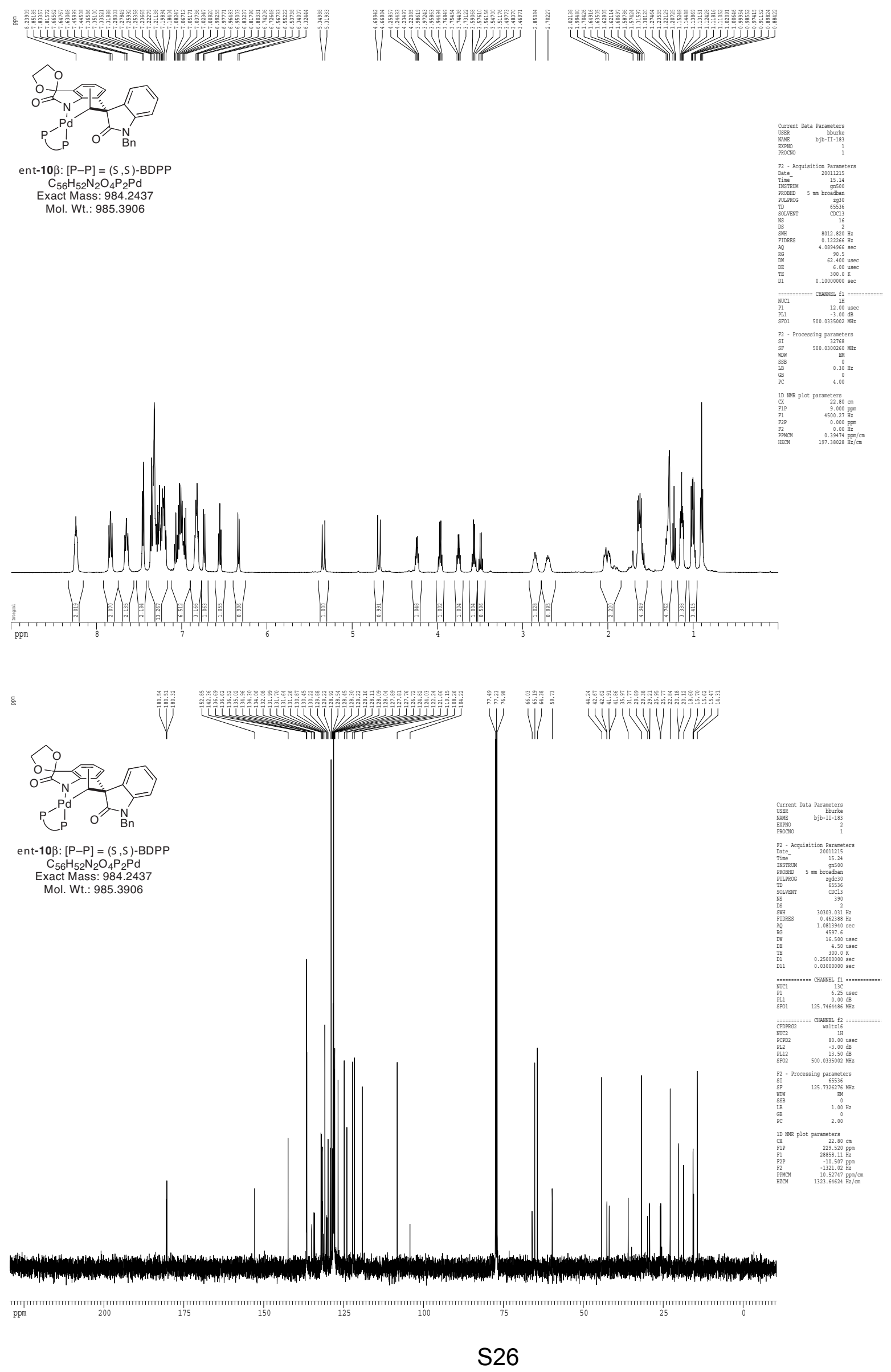




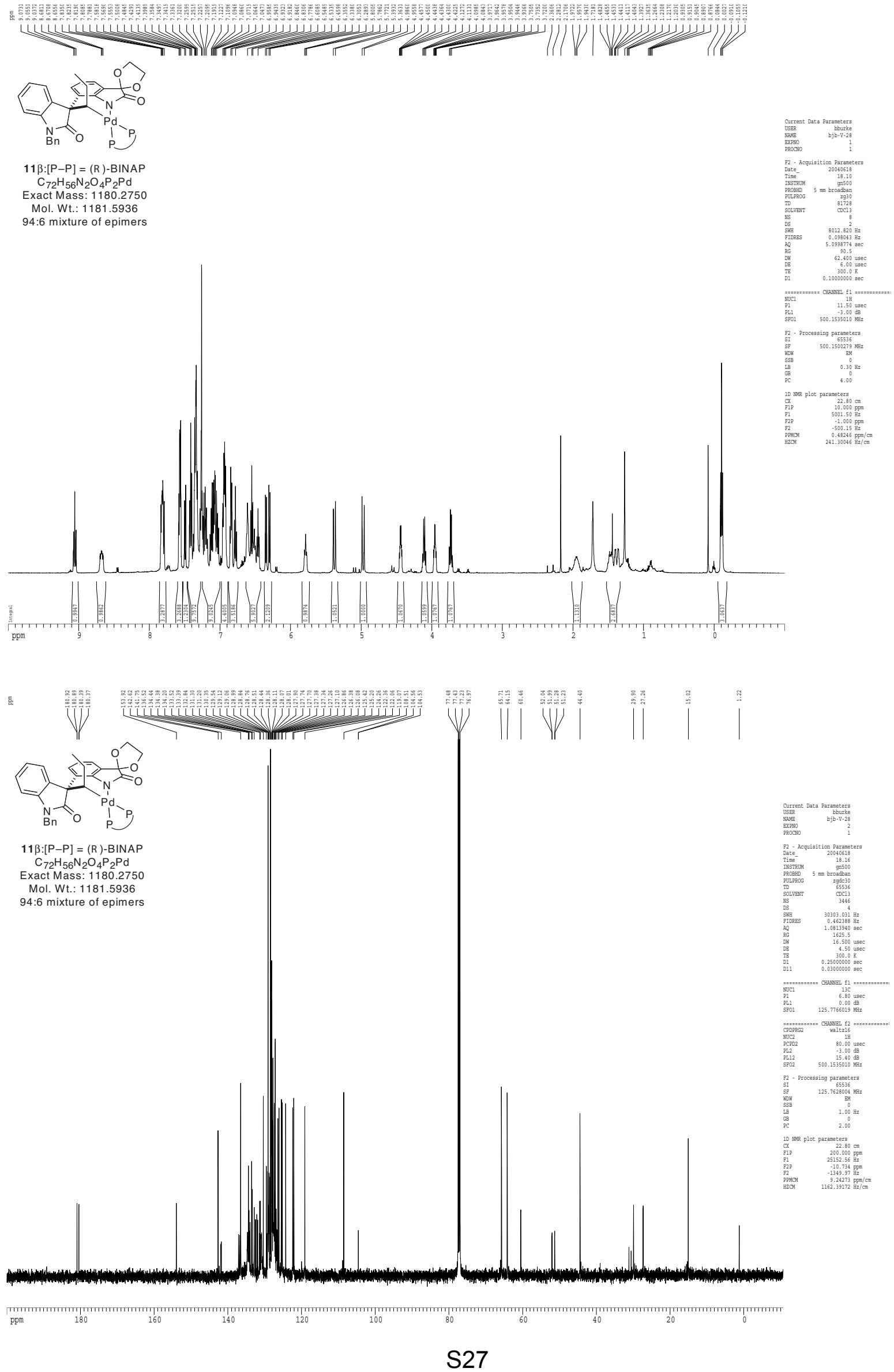


唇
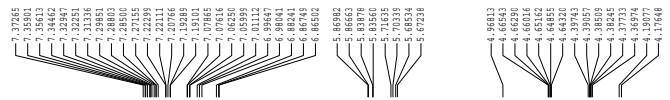

|
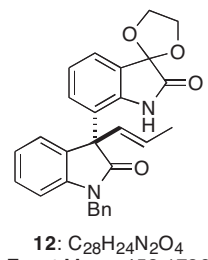

Exact Mass: 452.1736

Mol. Wt.: 452.5012
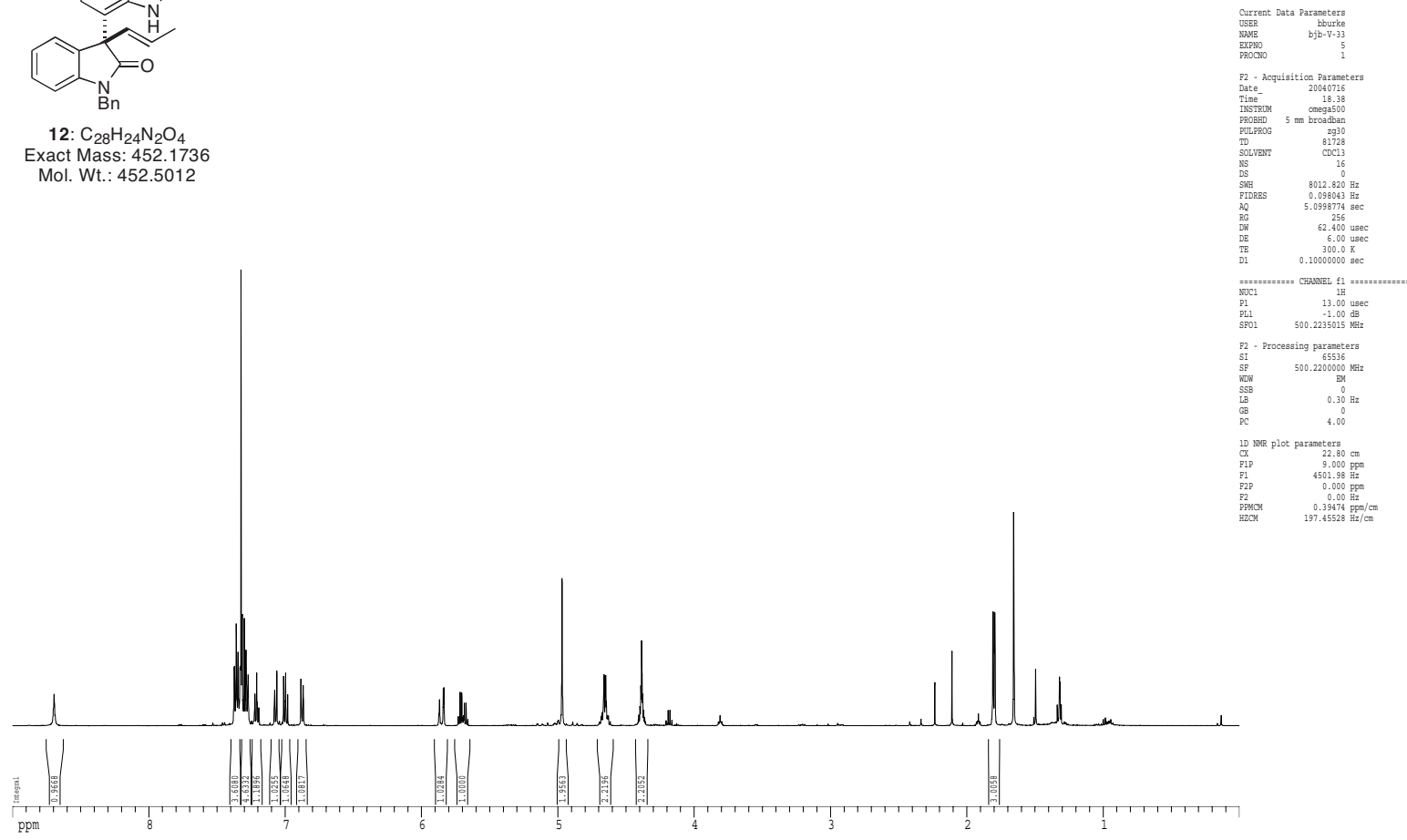

옹

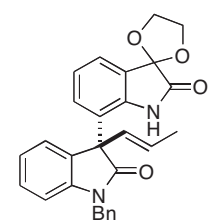

12: $\mathrm{C}_{28} \mathrm{H}_{24} \mathrm{~N}_{2} \mathrm{O}_{4}$ Exact Mass: 452.173

Mol. Wt.: 452.5012
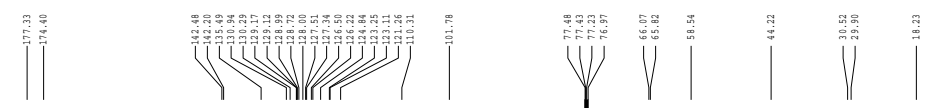

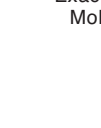

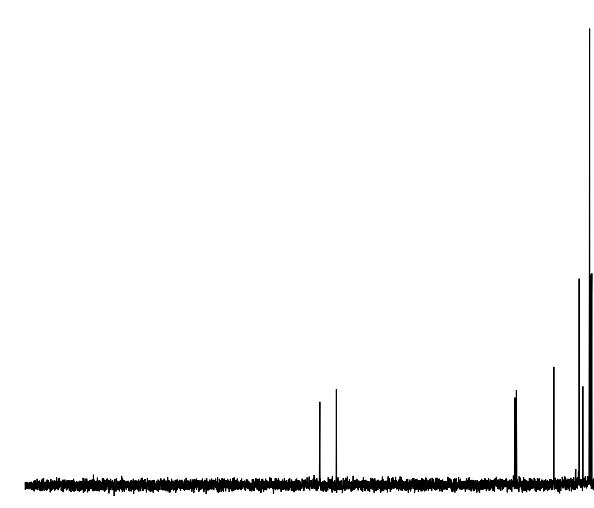

ppm

200

150

100

S28 

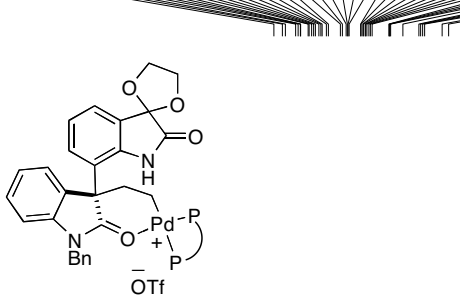

13: $[\mathrm{P}-\mathrm{P}]=(\mathrm{R})-\mathrm{BINAP}$

$\mathrm{C}_{71} \mathrm{H}_{55} \mathrm{~N}_{2} \mathrm{O}_{4} \mathrm{P}_{2} \mathrm{Pd}^{+}$

Exact Mass: 1167.2666

Mol. Wt.: 1168.5744
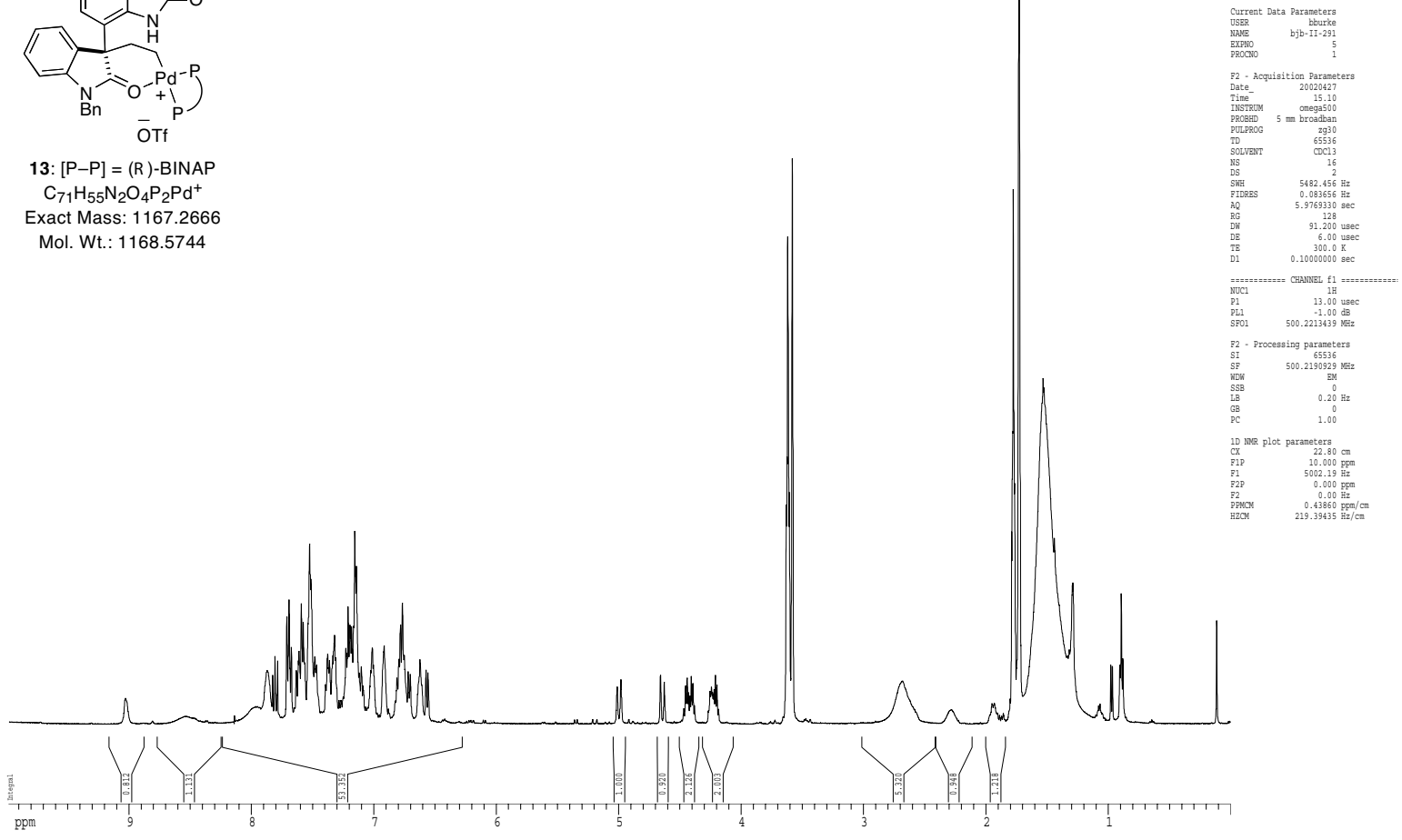

pp

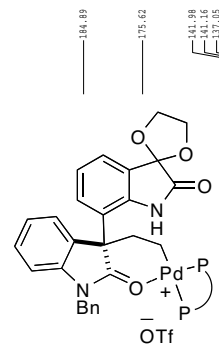

13: $[P-P]=(R)$-BINAP

$\mathrm{C}_{71} \mathrm{H}_{55} \mathrm{~N}_{2} \mathrm{O}_{4} \mathrm{P}_{2} \mathrm{Pd}^{+}$

Exact Mass: 1167.2666

Mol. Wt.: 1168.5744

contaminated with $\sim 20 \% 4$

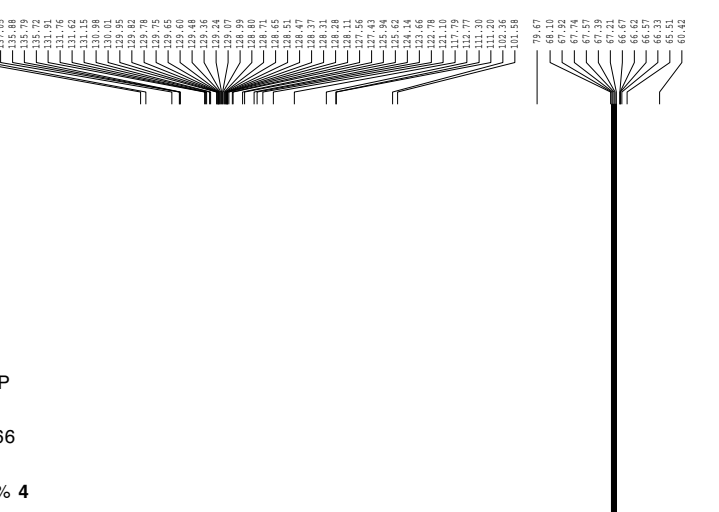

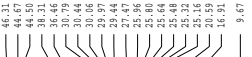

WVWW
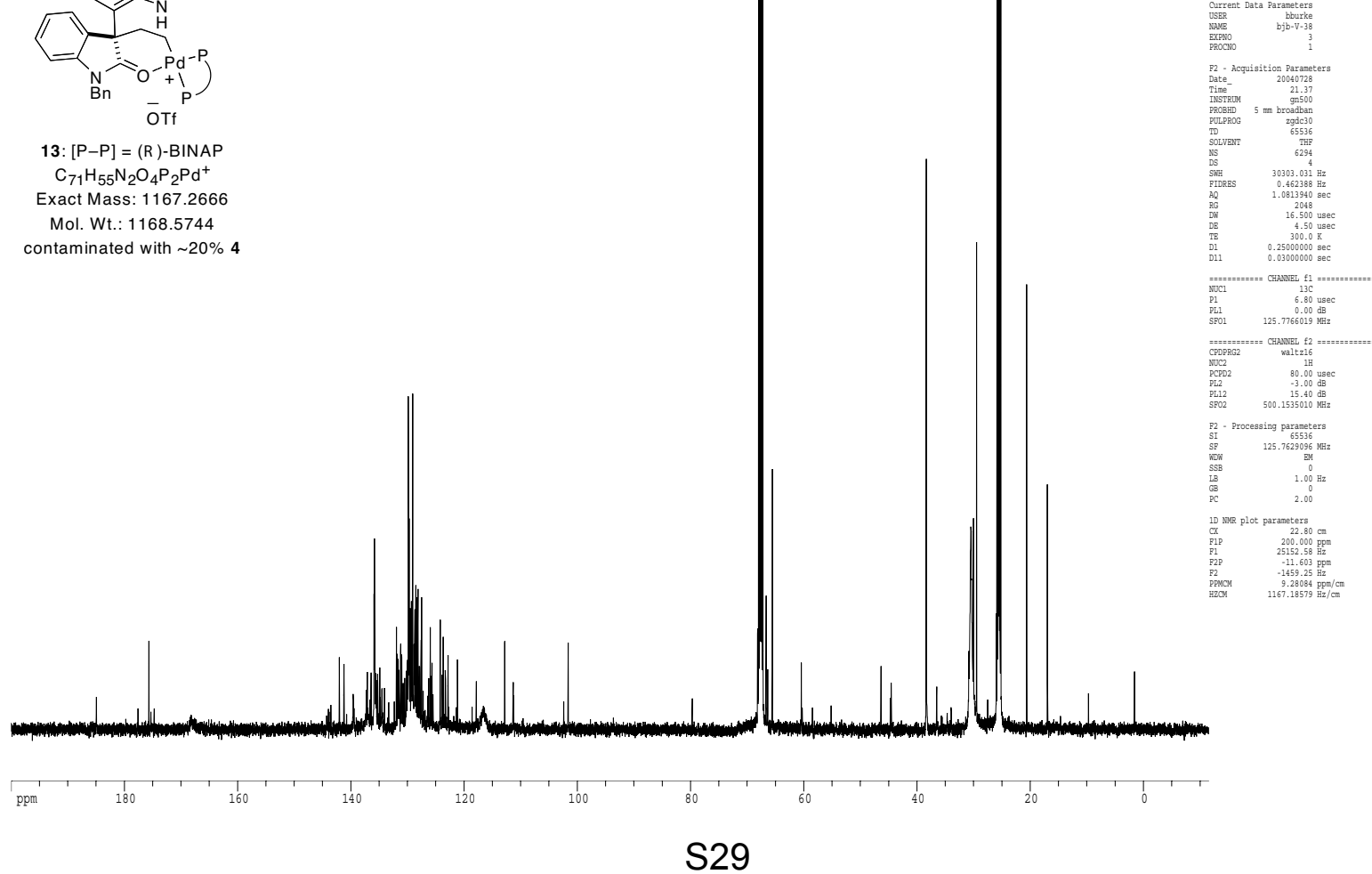
हิ

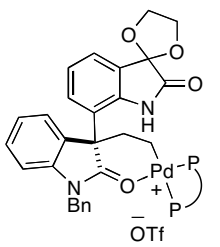

13: $[\mathrm{P}-\mathrm{P}]=(\mathrm{R})$-BINAP

$\mathrm{C}_{71} \mathrm{H}_{55} \mathrm{~N}_{2} \mathrm{O}_{4} \mathrm{P}_{2} \mathrm{Pd}^{+}$

Exact Mass: 1167.2666

Mol. Wt.: 1168.5744

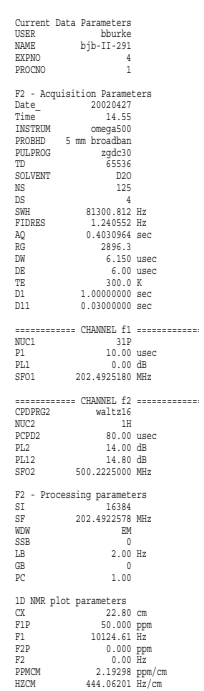

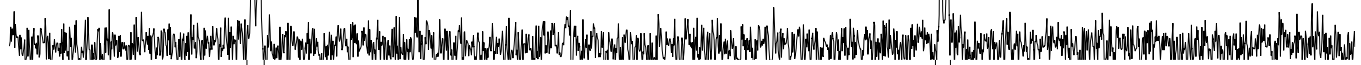
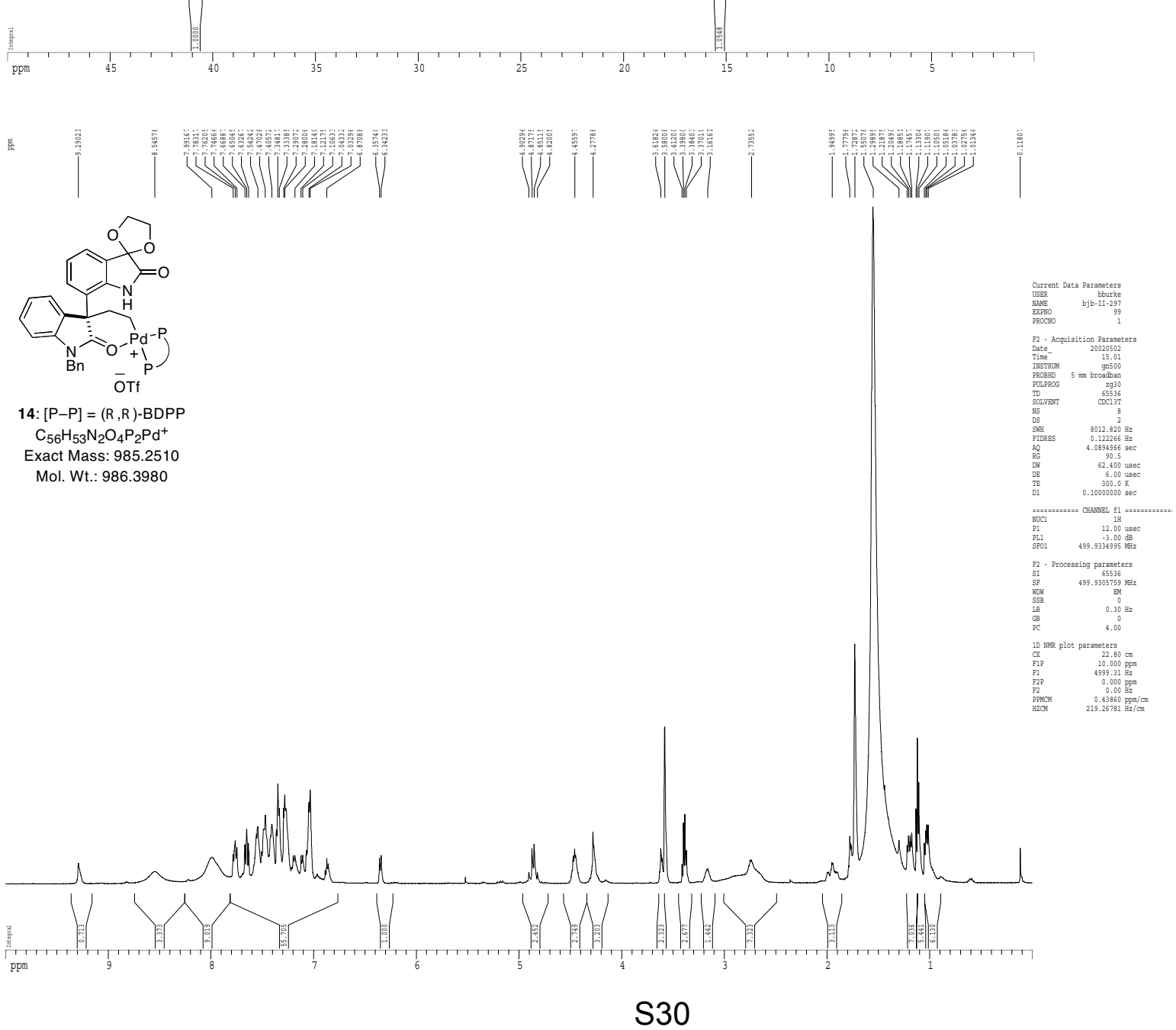


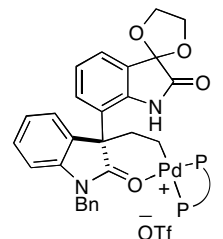

14: $[P-P]=(R, R)-B D P P$

$\mathrm{C}_{56} \mathrm{H}_{53} \mathrm{~N}_{2} \mathrm{O}_{4} \mathrm{P}_{2} \mathrm{Pd}^{+}$

Exact Mass: 985.2510

Mol. Wt.: 986.3980

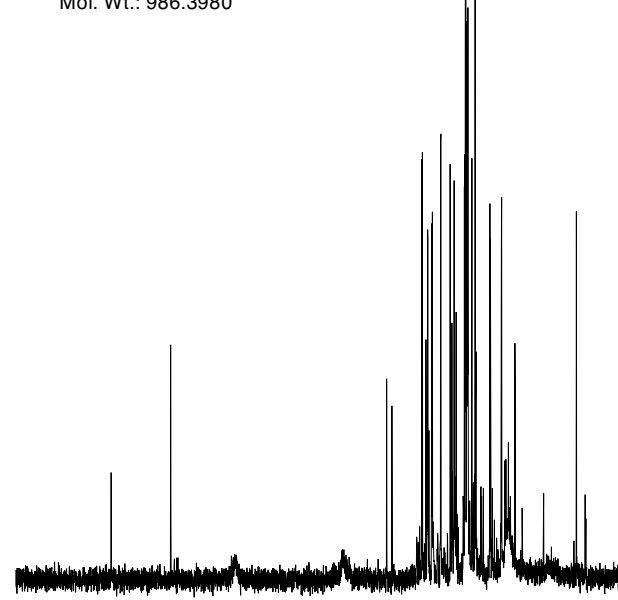

ppm

180

160140

120

100

80

60

40

骨
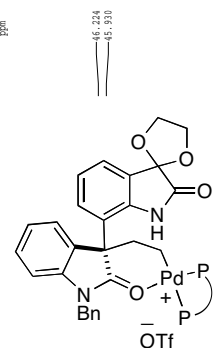

14: $[\mathrm{P}-\mathrm{P}]=(\mathrm{R}, \mathrm{R})-\mathrm{BDPP}$

$\mathrm{C}_{56} \mathrm{H}_{53} \mathrm{~N}_{2} \mathrm{O}_{4} \mathrm{P}_{2} \mathrm{Pd}^{+}$

Exact Mass: 985.2510

Mol. Wt.: 986.3980
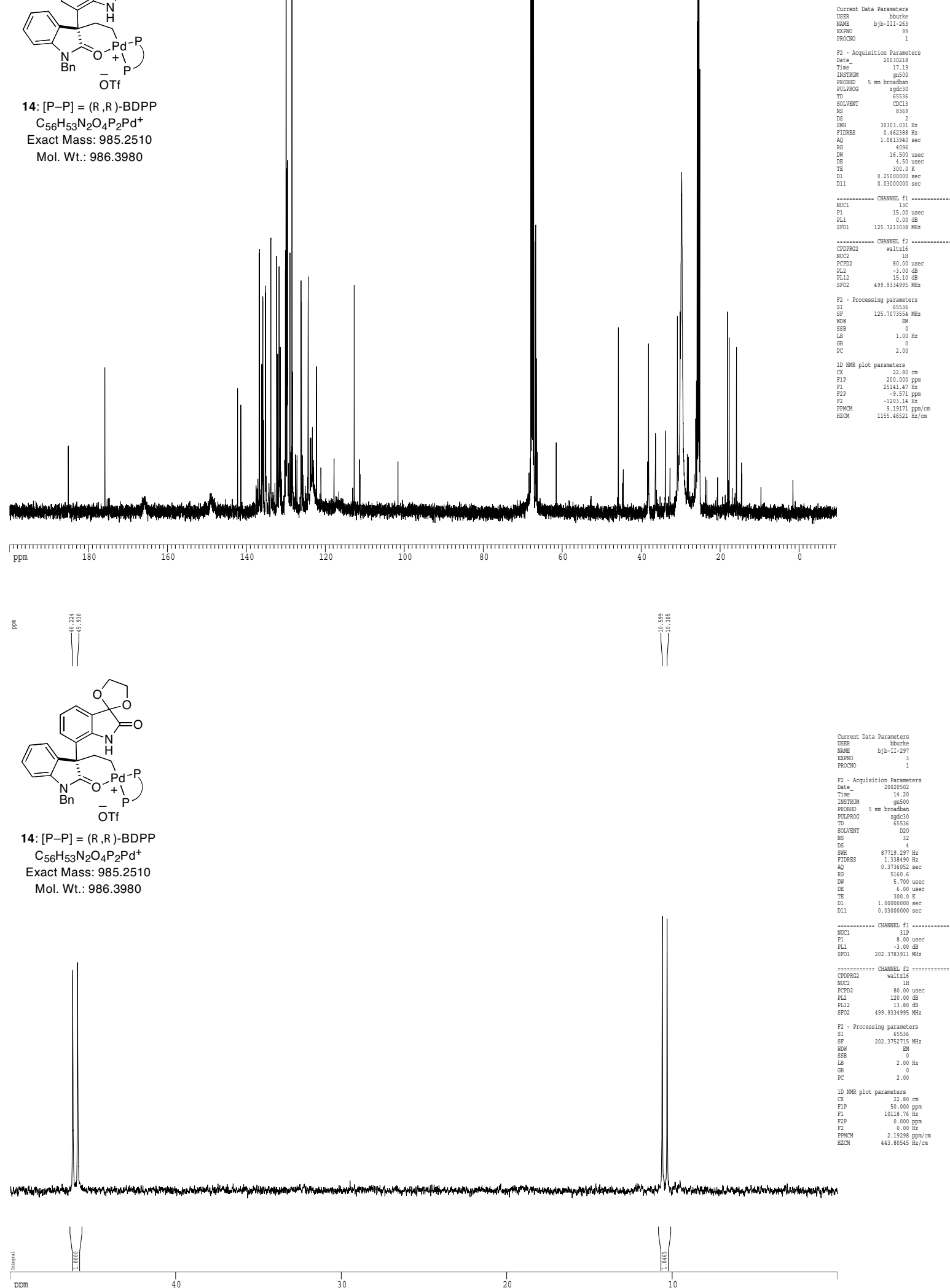


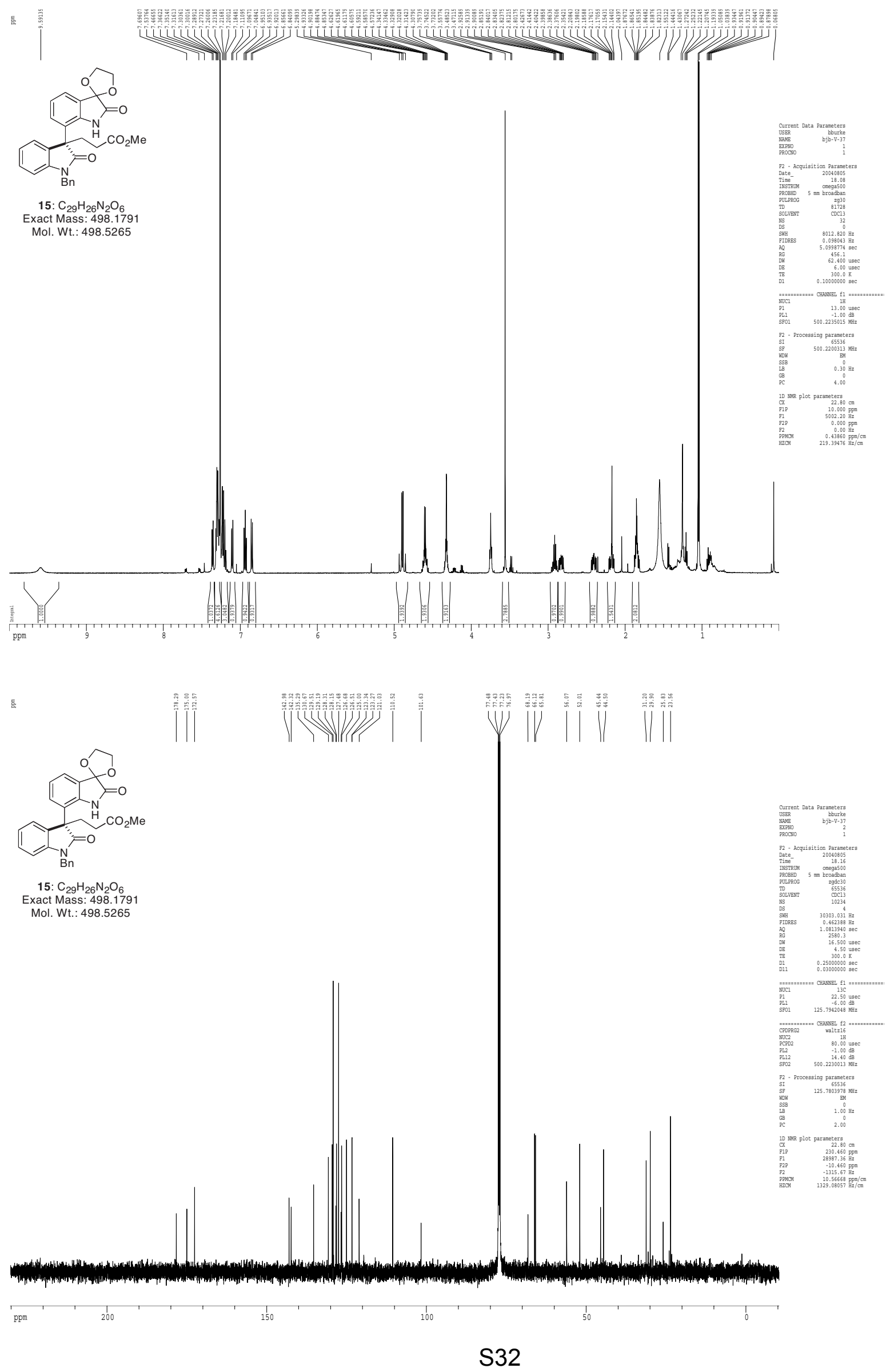




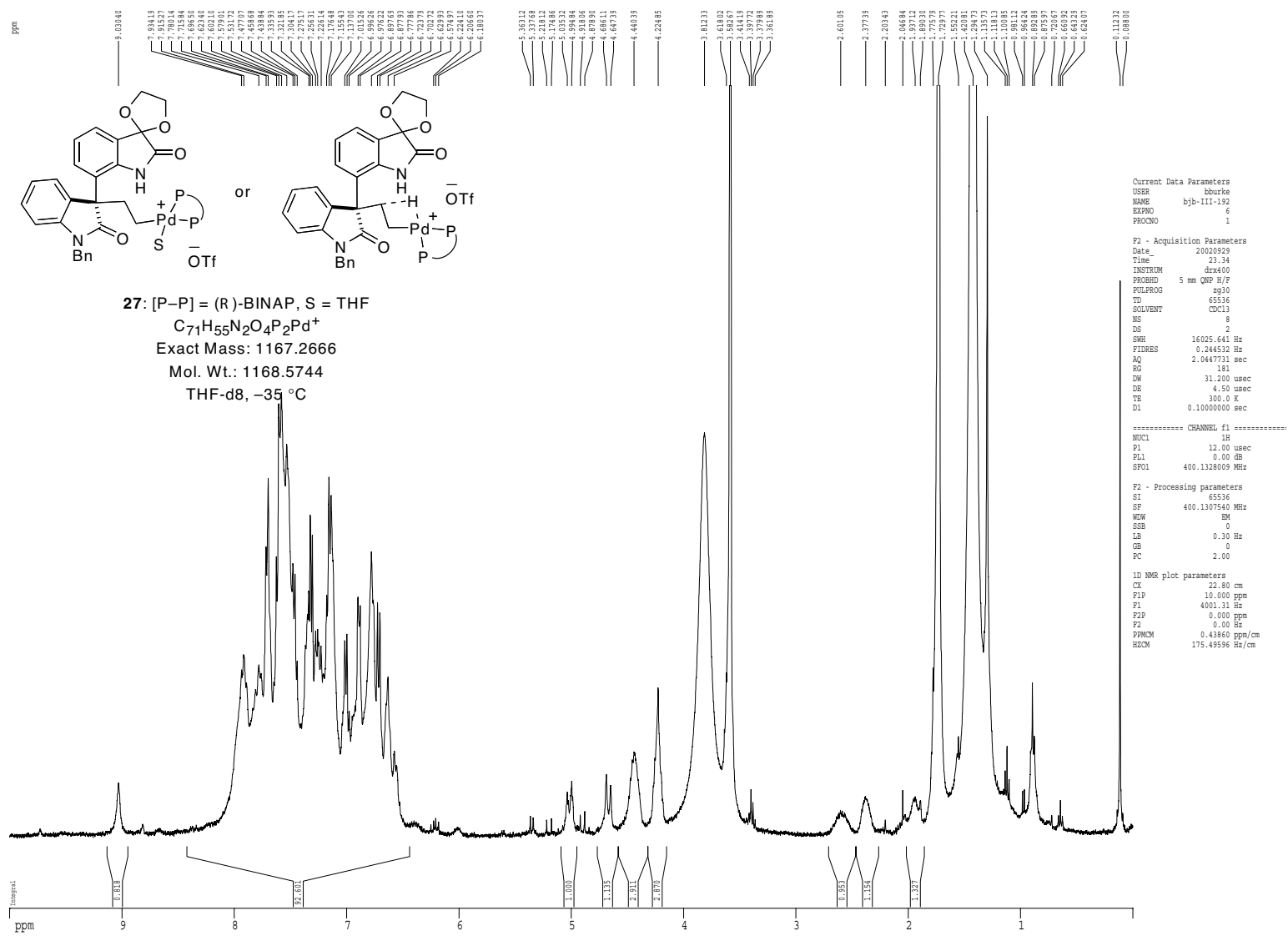

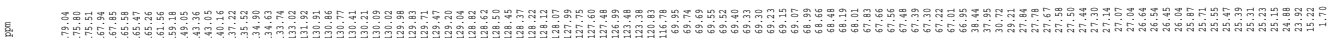

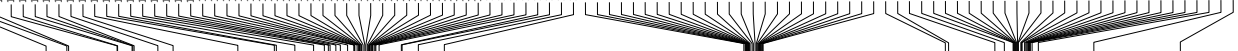

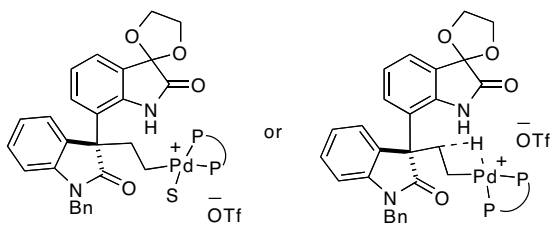

27: $[\mathrm{P}-\mathrm{P}]=(\mathrm{R})$-BINAP, $\mathrm{S}=\mathrm{THF}$ $\mathrm{C}_{71} \mathrm{H}_{55} \mathrm{~N}_{2} \mathrm{O}_{4} \mathrm{P}_{2} \mathrm{Pd}^{+}$

Exact Mass: 1167.2666 Mol. Wt.: 1168.5744

THF-d8, $-35^{\circ} \mathrm{C}$
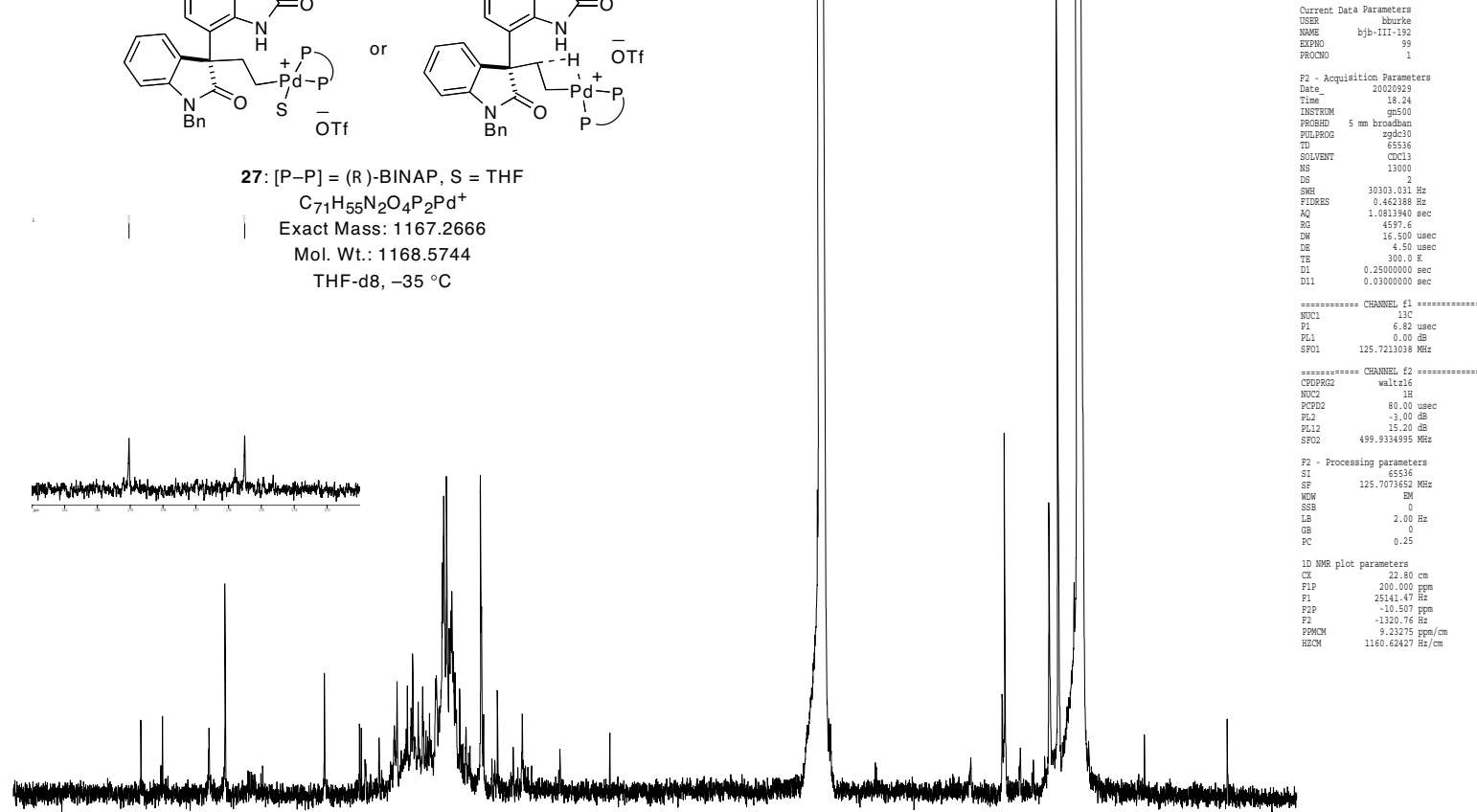

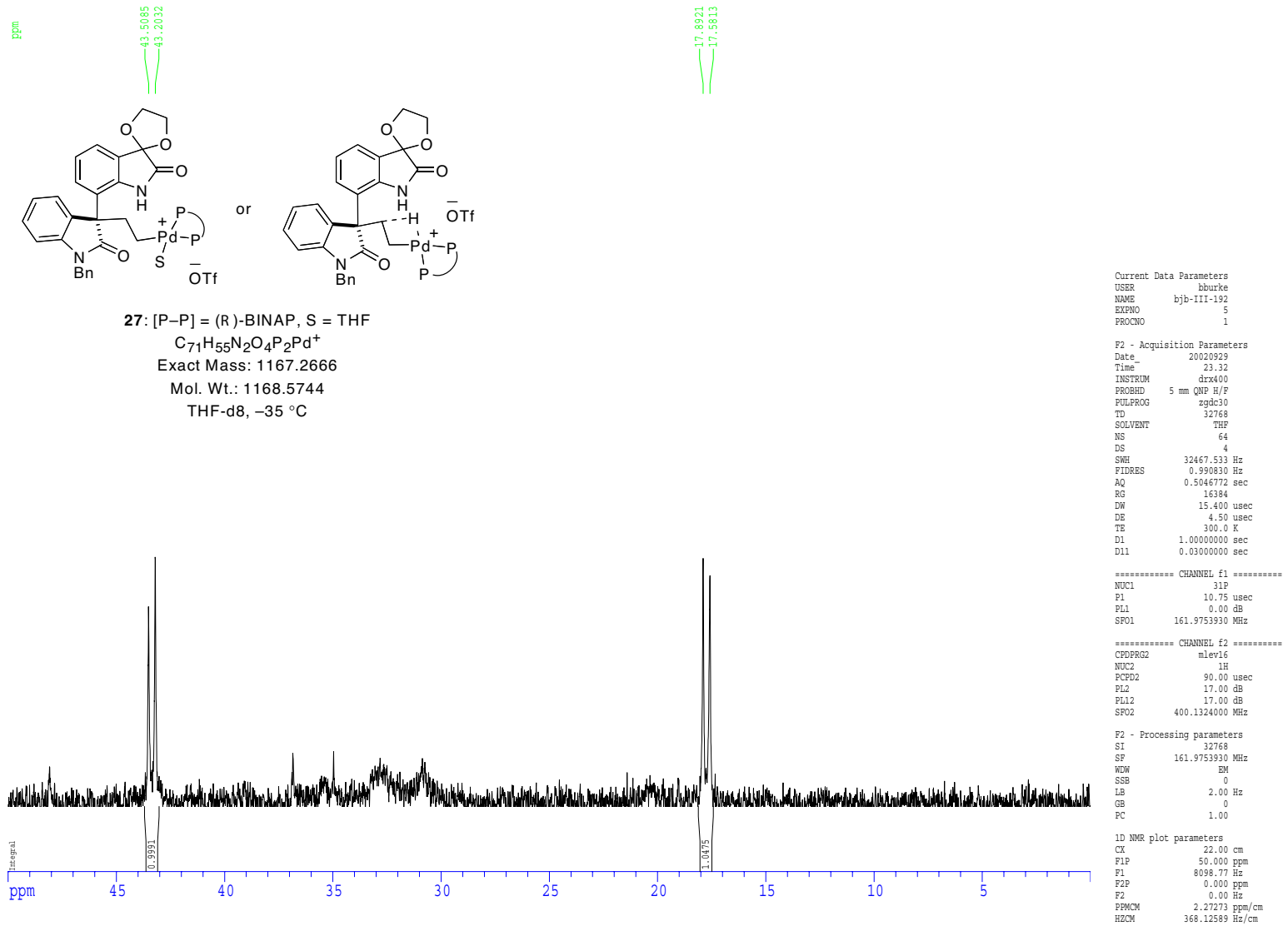


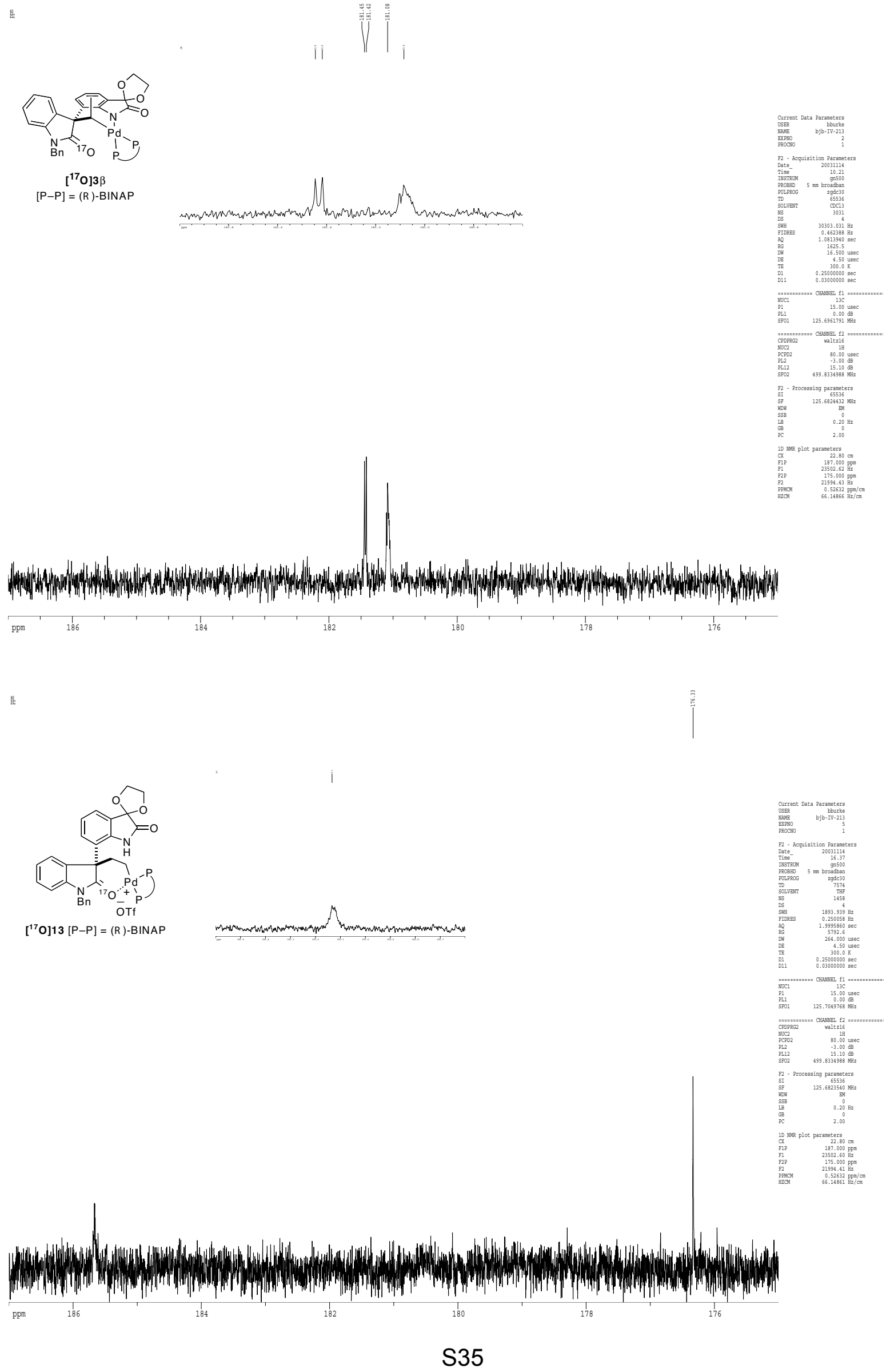



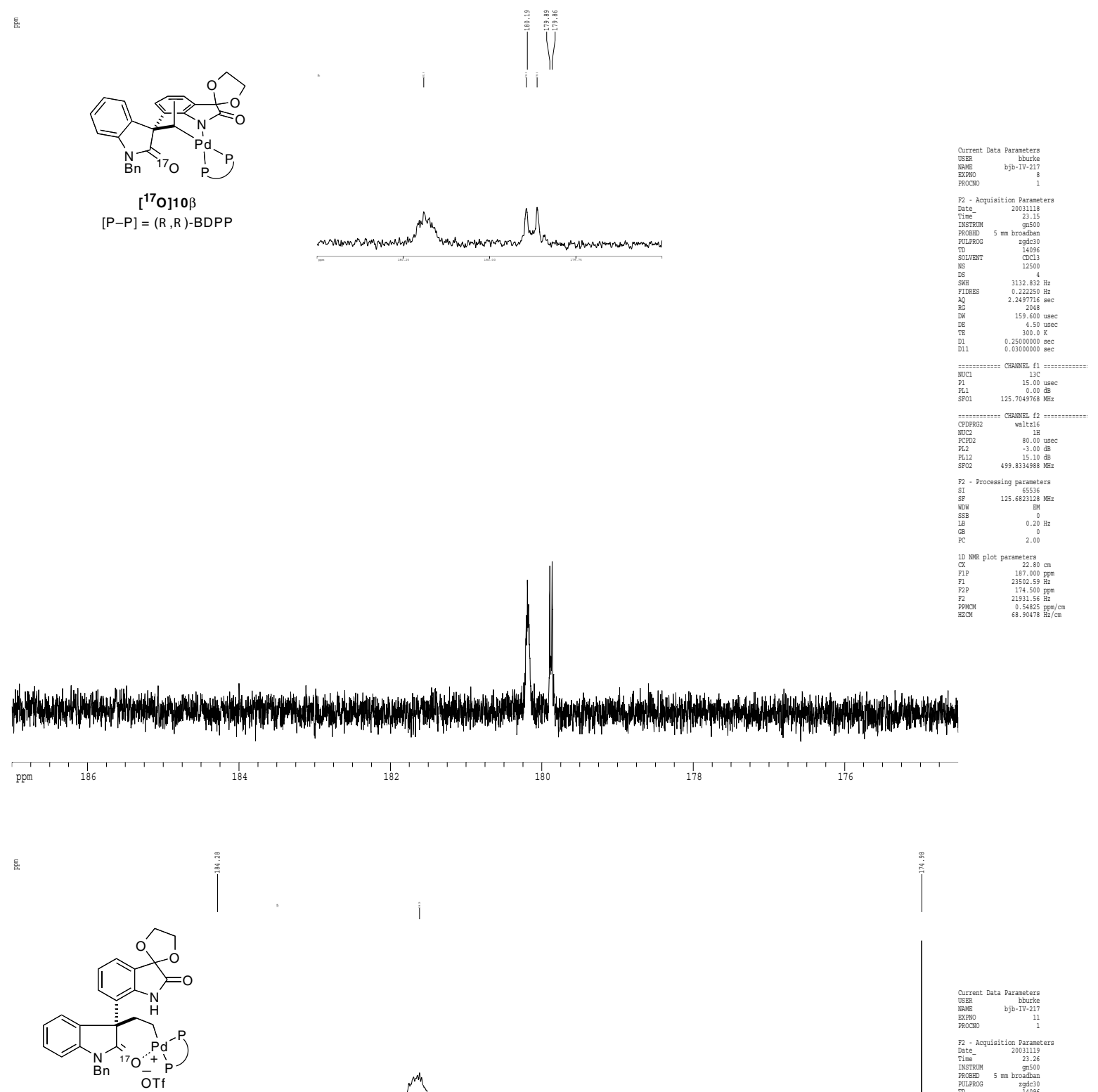

$\left[{ }^{17} \mathrm{O}\right] 14[\mathrm{P}-\mathrm{P}]=(\mathrm{R}, \mathrm{R})-\mathrm{BDPP}$
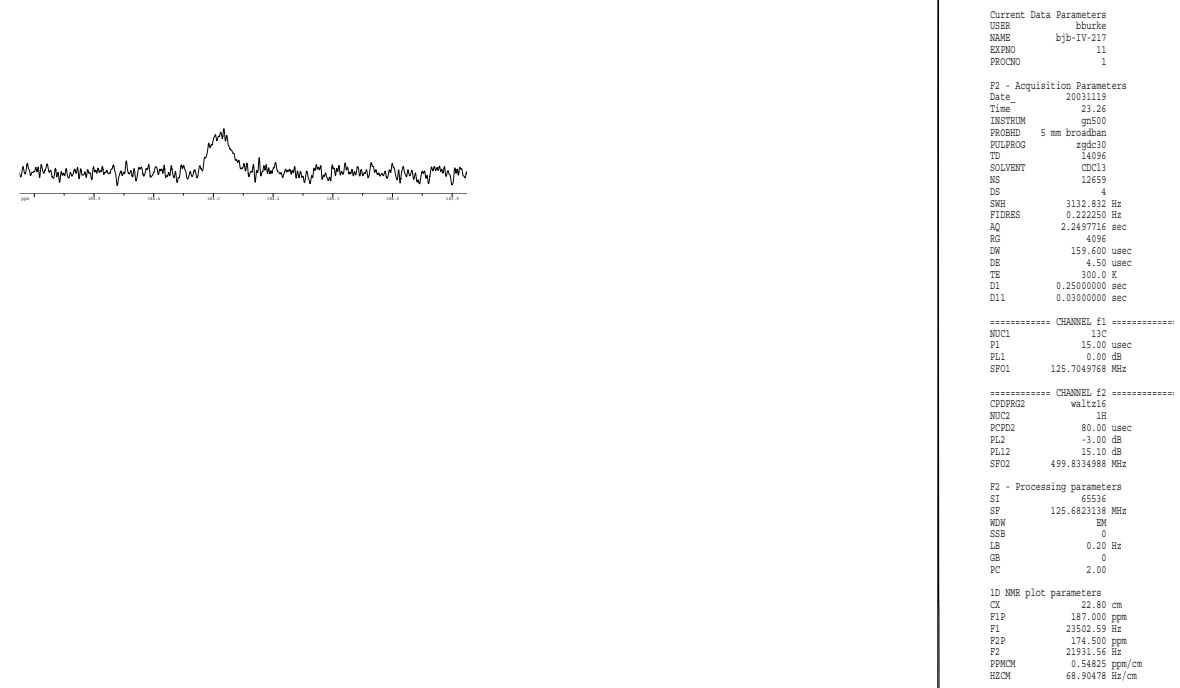

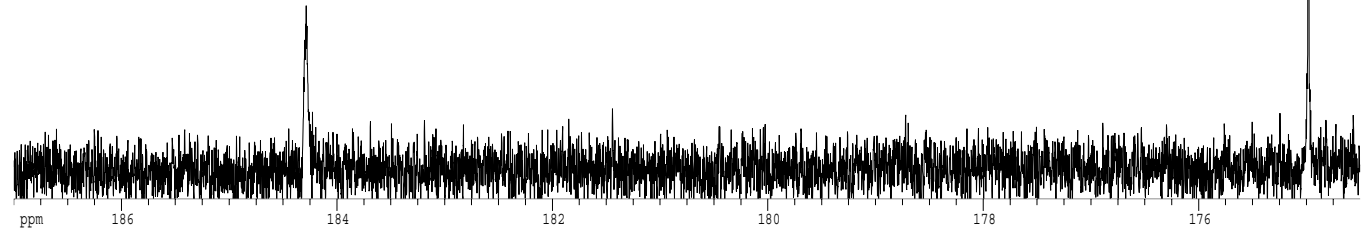


BINAP palladacycle high res

bjb-V -42

$08 / 6 / 2004$

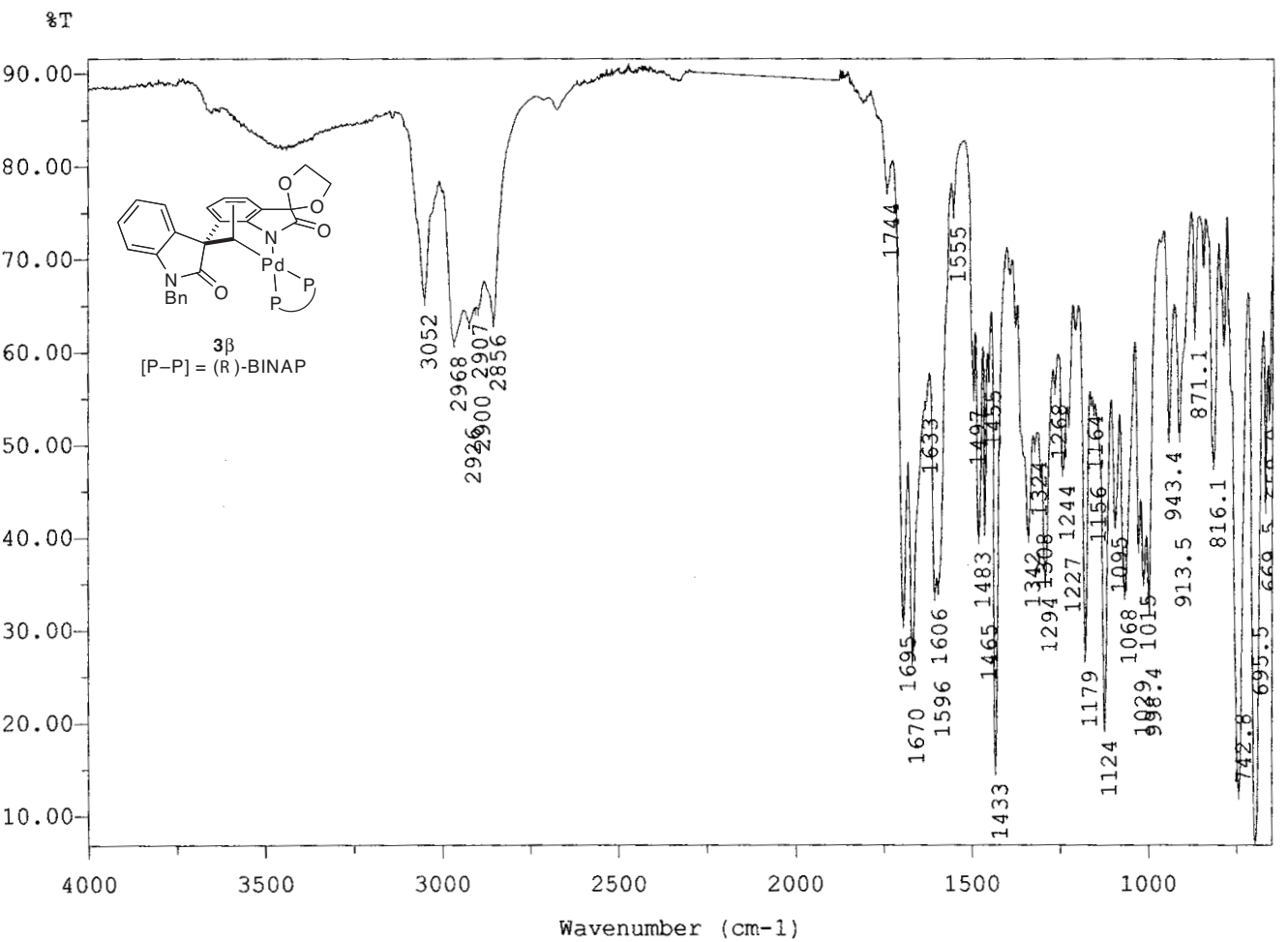

cationic BINAP pallad. high res II

bjb-V -44

$08 / 7 / 2004$

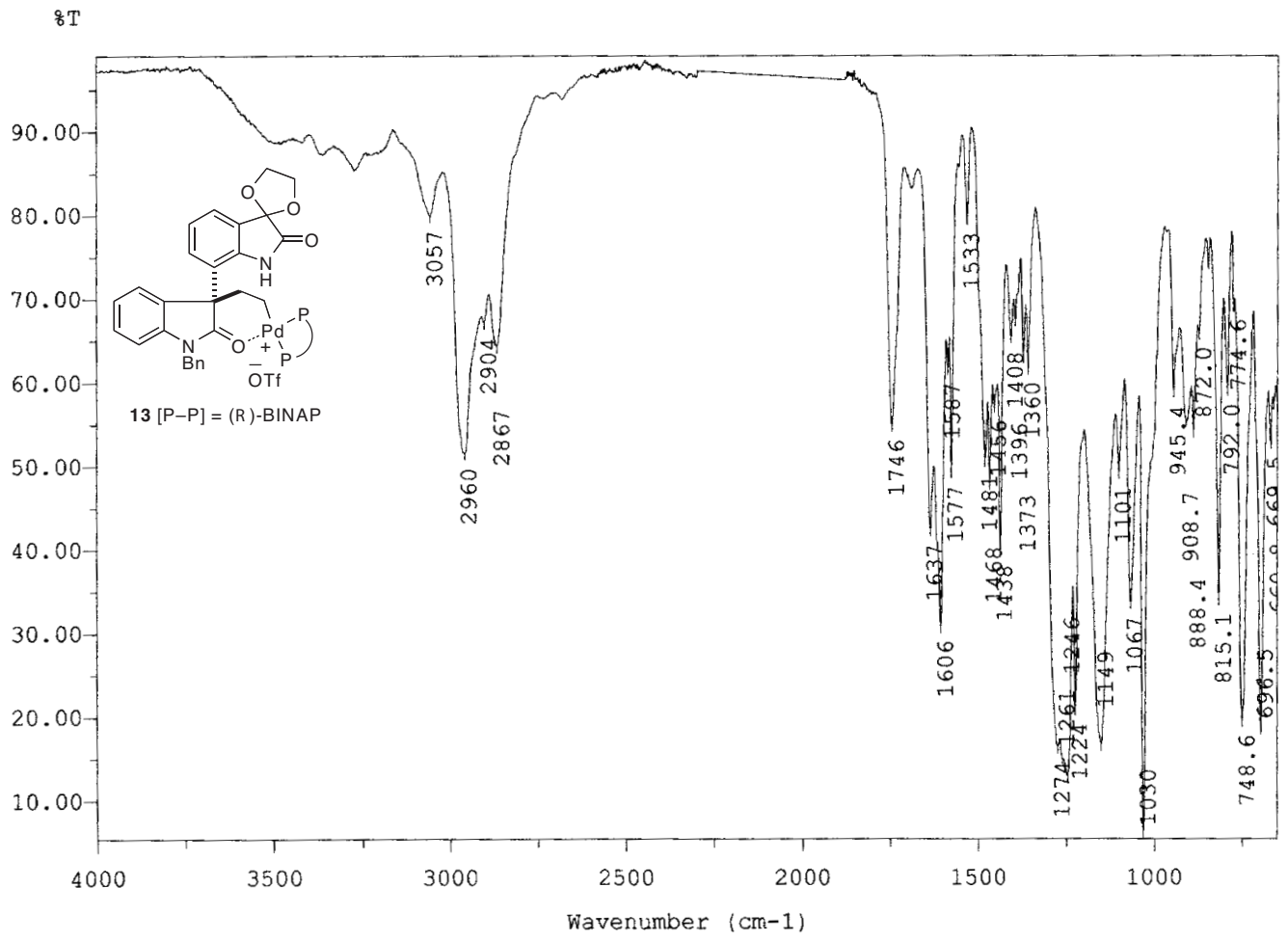


170 BINAP palladacycle high res

$b j b-v-42$

$08 / 6 / 2004$

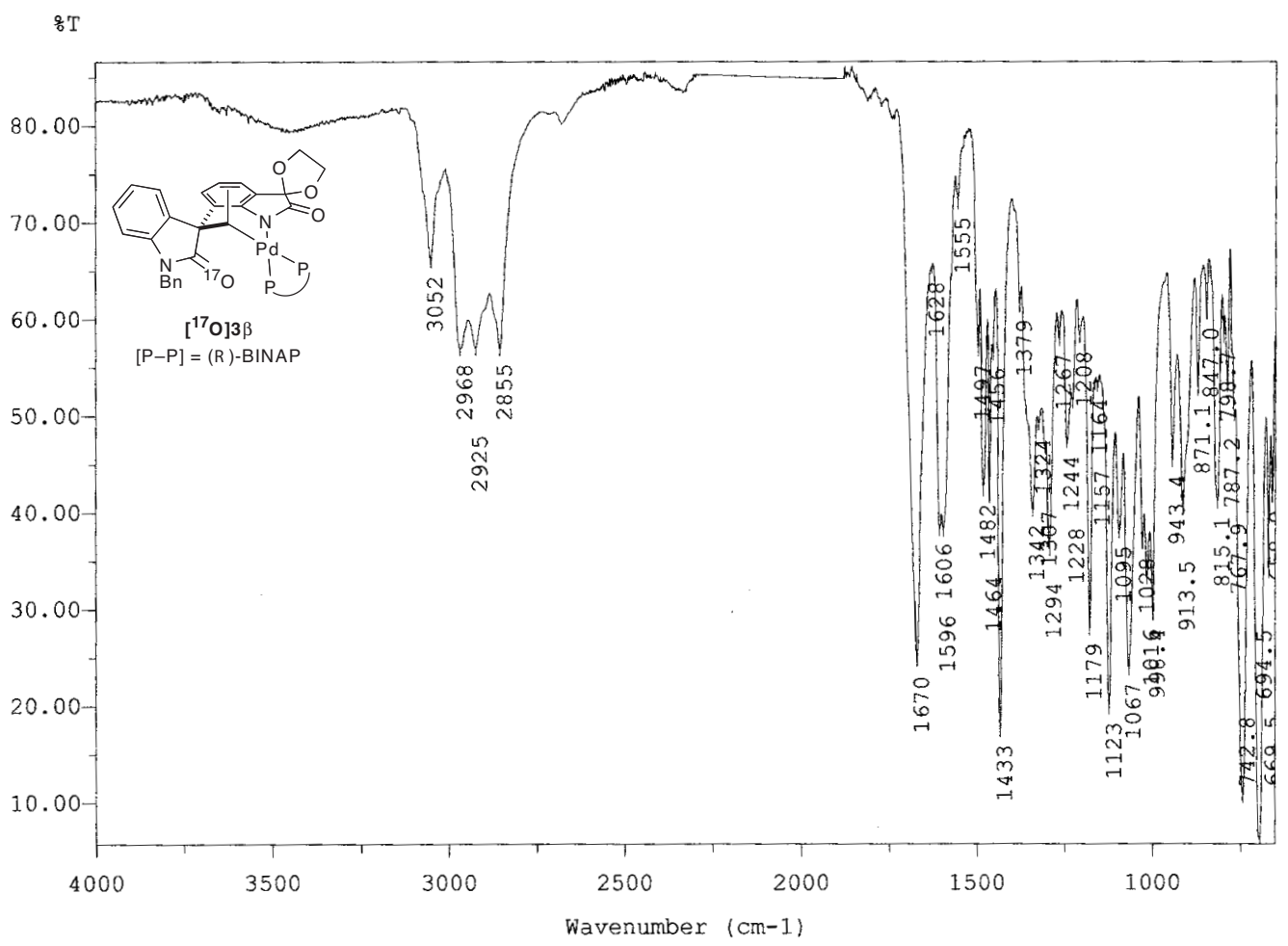

cationic 170 BINAP palladacycle high res

bjb $-V-42$

$08 / 6 / 2004$

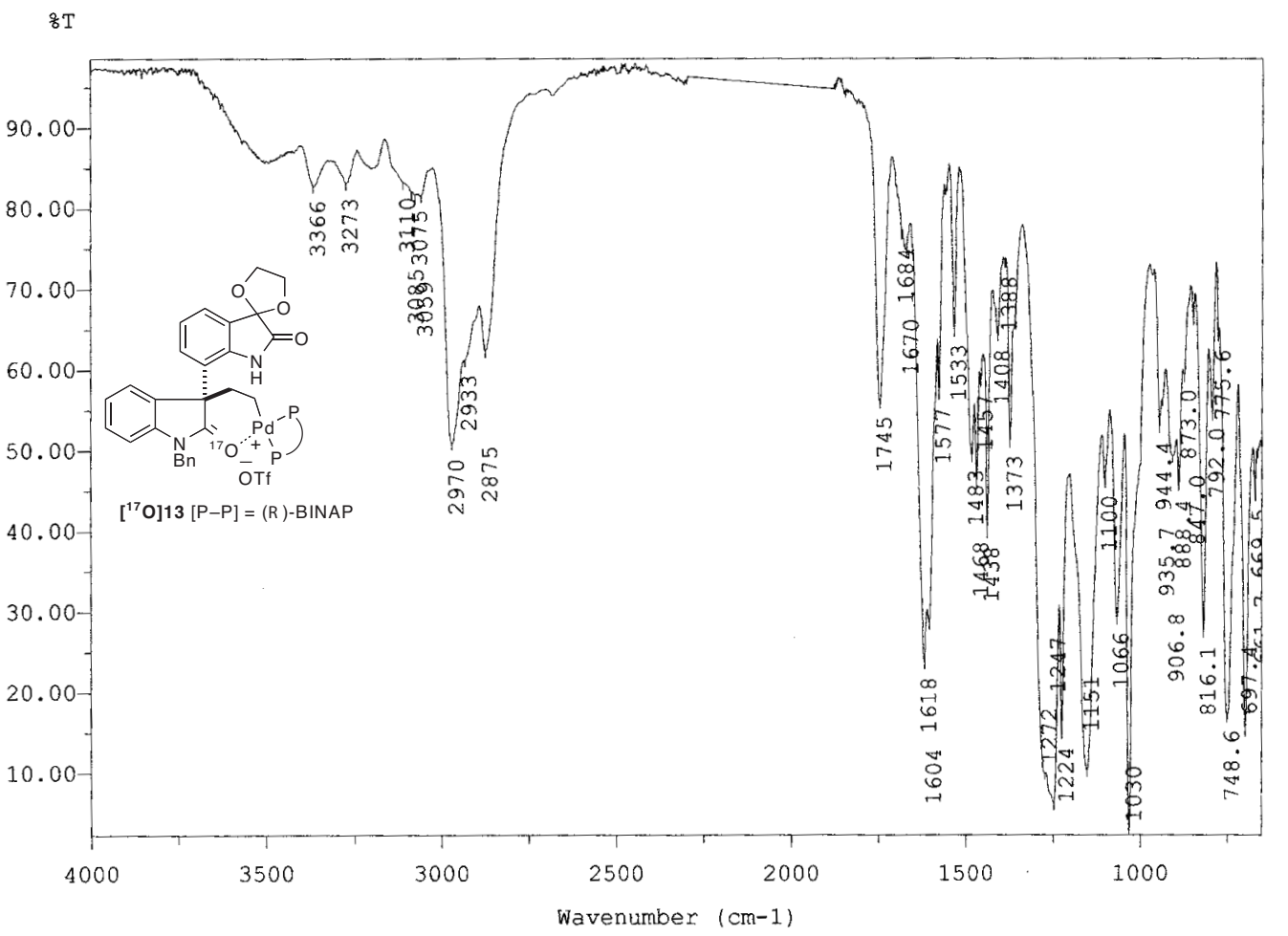


BDPP palladacycle high res

bjb-v -41

$08 / 6 / 2004$

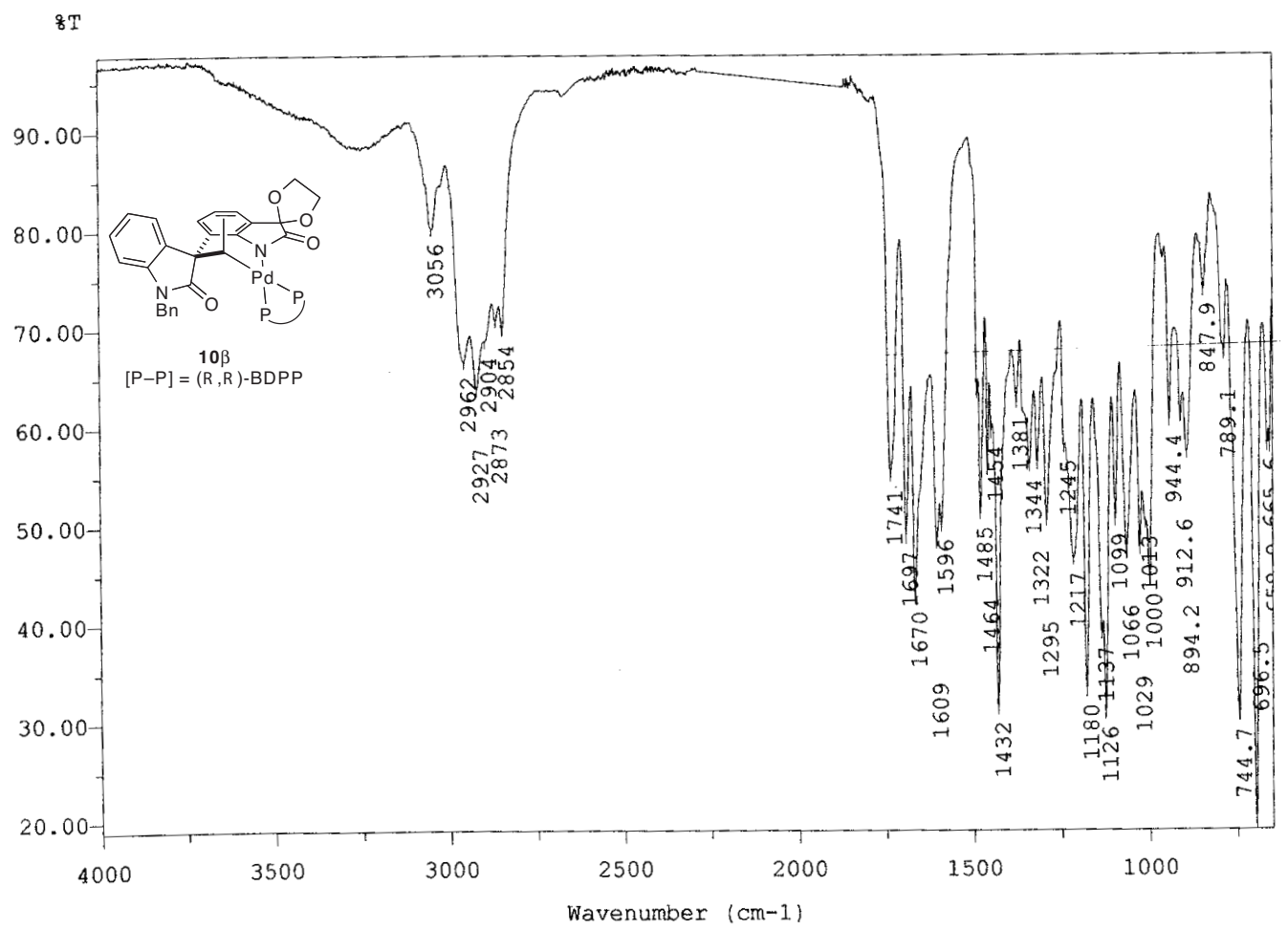

cationic BDPP palladacycle high res

bjb-V-41

$08 / 6 / 2004$

웅 $\mathrm{T}$

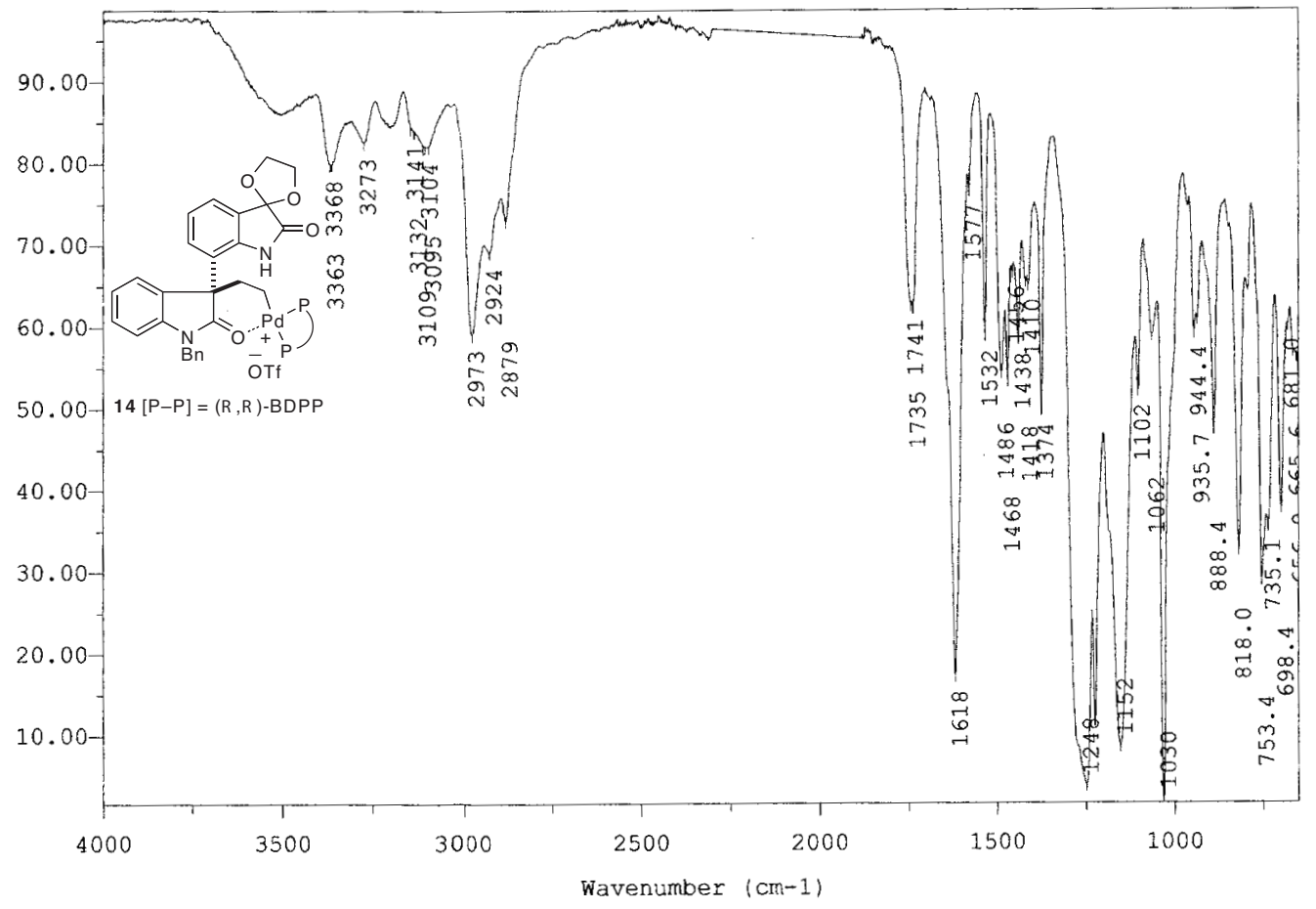


170 BDPP palladacycle high res

bjb-V-41

$08 / 6 / 2004$

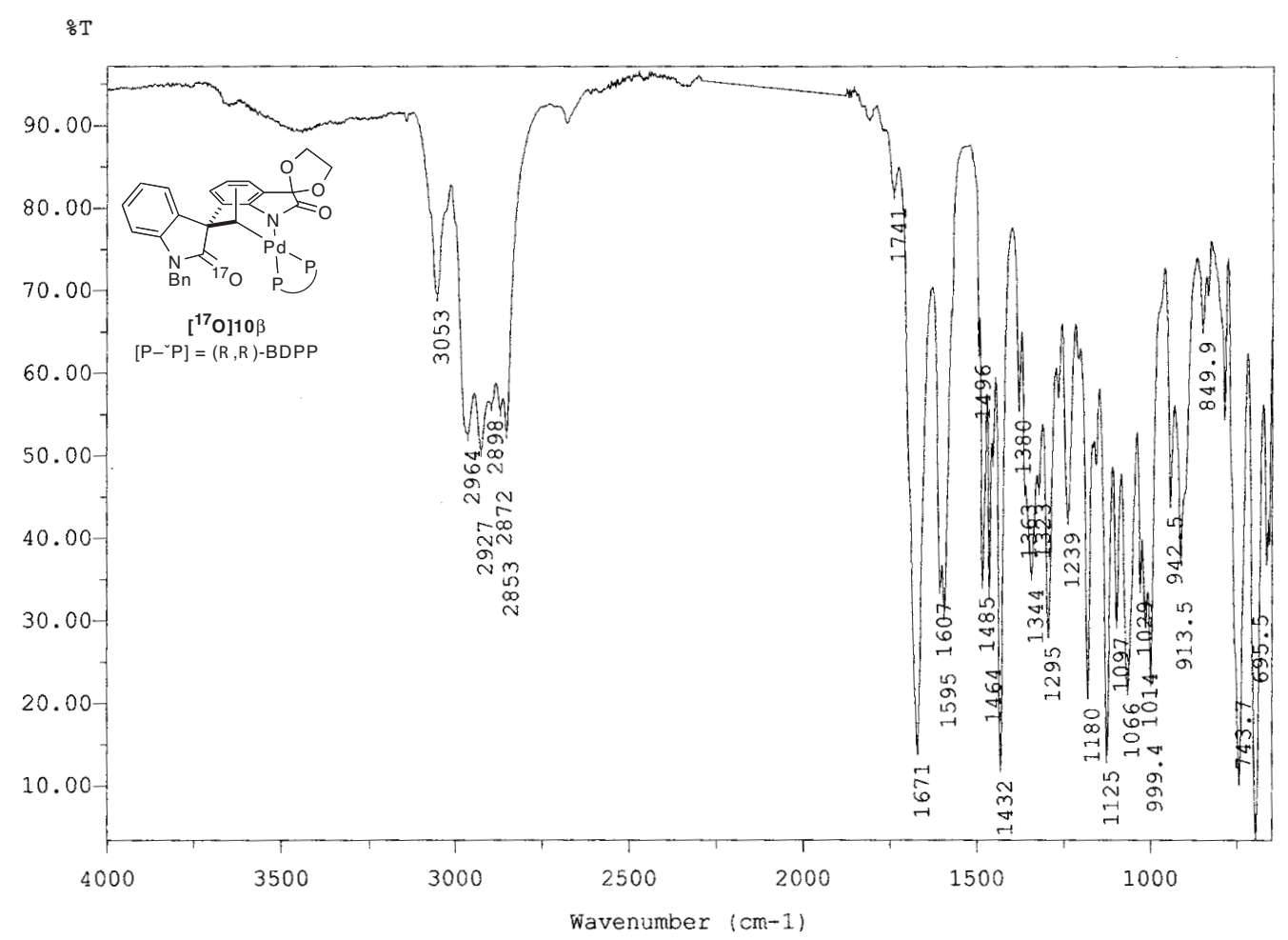

cationic 170 BDEP palladacycle high res

bjb-v- 41

$08 / 6 / 2004$

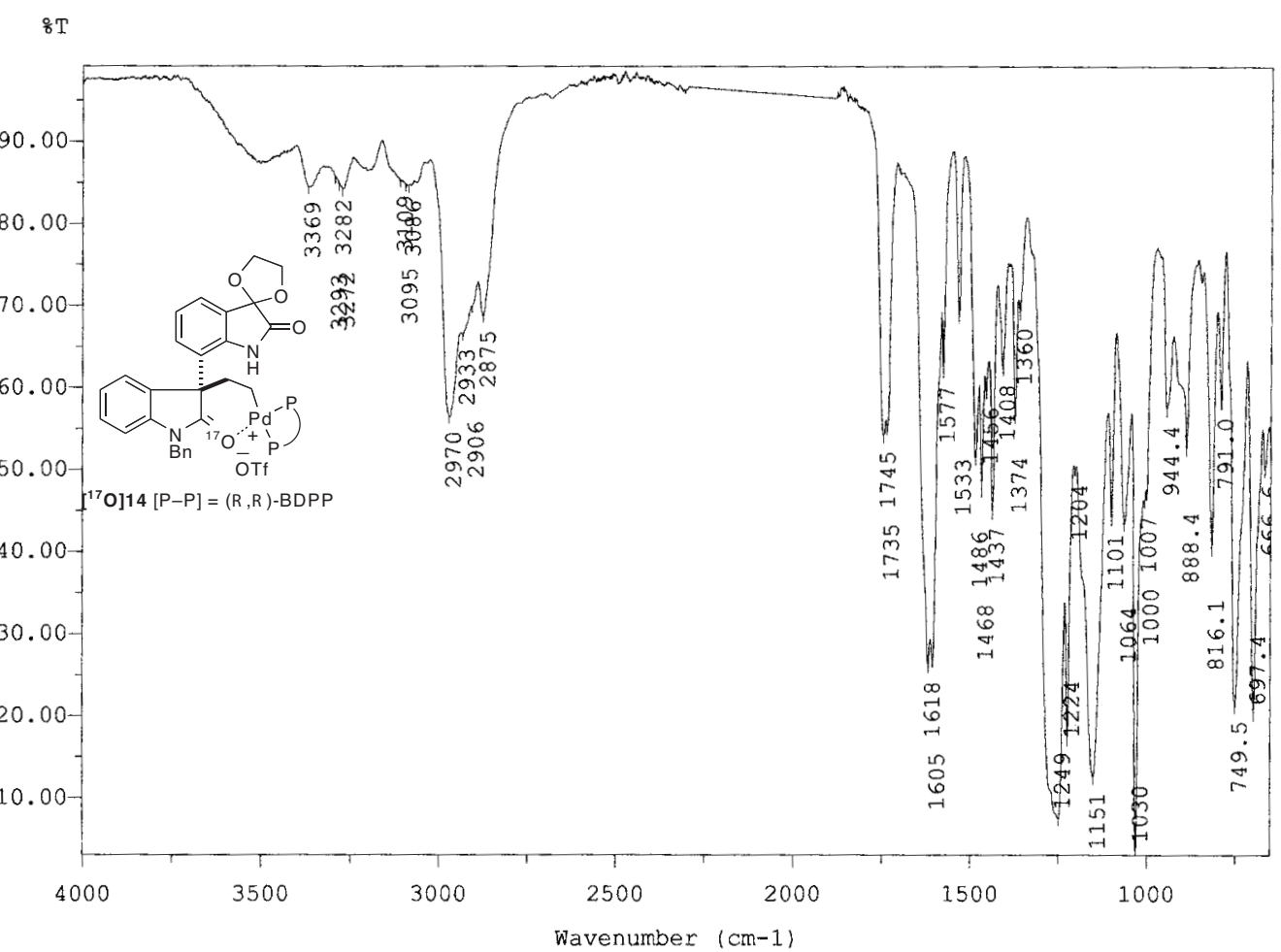

